\title{
Medium Access Control Optimization For Structural Health Monitoring Using Wireless Sensor Network
}

\author{
by \\ Saurabh Singh
}

A thesis

submitted to the Victoria University of Wellington in fulfilment of the requirements for the degree of

Master of Engineering in Network Engineering.

Victoria University of Wellington

2015 



\begin{abstract}
Wireless sensor networks (WSNs) are designed for sensing phenomena and acquiring data. In structural health monitoring (SHM) of engineering structures, increasingly large number of sensor nodes are deployed to acquire data at the spatial density, needed for structural integrity assessment.

During catastrophic events like earthquake there is a surge in simultaneous production and transmission of data to a central server at remote location. The increased contention for the wireless channel increases the probability of packet collisions resulting in packet drops, multiple transmission attempts and consequent delays. It is also not uncommon to find certain nodes (e.g. closer to sink) having better success rate in transmission of data and thereby leading to biased data delivery. Many solutions to the problem exist and clustering is the most commonly used method among then, wherein sensor nodes are grouped together. While the existing clustering algorithms do solve the network contention problems, the problem of cluster bias induced due to the proximity to sink node still remains to be addressed. Moreover all the existing solutions are very much node centric.

This thesis presents a new perspective on cluster based WSNs designed to tackle Medium Access Control (MAC) layer congestion associated with burst packet generation in an unbiased manner, thereby making it more efficient for applications like SHM. In addition to solving the network bias problem, the proposed design also ensures faster transmission times, increased throughput and energy efficiency.
\end{abstract}




\section{Acknowledgments}

First and foremost I would like to express my deepest gratitude to my supervisor Prof. Winston Seah, for his constant, consistent and invaluable guidance, without which this work would not have been possible. He immensely inspired, motivated and encouraged me every time I attended his meetings. He has always been a beacon whenever I went adrift.

I am also grateful for the help and guidance of Dr. Bryan $\mathrm{Ng}$., financial and infrastructure support of Victoria University of Wellington, my family and friends. 


\section{Contents}

1 Introduction 1

1.1 Research Problems . . . . . . . . . . . . . . . 6

1.2 Objectives ..................... 6

1.3 Contributions .................. 7

1.4 Thesis Organization . . . . . . . . . . . . . . 7

2 IEEE802.15.4 Background 9

2.1 Introduction . . . . . . . . . . . . . . . 9

2.2 MAC Sublayer Functional Overview . . . . . . . . . . . . . 11

2.2.1 Beacon Mode . . . . . . . . . . . . . . . . 12

2.2.2 Non-Beacon Mode . . . . . . . . . . . . . . . . . . 15

2.3 Data Reliability . . . . . . . . . . . . . . . . 15

3 Related Work $\quad 17$

3.1 It Is All About The Data . . . . . . . . . . . . . . . . . . 17

3.2 It Is All About The Network . . . . . . . . . . . . . . . . . . 21

3.3 Summary ....................... 24

4 Design $\quad 25$

4.1 Design Strategy . . . . . . . . . . . . . . . . . . 25

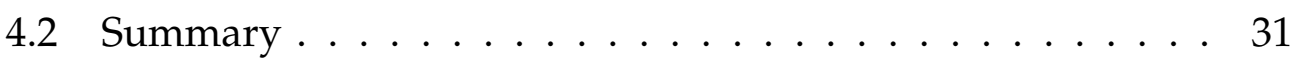

5 Evaluation $\quad 33$

5.1 Evaluation Model . . . . . . . . . . . . . . . . . 33 
5.2 Result Evaluation . . . . . . . . . . . . . . . . . . 35

5.2.1 Cluster-Centric Result Evaluation . . . . . . . . . . 35

5.2.1.1 Comparing Fairness . . . . . . . . . . . . . 35

5.2.1.2 Comparing Speed . . . . . . . . . . . . . 41

5.2.2 Node/Network Wide Result Evaluation . . . . . . . . 46

5.2.2.1 Comparing The Averages . . . . . . . . . . . 46

5.2.2.2 Comparing The Throughput and Goodput . 50

5.2.2.3 Comparing Delay . . . . . . . . . . . 52

5.2.2.4 Comparing Performance In Lossy Environment . . . . . . . . . . . 55

5.2.2.5 Comparing The Energy Consumption . . . 59

5.2 .3 Optimal Cluster Size . . . . . . . . . . . . . . . . 61

5.3 Summary . . . . . . . . . . . . . . . . . . . . . . . 69

6 Conclusion 71

$\begin{array}{ll}\text { A Energy Consumption Calculation } & 75\end{array}$ 


\section{List of Figures}

1.1 Wireless Sensor Node (picture from spie.org) . . . . . . . . . 1

1.2 Traditional Structural Sensing . . . . . . . . . . . . . 2

1.3 SHM of Bridge using WSN (picture from structural health monitoring in fujitsu.com) ............ 3

1.4 Hierarchical Network Topology . . . . . . . . . . . . . . 4

2.1 Cluster Tree Network (Adapted from [22]) . . . . . . . . . . . 10

2.2 IEEE802.15.4 Network Topologies (Adapted from [22]) . . . 11

2.3 Superframe without GTS (Adapted from [22]) . . . . . . . . 13

2.4 Superframe with CAP and CFP (Adapted from [22]) . . . . . 13

2.5 Communication Diagram (Adapted from [22]) . . . . . . . . 14

2.6 Unslotted CSMA/CA Algorithm (Adapted from [22]) . . . . 16

4.1 Deployment Scenario (figure adapted from picture on wireless building automation in wlba.wordpress.com) . . . . . 26

4.2 Node Organization . . . . . . . . . . . . . . . . 27

4.3 State Transition Diagrams . . . . . . . . . . . . . . . . . . 29

5.1 Example Qualnet Deployment Snapshot $(\mathrm{N}=250)$. . . . . . 34

5.2 Comparing Fairness $(\mathrm{N}=100, \mathrm{C}=10) \ldots \ldots . \ldots . \ldots 36$

5.3 Comparing Fairness $(\mathrm{N}=150, \mathrm{C}=15) \ldots \ldots \ldots . \ldots . \ldots 37$

5.4 Comparing Fairness $(\mathrm{N}=200, \mathrm{C}=20) \ldots \ldots . \ldots . \ldots 38$

5.5 Comparing Fairness $(\mathrm{N}=250, \mathrm{C}=25) \ldots \ldots . \ldots . . \ldots 39$

5.6 Comparing Speed $(\mathrm{N}=100, \mathrm{C}=10) \ldots \ldots . \ldots . \ldots 42$ 
5.7 Comparing Speed $(\mathrm{N}=150, \mathrm{C}=15) \ldots \ldots \ldots \ldots$

5.8 Comparing Speed $(\mathrm{N}=200, \mathrm{C}=20) \ldots \ldots \ldots \ldots . \ldots . \ldots . \ldots 44$

5.9 Comparing Speed $(\mathrm{N}=250, \mathrm{C}=25) \ldots \ldots \ldots$

5.10 Average Performance For Single Data Packet Per Node . . . 47

5.11 Average Performance For Multiple Data Packets Per Node . 49

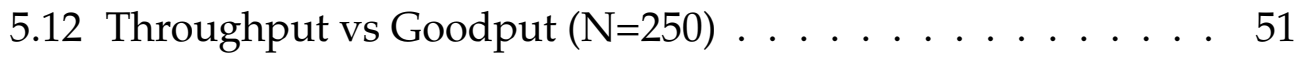

5.13 Delay Comparison $(\mathrm{N}=250) \ldots \ldots . \ldots . . \ldots 53$

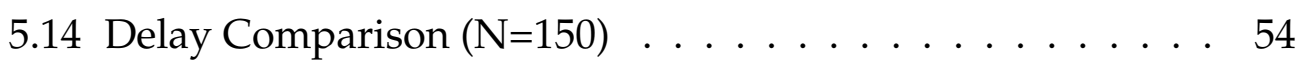

5.15 Lossy Environment with Single Data Packet Per Node( $\mathrm{N}=250$, $\mathrm{C}=10) \ldots \ldots \ldots \ldots \ldots \ldots \ldots$

5.16 Lossy Environment with Multiple Data Packets Per Node(N=250, $\mathrm{C}=10) \ldots \ldots \ldots \ldots \ldots \ldots$

5.17 Lossy Network Performance Overlap $(\mathrm{N}=150)$. . . . . . . 58

5.18 Lossy Network Performance Overlap ( $\mathrm{N}=250)$. . . . . . . . 59

5.19 Energy Consumption Comparison With WSN For SHM Proposed By Liu et al.[33] Where eT (y-axis) Is The Transmission Power, Measured In mAh. . . . . . . . . . . . . . 60

5.20 All Cluster Sizes . . . . . . . . . . . . . . . . . . . . 62

5.21 One Set Of Cluster Sizes $(\mathrm{N}=250) \ldots \ldots$. . . . . . . . . 64

5.22 All Cluster Count $(\mathrm{N}=100,150) \ldots \ldots 6$

5.23 All Cluster Count $(\mathrm{N}=200,250) \ldots \ldots$. . . . . . . . . 67

5.24 All Cluster Count $(\mathrm{N}=300) \ldots \ldots$. . . . . . . . . . 68 


\section{List of Tables}

5.1 Simulation Parameters For Cluster-Centric Comparison . . . 35

5.2 Simulation Parameters For Comparing The Averages . . . . 46

5.3 Simulation Parameters For Throughput, Goodput and Delay Comparison ................... 50

5.4 Simulation Parameters For Performance Comparison In Lossy Environment . . . . . . . . . . . . . 55

5.5 Simulation Parameters for energy consumption comparison 59

5.6 Simulation Parameters for Optimal Cluster Size . . . . . . . 61

5.7 Simulation Parameters for Unequal Cluster Size . . . . . . . 63

5.8 Simulation Parameters for Optimal Cluster Count . . . . . . 65

A.1 Values Used For Energy Consumption Calculation . . . . . . 75 


\section{Chapter 1}

\section{Introduction}

\section{WSN and SHM Background}

A wireless sensor network (WSN) is a spatially distributed network of autonomous sensor nodes. Wireless sensors nodes combine sensing, computation and wireless communication, all in one place (see Figure 1.1). Its emergence can be traced back to an initiative by the National Research Council [1]. Typically applications are battlefield surveillance [2], environmental monitoring [3], structural health monitoring(SHM) [4], etc.

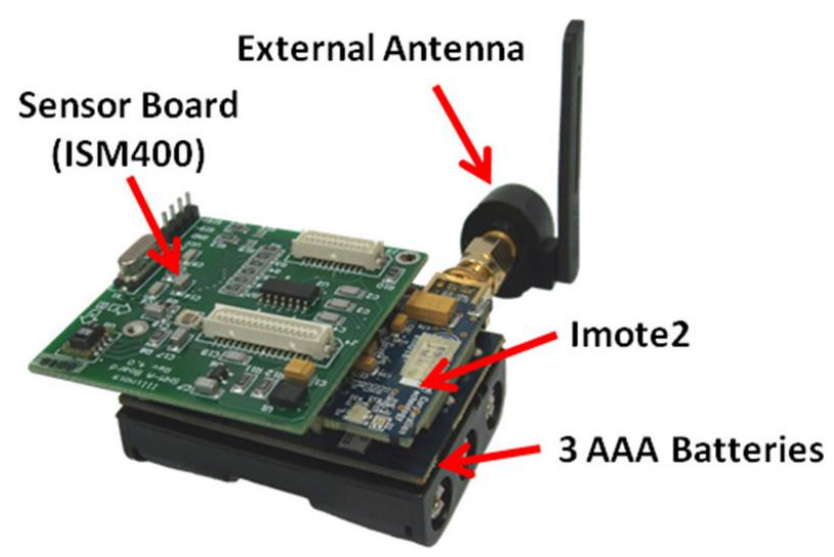

Figure 1.1: Wireless Sensor Node (picture from spie.org) 
Use of WSNs in SHM has received increased attention from the research communities in recent years. SHM [5] is the practice of monitoring health and properties of structures built by structural engineers. SHM is used for detection of gradual deterioration of critical infrastructures like buildings, bridges, and aircrafts [6].

In early days, engineers used to monitor structural health manually (Figure 1.2), but as the structures have become large and complex over the time, the task of monitoring in itself has also become more difficult. Monitoring task has now been taken over by sensor nodes embedded in structures, which can monitor and report data in nearly real time.

Structural engineers have used wired sensors [7] to collect massive amounts of data that they require for structural integrity analysis. Installing point to point connections can be very costly $[8,9]$ and time consuming. High cost factor and ease of deployment led structural engineers to replace the traditional tethered monitoring systems with WSNs in SHM (see Figure 1.3).

WSN has been widely used in SHM applications of buildings [10], bridges [11] and aircrafts [12]. Like the Tsing Ma bridge [13] in Hong Kong and the Imperial County Services Building [14] in California.

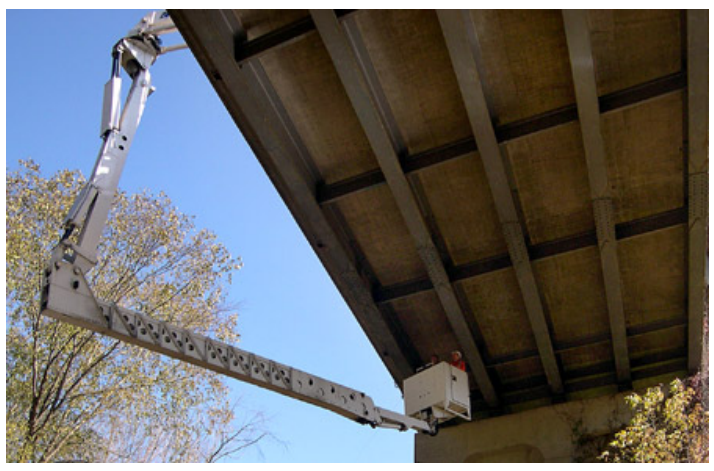

(a) Traditional Bridge Sensing (picture from northwestern.edu)

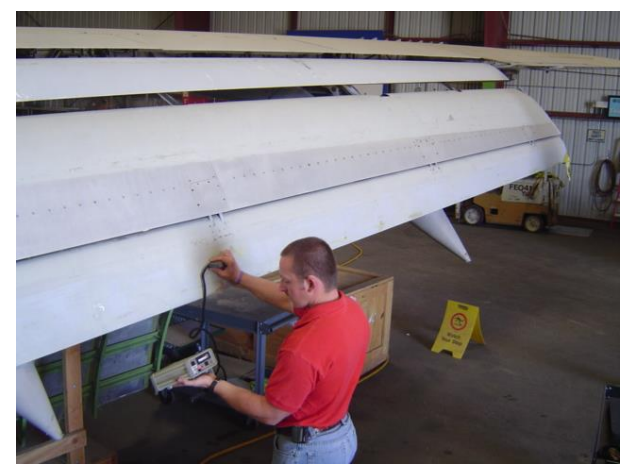

(b) Traditional Aircraft Sensing (picture from compositesworld.com)

Figure 1.2: Traditional Structural Sensing 


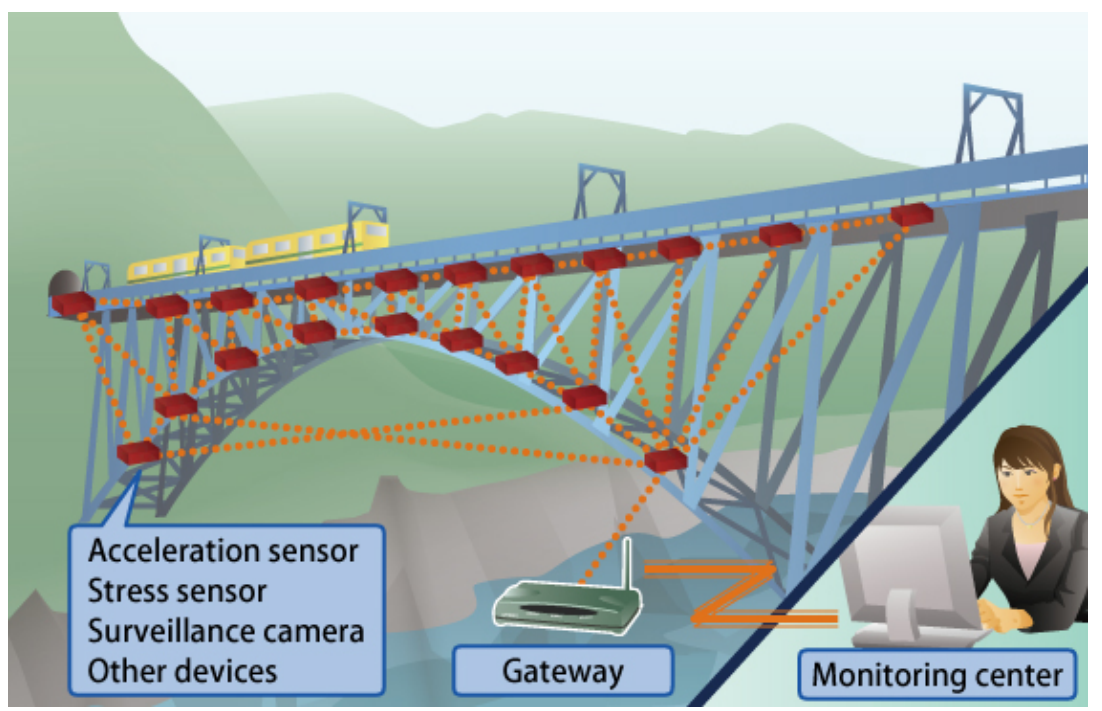

Figure 1.3: SHM of Bridge using WSN (picture from structural health monitoring in fujitsu.com)

\section{Problems With The Use of WSNs in SHM}

The low cost nature of WSNs enabled higher spatial density, which was a cause of concern with traditional tethered monitoring systems due to high costs. WSNs eliminated the need for wires to connect sensor nodes to the data acquisition centre, but it also presented a new set of problems.

Power constraint is a key design issue with WSNs [15, 16, 17] which are expected to last long periods of service. Typically WSNs are battery powered, but alternative energy sources like energy harvesting [18] are increasingly sought after.

WSNs usually relax packet delivery latency [19] and network throughput requirements in favour of reducing energy consumption, like S-MAC [20] and T-MAC [21]. For this purpose, the IEEE802.15.4 standard [22] designed for low-rate wireless personal area networks (LR-WPANs) is a natural choice for WSNs. In SHM, sensors can be scheduled to operate with low duty cycles [23] to minimize contention and save energy. 
In SHM, an event (like earthquake) can generate huge amounts of data at all nodes simultaneously, which can lead to a bursty network. WSNs are not designed to handle such network bursts [24]. Moreover, SHM applications tend to operate in environments that are harsh to wireless communication [4], e.g. presence of metal beams that interfere with radio signals which results in increased packet loss. This sudden influx of data into the network leads to severe network congestion, packet drops, delays, and repeated retransmission attempts. Early adoptions of the technology focused on raw data collection at sink nodes $[25,26,27]$ which led to such massive bursts.

Another problem prevalent with SHM is the network bias towards nodes closer to the sink node, which is a common wireless network phenomena. This is because of the presence of capture effect [28], wherein received signal strength at the sink node differs based on transmitter-receiver distance. This leads to better transmission success ratio, lesser retransmission attempts, and comparatively lower energy consumption for sensor nodes closer to the sink node, compared to the nodes farther away.

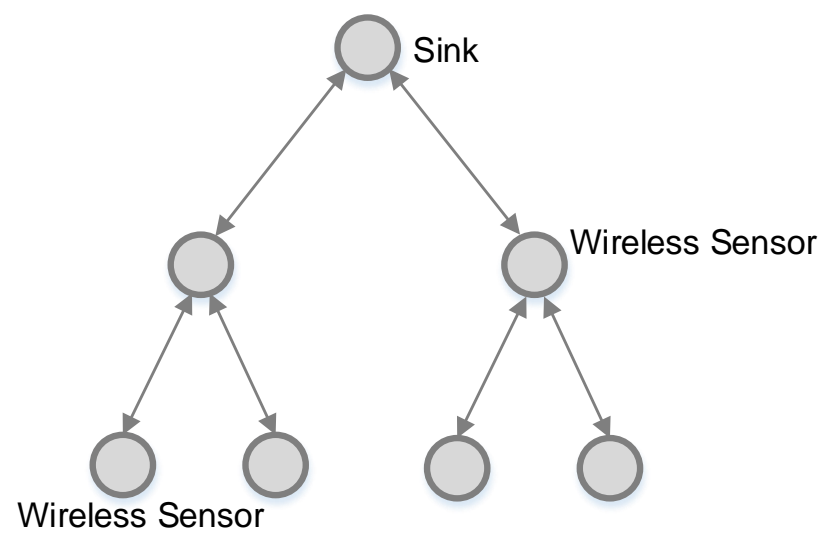

Figure 1.4: Hierarchical Network Topology 
As the size of WSN grows in response to real-world application needs, scalability also becomes an issues, commonly solved by adoption of a hierarchical network architecture (Figure 1.4) and the use of clustering [29].

Any new research regarding the use of WSNs in SHM need to consider these problems.

\section{Research Motivation}

Majority of existing clustering approaches have energy conservation as primary focus [30]. To handle the high volumes of data while providing the necessary useful information required by structural engineers, some clustering schemes resort to data aggregation/fusion or using in-network processing. The data aggregation or fusion techniques [31] employ collection of data at cluster heads and then attempt to reduce the number of duplicate or correlated data packets before transmitting the processed data. Performing in-network processing [32, 33, 34, 35] of SHM data requires in-depth domain knowledge to be integrated into the networking subsystem, which limits the use of the proposed schemes in other SHM scenarios.

This motivates the research to propose a design that retains all the critical raw sensed data by not performing data aggregation/fusion or innetwork processing, in addition to answering all the problems associated with the use of WSNs in SHM discussed above.

The proposed MAC protocol prototype also exploits the clustering characteristic in its design. Sensors nodes that generate highly correlated data are grouped into a cluster. In practice however, the clusters are defined by domain experts, e.g. structural engineers.

The proposed MAC protocol arbitrates access to the wireless channel such that every cluster has a fair opportunity to transmit data. This is achieved by viewing each cluster as a supernode. This way, reduction of contention, and fast and fair transfer of data is achieved. Consequently, the proposed approach is designed to provide structural engineers the (raw) 
data that they need and at the same time address WSN constraints.

\subsection{Research Problems}

This thesis deals with the following problems that can be identified in bursty wireless networks:

- Increased network bias towards nodes closer to the sink node.

- Increased transmission delay due to high network congestion.

- Increased retransmission attempts due to high packet loss.

- Increased usage of energy due to high retransmission attempts.

- Decreased network goodput due to increased network throughput, resulting from high retransmission attempts and packet loss.

- Reduced scalability of bursty networks with increased network size.

\subsection{Objectives}

This thesis focuses on MAC protocol optimization in SHM, so the key objectives of the proposed design are as under:

- Reduce network bias by fairly allocating channel to sensor nodes.

- Reduce transmission delay, and thereby reduce the average overall successful network data transmission time.

- Reduce the number of retransmission attempts, which should also lead to reduced energy consumption.

- Increase the network goodput by reducing the packet losses and thereby increasing the successful data transmissions. 
- Maintain scalable network performance even with increased network size.

\subsection{Contributions}

This thesis proposes a cluster-centric MAC protocol that answers all the above mentioned research problems and meets the stated objectives. The proposed design achieves the following key improvements over the standard IEEE802.15.4 protocol:

- Network bias is eliminated and all the nodes get fair opportunity to transmit their data packet.

- Transmission delay is significantly reduced, and network data packets are transmitted faster to the sink node.

- Retransmission attempts are significantly reduced, which also leads to reduced energy consumption.

- Network congestion is reduced, which results in decreased packet loss and improved goodput.

- Network scalability is maintained with increasing network size by delivering consistently better performance compared to other algorithms.

The proposed research findings and evaluation have been accepted by the WiOpt conference [36].

\subsection{Thesis Organization}

The thesis is organized as follows:

- Chapter 2 provides a brief background of the standard IEEE 802.15.4 protocol needed for the understanding of related work and proposed design. 
- Chapter 3 presents an overview of related work in use of WSN in SHM.

- Chapter 4 discusses the design and implementation of the proposed cluster-centric MAC.

- Chapter 5 evaluates the proposed design against existing protocols and also presents a new way to analyse network performance from cluster-centric network perspective.

- Chapter 6 contains the conclusion and suggestions for further research in the field. 


\section{Chapter 2}

\section{IEEE802.15.4 Background}

The base protocol for use in WSNs is defined by the IEEE802.15.4 [22] protocol standard. It mainly defines the physical and MAC layer specifications. This chapter gives a brief overview of the MAC layer specification; defined by the IEEE802.15.4 standard; relevant for understanding of the related work and the proposed design.

\subsection{Introduction}

The IEEE802.15.4 protocol is designed for low-rate wireless personal area networks (LR-WPAN). An LR-WPAN is a simple, smaller in scale, and cost effective network designed to work for applications with relaxed throughput and power requirements. An ideal LR-WPAN should meet the following requirements:

- Simple installation/deployment.

- Low cost.

- Lower power consumption, hence long battery life.

- Short range operation. 
- Data reliability.

This protocol mainly consists of two types of devices, namely coordinators and others simply called devices. A personal area network (PAN) typically has multiple devices and one coordinator, however a PAN coordinator may also be used to join two neighbour PANs to make a larger cluster tree network as shown in Figure 2.1. Depending on application requirements, an IEEE802.15.4 network may be configured as either a star topology (Figure 2.2a) or a peer-to-peer topology (Figure 2.2b).

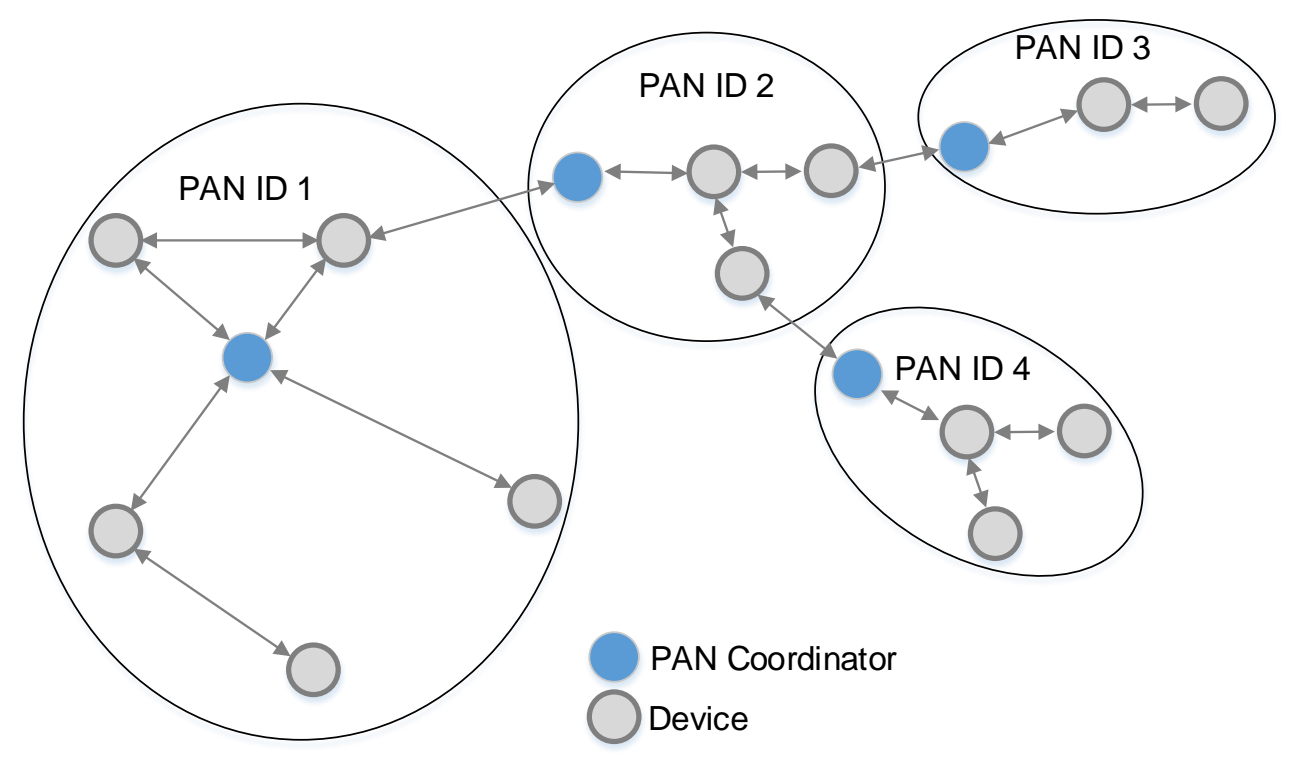

Figure 2.1: Cluster Tree Network (Adapted from [22])

Based on functionality these devices can be classified as:

1. Full Function Device (FFD) - These devices can function as a personal area network (PAN) coordinator, a coordinator, or a simple device. An FFD can talk to all the devices in the PAN, which makes it suitable for use in both peer-to-peer topology and star topology. FFDs can thus be used for routing as well. 


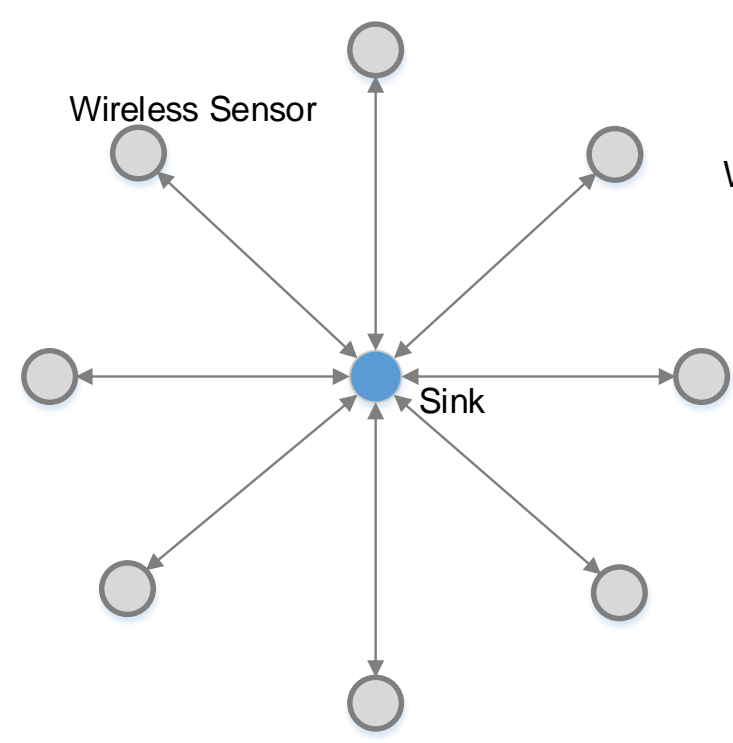

(a) Star Topology

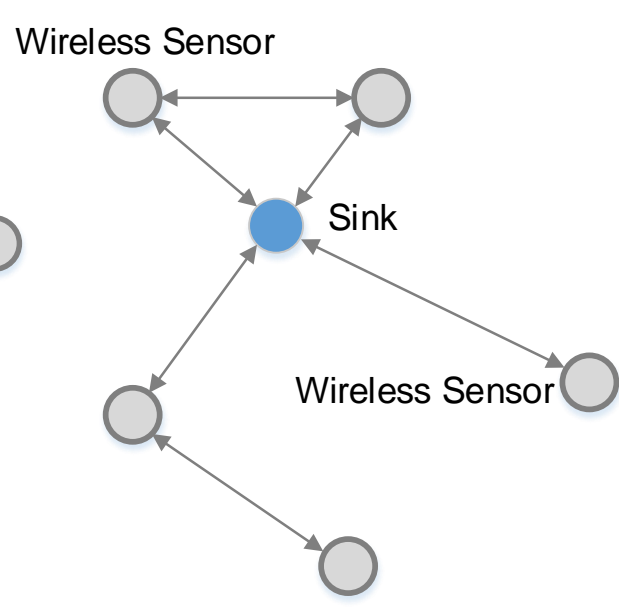

(b) Peer To Peer Topology

Figure 2.2: IEEE802.15.4 Network Topologies (Adapted from [22])

2. Reduced Function Device (RFD) - These devices function only as simple devices and cannot perform any coordination function. Since these are not supposed to handle large amounts of data, these have comparatively limited resources and memory capacity. RFDs can associate with only a single FFD at a time, which makes them ideal for use only in star topology.

\subsection{MAC Sublayer Functional Overview}

The MAC sub-layer of the IEEE802.15.4 protocol mainly oversees beacon management, guaranteed time slot (GTS) management, channel access, MAC frame acknowledgement, device association and dissociation.

The IEEE802.15.4 protocol specifies two different modes for transmitting data to the PAN coordinator which are described as follows: 


\subsubsection{Beacon Mode}

The beacon mode uses regular interval beacons to synchronize the network and control transmission cycles. Each interval between consecutive beacons consists of a superframe, the format of which is defined by the coordinator. A superframe starts with a beacon and is divided into 16 equal sized slots. The IEEE802.15.4 defines two types of superframes:

1. Superframe without GTS - The 16 slots of superframe are divided into 1 slot of beacon and 15 slots of contention access period (CAP) (Figure 2.3a), wherein devices use slotted CSMA/CA mechanism to access channel and transmit. Slotted CSMA/CA is CSMA/CA aligned with the slot boundaries of superframe. All the transmissions have to complete by the end of the last transmission slot on the CAP. Optionally the CAP maybe followed by an inactive period (Figure 2.3b), wherein the coordinator may enter low-power mode to conserve energy.

2. Superframe with GTS - The 16 slots of superframe are divided into 1 slot of beacon, and remaining slots are taken up by the contention access period and contention free period (CFP) (Figure 2.4). CAP are always immediately followed by CFP. The PAN coordinator can allocate up to 7 GTS, and a GTS may extend more than one time slot. All the GTSs together make up the CFP. All GTS transmissions have to complete within the allocated slot boundaries.

An example communication diagram for data transmission to coordinator in a beacon enabled PAN is shown in Figure 2.5a. 


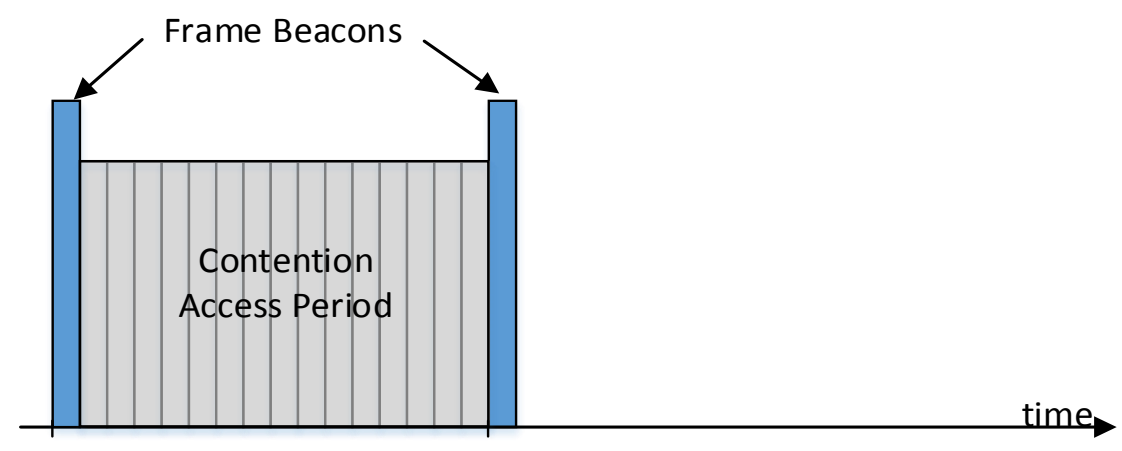

(a) Superframe with CAP

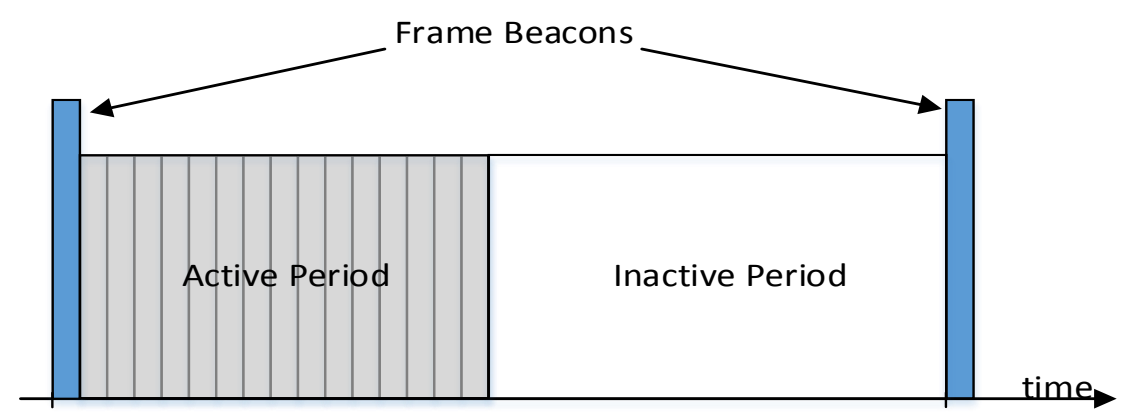

(b) Superframe with Active and Inactive Period

Figure 2.3: Superframe without GTS (Adapted from [22])

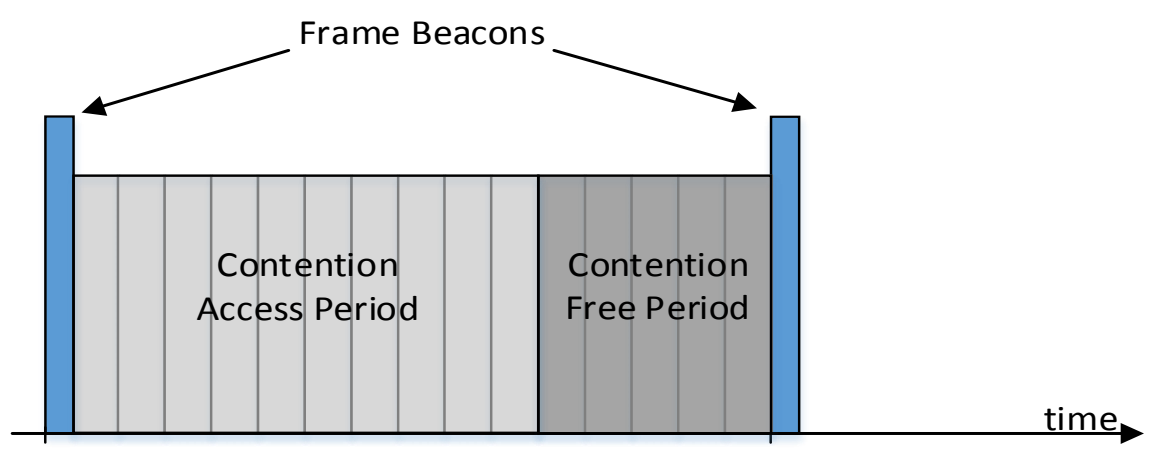

Figure 2.4: Superframe with CAP and CFP (Adapted from [22]) 


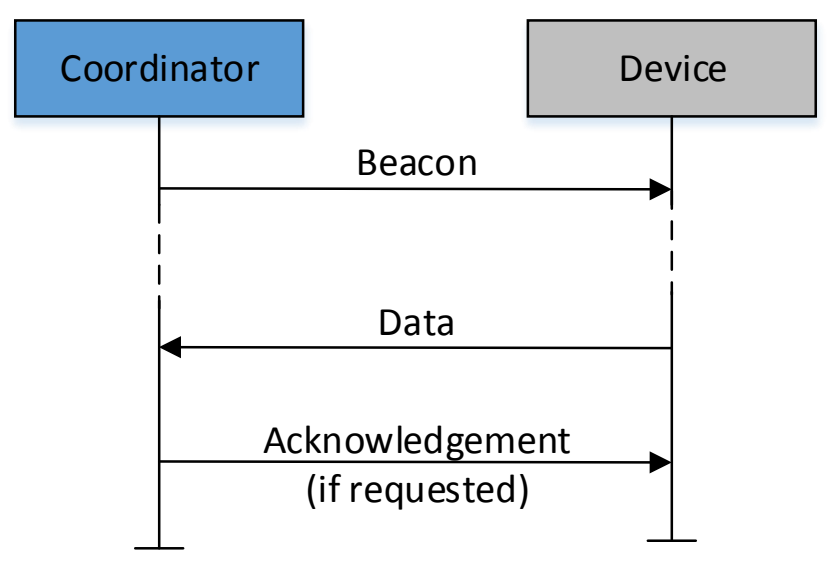

(a) Beacon Enabled PAN

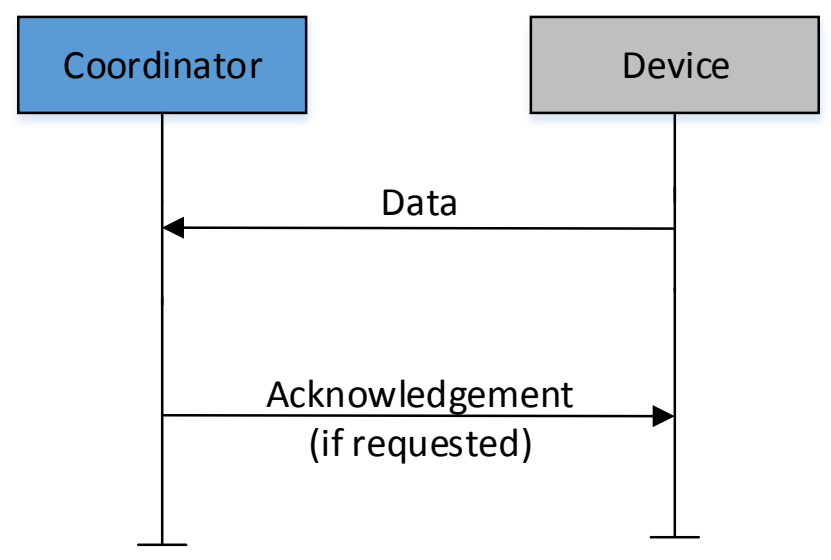

(b) Non-Beacon Enabled PAN

Figure 2.5: Communication Diagram (Adapted from [22]) 


\subsubsection{Non-Beacon Mode}

The non-beacon mode uses simple unslotted CSMA/CA to arbitrate channel access. When a device wants to transfer its data to the PAN coordinator, it simply uses the standard unslotted CSMA/CA mechanism to access channel and transmit data, which maybe followed by an optional acknowledgement frame. The complete transaction sequence is shown in Figure 2.5b.

This mode of transfer requires little configuration and is highly scalable, which makes it much preferable for use in WSNs.

\subsection{Data Reliability}

To improve the probability of successful transmission IEEE802.15.4 uses frame acknowledgements, 16-bit frame checksum, and the CSMA/CA mechanism. Since frame acknowledgement and frame checksum are beyond the scope of this thesis, only the CSMA/CA mechanism shall be discussed.

The IEEE802.15.4 protocol defines two different types of CSMA/CA mechanisms, that are slotted and unslotted CSMA/CA. Slotted CSMA/CA is used only when the superframe is in use, which happens only in the beacon mode. Slotted CSMA/CA and unslotted CSMA/CA function similarly except a few key differences:

1. Backoff Boundary - Throughout the backoff procedure slotted CSMA/CA keeps itself aligned to the slot boundaries. Right before the backoff it first aligns to the slot boundary then delays for a random number of transmission slots. Unslotted CSMA/CA on the contrary never aligns itself to any transmission slot and performs backoff for a random amount of time.

2. Contention Window (CW) - Slotted CSMA/CA uses CW to take transmission decision when the channel is found idle post backoff. $\mathrm{CW}$ is set to a default value of 2 before backoff, and decremented 
by 1 every time the channel is found idle post backoff. When CW reaches zero, the data is transmitted. On the contrary; in unslotted CSMA/CA, the data is transmitted immediately after the backoff if the channel is found idle, which works similar to a $\mathrm{CW}$ of size 1.

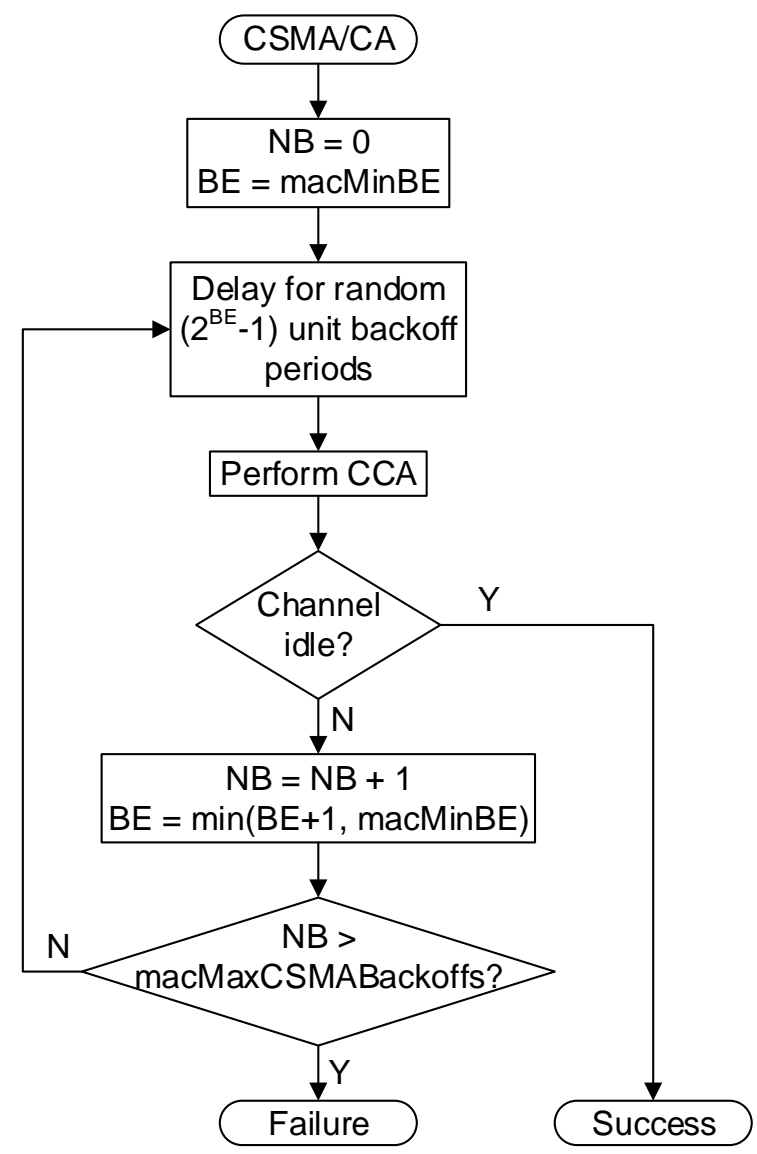

Figure 2.6: Unslotted CSMA/CA Algorithm (Adapted from [22])

The proposed design uses a modified version of the standard unslotted CSMA/CA algorithm as shown in Figure 2.6, which shall be discussed in detail in Chapter 4.

The performance evaluation of the standard IEEE802.15.4 MAC protocol is done in Chapter 5. 


\section{Chapter 3}

\section{Related Work}

This chapter presents the related work done in the field of SHM with WSN. The work has been broadly classified into two categories:

1. Motivated by information (generated by events) constraints.

2. Motivated by network constraints in SHM applications.

\subsection{It Is All About The Data}

Straser et al. [37] were the first to give a proof of concept of sensor network for use in SHM. Their solution was to simply transmit raw sensor data right after occurrence of a seismic event and perform modal analysis for damage identification at server end. This acted as a big stepping stone for future generation of WSNs for use in SHM. Such WSN based SHM systems collected raw sensor data without special consideration to bandwidth and energy constraints, similar to traditional wired systems.

Structural health assessment of huge structures require massive amounts of data $[38,39]$. This came about from the use of modal/frequency parameter estimation techniques that require large amounts of data collected from a dense array of sensors $[40,41]$. There are essentially two types 
of damage detection techniques, frequency based damage detection and modal shape based damage detection.

Frequency based damage detection methods [42] require huge computations, and damage model assumptions. These methods requires a priori knowledge of the structure before damage identification can be performed. Modal shape based damage detection have been proven to be much accurate and faster compared to the frequency based damage detection techniques [43]. Modal shape analysis however requires response time history of the structural vibrations for structural health assessment [44].

Transmitting such huge amount of raw sensed data can lead to a bursty network. In WSNs data transmission is the primary energy hogger [45] and in burst networks it can lead to significant consumptio of energy. The data produced by SHM systems is highly correlated in the space domain [46], which is a characteristic exploited by various node clustering algorithms (e.g. [32, 33, 34, 35]). This motivated research on distributed damage estimation techniques.

Caffrey et al. [47] proposed that instead of transmitting raw sensor data, Fourier spectra of the structural vibration time history can be calculated within the nodes. The resultant modal frequency can thus be transmitted to the sink node, thereby conserving bandwidth, raw data transmission overhead and battery. The central repository can then perform modal frequency analysis to detect damage. Even though the proposed model performed centralized damage estimation, it proved that processed sensor data is sufficient enough to detect structural damage.

Another approach for distributed damage estimation technique is by using distributed modal-shape analysis. A simple cluster-based approach was proposed by Zimmerman et al. [32] using pre-defined two-node clusters where all nodes are within communication range of the sink node. The study adopted output-only modal identification [40] to monitor the response characteristics of exciting a large civil structure in a controlled manner. The goal of the study was to demonstrate that distributed estimation 
techniques can be embedded within the wireless sensors to mitigate the data deluge. The limited scope of the study did not consider other topologies nor how to optimize the clustering to reduce energy consumption of WSNs.

This motivated Liu et al. [33] to study how to optimally partition sensor nodes such that requirements from both modal analysis and WSNs (with regard to energy efficiency) are addressed. In their approach, the whole network is divided into single-hop clusters, each with a cluster head $(\mathrm{CH})$ that performs analysis. They show that performing modal analysis at $\mathrm{CHs}$ and transmitting the processed bits takes up less energy, as compared to the traditional approach of sending all packets to the sink, even when the whole network is configured as a shortest path tree.

The work by Zimmerman et al. [32] was used by Jindal and Liu [34] to construct an optimal data forwarding and computing structure that minimizes energy consumption subject to a computational delay constraint. This study extends the earlier works by providing an efficient routing structure that is designed for distributed computation of SHM algorithms. Like [33], the algorithms were validated with simulations and testbed experimentation. Most of the simulation results were based on 30 node topologies with node density of 8 nodes per $m^{2}$. For the experimental validation, 12 nodes were used as compared to the 10 node setup adopted in [33], which is a very low node density compared to a real-life SHM network.

To further reduce the data dimensionality (and energy consumption), Hackmann et al. [35] proposed a flexible multi-level monitoring approach that incrementally activates sensors in damaged regions on demand, keeping most of the sensors asleep until they are needed. Under normal routine conditions, also known as first stage, a small number of sensors spread across the structure being monitored are enabled as a single cluster to perform damage identification. If no damage is detected, these sensors return to sleep. If damage is detected, additional sensors in the vicinity of the damage (i.e. detected by the first stage sensors) are activated to narrow 
down the region of damage. This process is repeated until the desired resolution for the damage location is achieved. Different sensors can be activated during different stages, as well as during the same stage at different times. This enables load balancing among the sensors to extend the lifetime of network as a whole.

Lynch et al. [48, 49] and Liu et al. [33] have proven through comparative study that in-network processing of data is more power efficient compared to raw time-history data transmission.

Various data compression techniques to reduce bandwidth consumption of raw sensor data have also been proposed. Salomon and David [50] have used lossless Huffman coding to encode raw data. Park et al. [51] propose principal component analysis based compression technique. Sazonov et al. [52] propose on-the-fly compression technique.

Another way to reduce bandwidth consumption is by using data aggregation techniques $[53,29,31,54]$ that aim to remove redundant data and improve bandwidth utilization. Most of these techniques are cluster based. The key functions of clustering in WSNs are $\mathrm{CH}$ selection [55], cluster formation [56] and data transmission, all of which are concerned with the efficient operation of the network with little consideration of the application data requirements.

In the WSN approaches motivated by data constraints, a priori knowledge of the structure needs to be pre-computed. Also it cannot adapt to changing network [57] (e.g. fluctuating wireless link quality) or structural (modal frequency shift due to environmental effects) conditions, nor deal with network traffic dynamics. Another potential problem is that losing a single packet of processed data may render all information useless. WSN approaches motivated by network constraints [30], on the other hand, have been designed to address fluctuating network conditions with energy conservation as the primary aim while maintaining network connectivity but without explicit consideration for the quality of the data content. 


\subsection{It Is All About The Network}

The IEEE802.15.4 MAC protocol is based on the standard CSMA/CA. It is simple and works well for low-rate and low-power networks making it the natural choice of transmission method for WSNs. While this protocol works for light traffic (i.e. low-rate) its performance quickly degrades when network traffic increases, especially in the presence of bursty traffic, due to large scale network collisions [24, 58, 59].

Due to limited control over transmission, from its decentralised nature, the performance of CSMA/CA can be highly unpredictable. There can also be bias towards certain nodes over others, depending on their distance from the PAN coordinator. The natural approach to deal with these constraints is to exploit the inherent correlation in SHM data and performing in-network processing that results in much smaller processed data, that can fit within the bandwidth and energy constraints, as previously discussed. The alternative would be to modify the IEEE802.15.4 protocol to support heavier traffic loads that deal with bursty traffic.

Nefji and Song [60] proposed CoSenS, a collect-and-send burst scheme in which they try to improve the intrinsic performance of CSMA/CA in WSNs. CoSenS is implemented on top of the CSMA/CA protocol, where it collects data from the (children) sensors and neighbouring routers for a period of time, referred to as the Waiting Period (WP). At the end of WP, the router transmits all the collected data packets in a burst during a period referred to as the Transmission Period (TP).

The performance improvements in terms of throughput, end-to-end delay and reliability have been validated using simulations, and the authors claimed that they are the first to improve other aspects of WSN performance, unlike other WSN MAC schemes which only aimed to improve energy efficiency. While throughput and reliability improvement are obvious, the delay improvement can be inferred from few collisions among network nodes, viz. routers, as compared to individual packet transmis- 
sions. CoSenS can be regarded as a subset of the WSN approaches for SHM discussed earlier, without in-network processing or consideration for the intrinsic correlation of SHM data. The contention among the sensors transmitting to the PAN coordinator (in their case, the router) remains unaddressed.

Closer to addressing the contention issue in IEEE802.15.4-based WSNs is the scheme by Lee et al. [61] which aims to ensure balanced distribution of data transmission across groups/clusters of nodes in a network. When the successful data transmission ratio (SDTR) of the network falls below a lower threshold the proposed algorithm kicks in, and continues to operate until SDTR exceeds another (higher) threshold. The scheme operates by dividing the network into groups or clusters of nodes. The original IEEE802.15.4 superframe is modified such that the Contention Access Period (CAP) is divided into Group CAP (G-CAP) and Free CAP (F-CAP) where G-CAP comprises multiple Guaranteed Time Slots (GTS) one for each group.

After the allocation of a GTS, the nodes within the group send simultaneous pulses to the coordinator if they have data to send. If the coordinator receives no pulse, that means that the group has no data to send; if the coordinator receives one pulse, it means that the group has just one data packet to send, and if the coordinator receives a garbled pulse then the group has more than one data packet to send. Future allocation of GTS depends on the number of pulses in the current round.

This novel scheme of pulses is very effective for a small network, but does not scale to large network/group sizes, as nodes would spend most of their time sending pulses before actually being able to get a transmission opportunity, and thereby wasting significant time and energy. Burst traffic scenarios are also not addressed.

Research on WSNs for SHM of civil infrastructures after catastrophic rare events has explored the use of energy harvesting to drive the sensors. Given the very limited amount of energy that can be harvested from the event, Cheng et al. [62] proposed a modification to the IEEE802.15.4 pro- 
tocol that gives higher preference to uncorrelated data rather than individual node data. The scheme ensures that at least one data packet is transmitted from each cluster at the earliest, where each cluster represents a region of interest to structural engineers. The key consideration in their design is to minimize transmission of highly correlated data. This thesis however proposes a design that aims to achieve $100 \%$ data transmission, instead of selective data transmission.

Cheng et al. [62] analytically derive an optimal backoff time slot selection algorithm that aims to minimize the number of nodes selecting the same backoff slot, unlike the IEEE802.15.4 protocol whose random backoff slot selection follows a uniform distribution. This approach is critical for the stringent energy harvesting scenario considered by the authors as collisions waste energy and should be avoided at all costs. The proposed design also adopts the same non-uniform random backoff slot selection approach, to be presented in the next chapter.

A common pattern among majority of clustering approaches is the presence of CHs. Such approaches mainly target data aggregation, network collision reduction, and energy conservation. Problem much prevalent with these approaches is the severe stress and energy drain that data forwarding puts on the CHs. Load balancing algorithms are a common solution to such problems.

One of the first attempts at clustering of nodes was by LEACH [31], which proposed a load balancing energy efficient design. Problem with LEACH and other LEACH inspired designs [63] is the energy drain the data forwarding node would go through in bursty networks.

Much work has been done on energy-efficient selection of $\mathrm{CHs}[64,65]$, however little work has been done on energy-efficient functioning of the $\mathrm{CHs}$ themselves. This motivated Shigei et al. [66] to propose Cluster Communication based on Neighbour nodes (CCN). [66] adjusted the broadcast range of the $\mathrm{CHs}$ based on the number of neighbour nodes to reduce energy consumption, and proved that it was more energy efficient compared 
to LEACH.

Besides these probabilistic clustering algorithms, some deterministic approaches have also been proposed. Like spatial correlation based clustering $[67,68]$, centralized clustering $[69,70]$, and even preset clustering algorithms [71, 72]. All these clustering approaches aim to prolong the network lifetime, however it is imperative that the scope of clustering be expanded and other network problems be answered, like network bias towards certain nodes based on their proximity to the sink node.

\subsection{Summary}

The related work shows that the inherent problems with the use of WSN in SHM are bandwidth limitations and energy consumption. Many solutions have been proposed for this, for example, in-network processing, data aggregation, etc., but none focus on giving equal priority to all the nodes across network for channel allocation.

Bandwidth allocation bias towards nodes closer to the PAN coordinator than further away is another problem that is not an uncommon phenomena. This can lead to severe network stress and battery strain for further away nodes. This thesis approaches the SHM requirements by first answering the network bias and giving equal priority to all nodes across network. The next chapter discusses the proposed design to solve these problems. 


\section{Chapter 4}

\section{Design}

The primary aim of this thesis is to reduce congestion in bursty networks and remove network bias resulting from proximity to the sink node, which lays the base and motivation for the proposed design. This chapter presents the proposed cluster-centric design to handle bursty traffic in WSNs in an unbiased manner. The proposed design is implemented at MAC layer, with a slight modification to the standard 802.15.4 MAC protocol.

\subsection{Design Strategy}

A typical SHM system has the sink node outside the building with all the sensor nodes installed inside the building, as shown in Figure. 4.1. The proposed design assumes that the sensor nodes are just one hop away from the sink nodes. In the proposed design, the sink node acts as the PAN coordinator and has to be an FFD, while the sensor devices can be either FFD or RFD since the network is designed as star topology.

The clusters of sensors are defined by domain experts, e.g. structural engineers, who have the knowledge to best identify and organize nodes into clusters, such that each cluster is a set of correlated data points. The proposed design exploits the high degree of correlation in SHM data. More emphasis is put on transmitting uncorrelated data from different clusters 


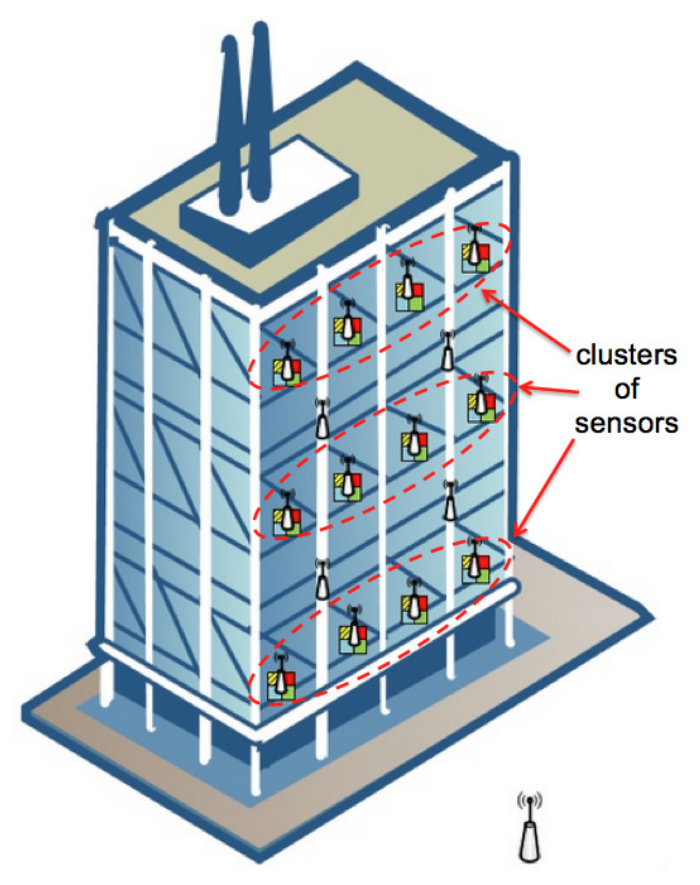

Figure 4.1: Deployment Scenario (figure adapted from picture on wireless building automation in wlba.wordpress.com)

evenly (Figure 4.2b) rather than allowing all sensor nodes to compete for channel access individually (Figure 4.2a).

The design sees each cluster as a supernode. As soon as one node of a cluster successfully transmits its data, the cluster is deemed to have succeeded in the current cycle. The rest of the nodes in that cluster refrain from further transmission until all the other clusters in the network have also succeeded in the current cycle. By the end of the cycle, one node from each cluster would have successfully transmitted its data packet.

When the PAN coordinator successfully receives a data packet from a node, it simply broadcasts an acknowledgement (ACK) packet containing the identifier of the successful node. The neighbour nodes in the cluster upon receiving this ACK get to know that a node in their cluster has succeeded. The nodes in this successful cluster transition into "active listening" mode, and the cluster as a whole is said to be in active listening 


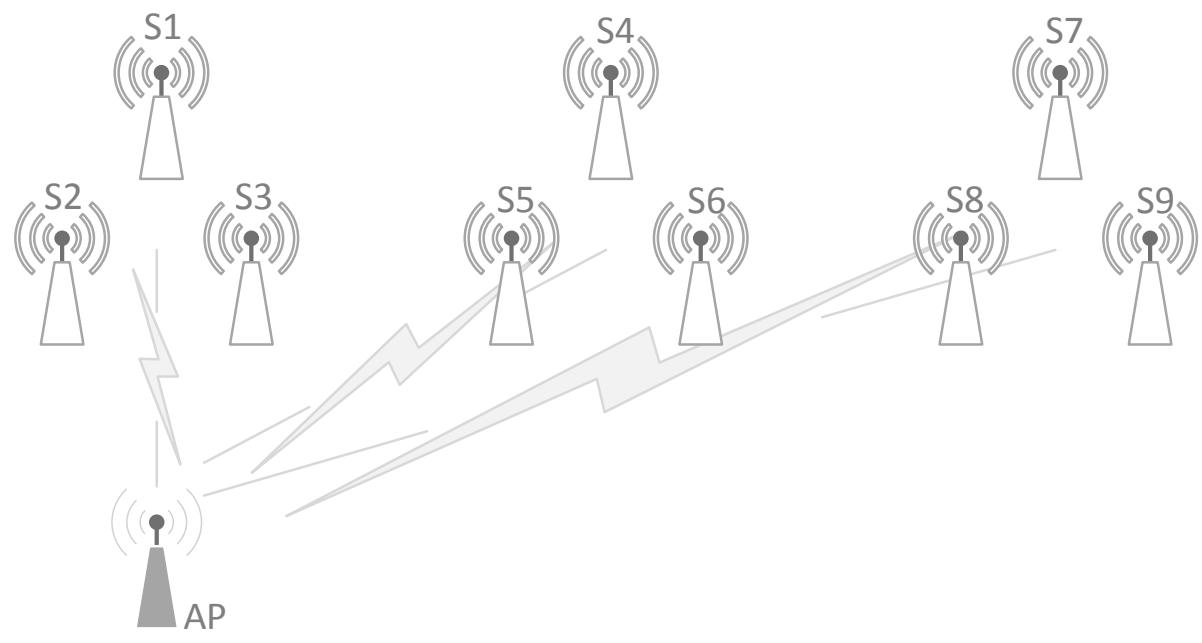

(a) Traditional Node Organization

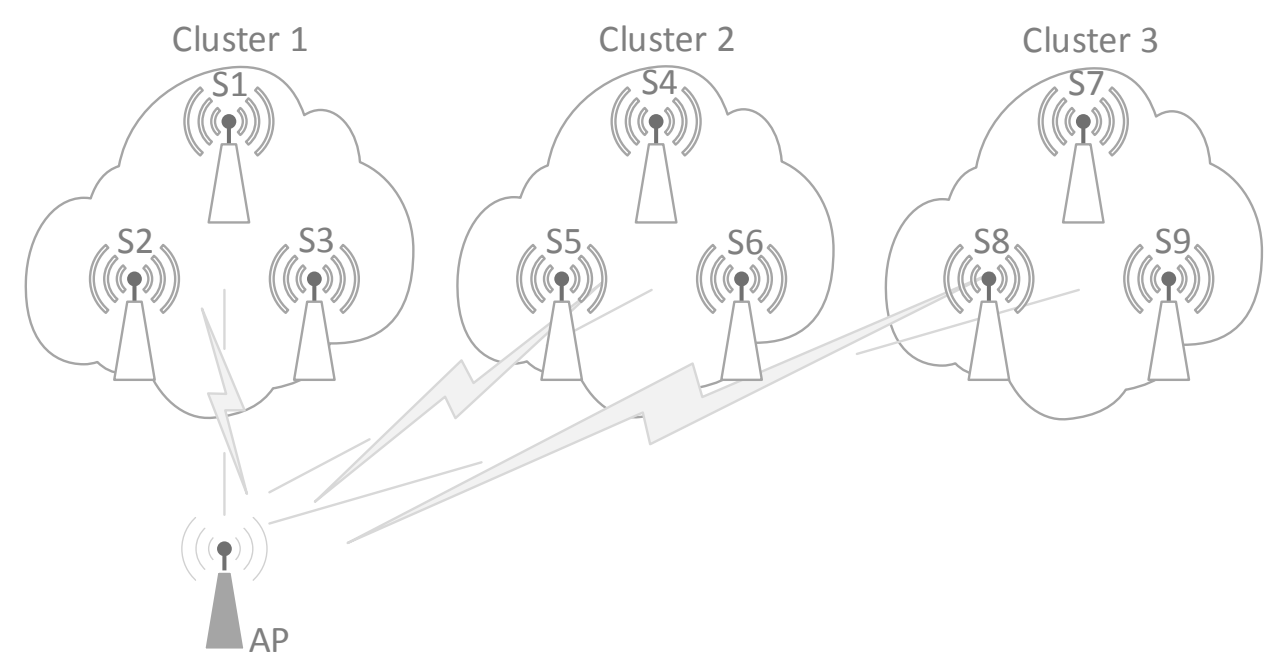

(b) Proposed Node Organization

Figure 4.2: Node Organization 
state. While active listening a node refrains from transmitting, but actively listens to broadcast signals from the PAN coordinator. Figure 4.3a shows the state transition diagram for the PAN coordinator.

The PAN coordinator typically remains idle until an event occurs(like earthquake) after which it waits for data from the sensor nodes. Although the PAN coordinator can remain in listening mode all the time as it is assumed to have sustained power source. It should be noted though that even minor tremors that cause low degree of structural vibrations can trigger an event and activate the sensors.

The algorithm is built on the standard IEEE802.15.4 non-beacon mode protocol, wherein the PAN coordinator is the sink node and solely relies on the unslotted CSMA/CA mechanism to arbitrate transmission attempts by the nodes.

A critical component of the IEEE802.15.4 MAC protocol is the Backoff Exponent (BE). Before a node attempts to transmit a packet, it first delays for a random number of backoff units in the range 0 to $2^{B E}-1$ and then checks that the channel is clear/idle before it transmits. This random number is selected based on a uniform distribution, which means every number in the range 0 to $2^{B E}-1$ has an equal chance of being selected by a node. In a network with many nodes wanting to transmit, this leads to a high probability of collision.

Motivated by the optimal backoff time slot selection algorithm proposed by Cheng et al. [62], the nodes are made to randomly select a number based on geometric distribution. Lower probability is assigned to smaller numbers so that fewer nodes transmit early. This reduces the chances of collisions, giving nodes a better chance at successful transmissions. There are existing researches $[73,74]$ proving that non-uniform channel selection reduces the number of network collisions compared to uniform channel selection. 


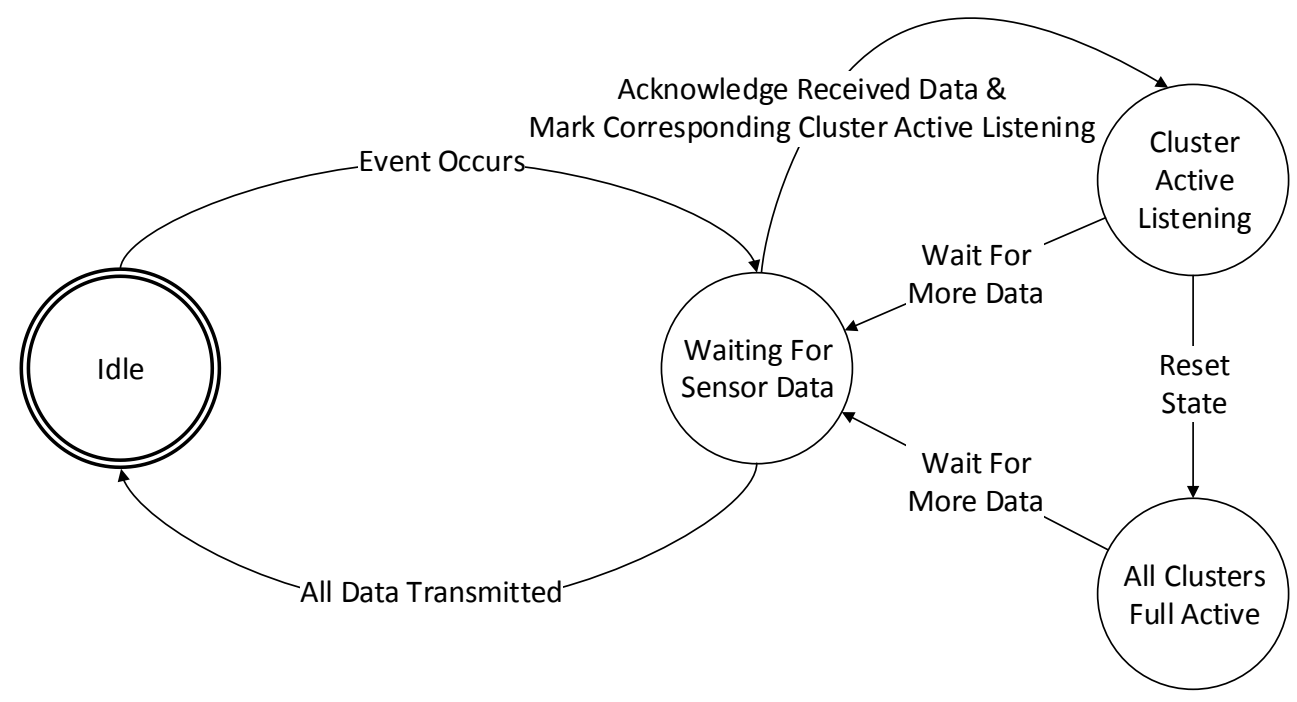

(a) PAN Coordinator

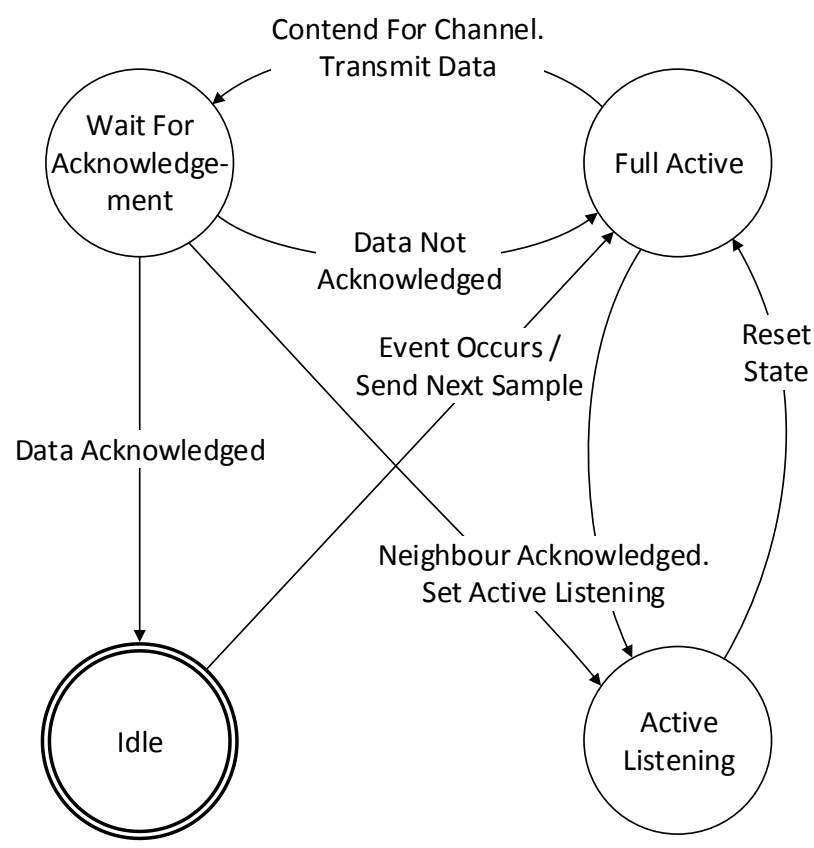

(b) Sensor Node

Figure 4.3: State Transition Diagrams 
Every time a (node in a) cluster successfully transmits a data packet, the cluster goes to active listening state thereby bringing down the network size by one cluster. This reduces the number of nodes contending for channel access subsequently, and increases the successful transmission probability of the remaining clusters. Depending on the cluster and network size, network contention drops rapidly with each successfully transmitted packet and considerably increases the successful transmission probability of remaining clusters.

When all the clusters have transmitted one data packet each and all the clusters are in active listening state, it is regarded as the end of a transmission cycle. The PAN coordinator then broadcasts a "reset" frame and the nodes that did not manage to successfully transmit their packets in the cycle that just ended try again in the next transmission cycle. This is the "Reset Cluster State" event that puts all the clusters back into "Full Active" state which is the lower-right bubble of the PAN coordinator state machine in Figure 4.3a and upper-right bubble in the Sensor Node state machine in Figure $4.3 \mathrm{~b}$. In the next transmission cycle, the network operates in the same manner as the previous transmission cycle with the only exception that the nodes which have already successfully transmitted their packets (in previous cycles) do not participate.

The decision to put a cluster to active listening state is taken autonomously at node level based on data acknowledgement broadcast by the PAN coordinator. Putting a node to active listening state simply means that the node refrains from contending for channel access to transmit, while the hardware state remains exactly as an active node. A node goes to active listening state when a neighbour node belonging to the same cluster is acknowledged for a successful packet transmission, and resets its state back to full active only when the PAN coordinator sends a reset signal as shown in Figure4.3b.

Once all the clusters are in active listening state, the PAN coordinator broadcasts a "reset" frame to reset all the nodes' state for next round of 
transmissions. To reduce the nodes' energy consumption, the PAN coordinator can also include in the ACK packet the number of remaining clusters to transmit and "active listening" nodes can estimate the quickest time required for these remaining clusters to successfully transmit, and then go to sleep for this period of time.

\subsection{Summary}

This chapter presented a cluster-centric MAC algorithm that allocates channel access to nodes within clusters based on an implicit-round robin. The proposed design has two key characteristics:

1. Higher priority is given to transmission of uncorrelated data from different clusters.

2. Nodes within a cluster have fair chance to transmit during each transmission cycle.

The design proposes to improve the performance of the standard IEEE 802.15.4 MAC protocol for bursty networks on the following grounds:

1. The implicit round-robin in the proposed design promises fairness among clusters of nodes. This results in elimination of network bias.

2. With every successful transmission a cluster is taken out of network contention. That coupled with geometric random backoff, should result in reduced contention, retransmission attempts, delay, and energy consumption. Which should further lead to faster successful transmissions and improved goodput. It can be assumed that such performance improvements should produce a scalable network.

The result evaluation in the next section will analyse the performance on these grounds both individually and combined, against the standard IEEE802.15.4 MAC protocol. 


\section{Chapter 5}

\section{Evaluation}

This chapter presents the performance evaluation of the proposed clustercentric MAC protocol using the QualNet 5.2 simulator. The comparison and validation of the proposed scheme is done against other IEEE802.15.4 variants as well as the WSN approach proposed by Liu et al. [33] that employs in-network processing of SHM data.

\subsection{Evaluation Model}

The study uses the standard IEEE802.15.4 protocol, and the proposed cluster-centric MAC is built on top of that. Varying cluster sizes $(5,10,15$, 20 , and 25) and network sizes (100, 150, 200, and 250) are used for the evaluation, resulting in twenty different combinations. To obtain accurate averages the result for each combination is an average of ten different runs, with each run using a different seed value.

The sensor nodes are placed as they would be in a real life SHM system, for example Figure 5.1. The PAN coordinator is usually outside the building and not very high above the ground, and the sensor nodes in the building with the ground floor sensor nodes closest to the PAN coordinator and the highest floor sensor nodes farthest away. Nodes in cluster \#1 are closest to the PAN coordinator and the cluster numbers increase with 


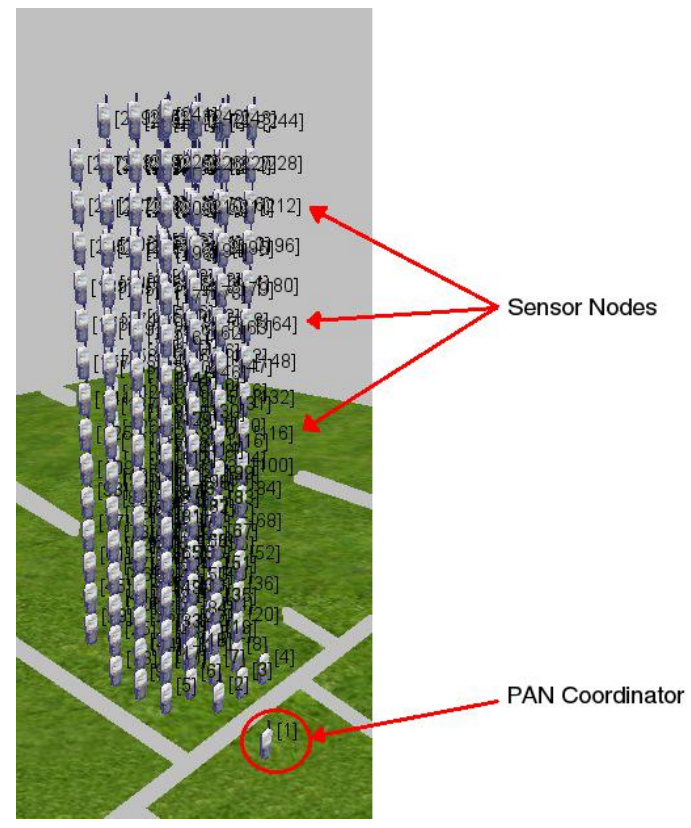

Figure 5.1: Example Qualnet Deployment Snapshot $(\mathrm{N}=250)$

increasing distance from the PAN coordinator. This deployment scenario is similar to that shown in Figure 4.1.

For the purpose of evaluation, the following assumptions are made:

- A fixed amount of event data is generated simultaneously (at time $t_{0}$ ) at all nodes, post a strong tremor or earthquake.

- As shown in Figure 5.1 the evaluation model assumes uniform distribution of nodes. The inter-node distance is set as 5 metres, and the distance between the building and the PAN coordinator is set as 15 metres.

- The model uses a simple battery with $2400 \mathrm{mAh}$ initial charge, and the MICAZ Qualnet energy model.

- The PAN coordinator is set as FFD and all the other devices as RFDs. 
- The physical and MAC layer use the default Qualnet 802.15.4 radio and reception model.

\subsection{Result Evaluation}

Traditional comparison metrics have evaluated WSN performance from node/network perspective like transmission time, delay, energy, retransmissions, throughput, goodput, etc. This thesis evaluates the results from a new cluster-centric perspective, wherein performance is evaluated based on packet arrival time from the clusters.

\subsubsection{Cluster-Centric Result Evaluation}

The simulation parameters used are shown in Table 5.1.

\begin{tabular}{|c|c|}
\hline Parameter & Value \\
\hline Network Size & $100,150,200,250$ \\
\hline Cluster Size & $5,10,15,20,25$ \\
\hline Data Packets Per Node & 1 \\
\hline Total Network Data Packets & $100,150,200,250$ \\
\hline MAC Algorithm & $\begin{array}{c}\text { Standard IEEE802.15.4, } \\
\text { Cluster-Centric MAC }\end{array}$ \\
\hline Random Number Generator & Uniform, Geometric \\
\hline
\end{tabular}

Table 5.1: Simulation Parameters For Cluster-Centric Comparison

\subsubsection{Comparing Fairness}

The results show only one set of representative results for each network size from a series of twenty different combinations, since all combinations produce similar set of results. Results with 10 cluster count are shown, to 


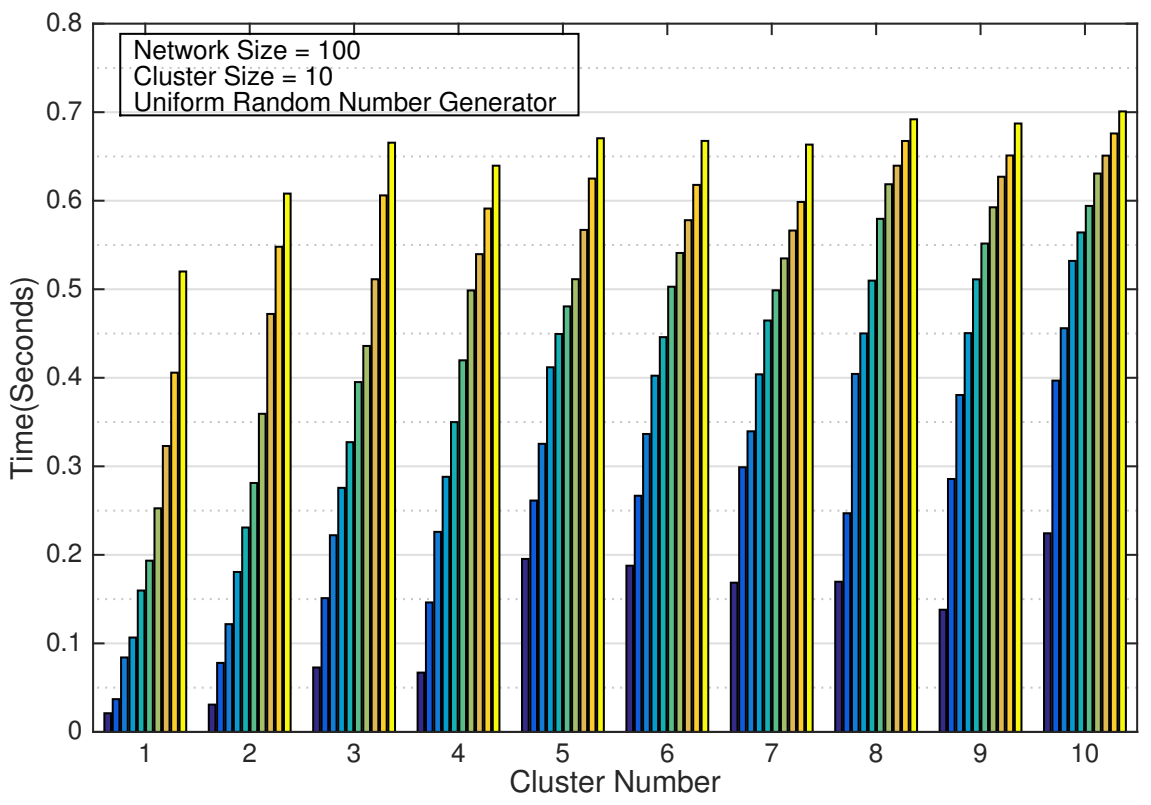

(a) IEEE802.15.4 MAC Protocol

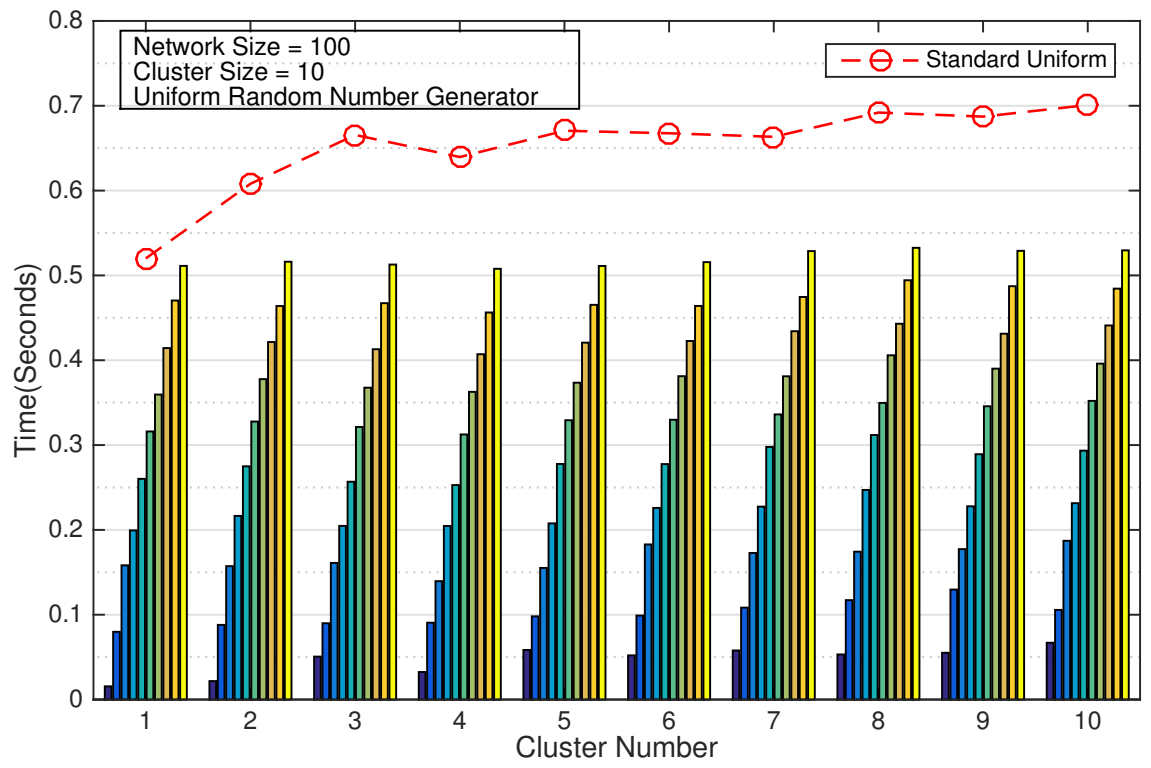

(b) Cluster-Centric MAC Protocol

Figure 5.2: Comparing Fairness $(\mathrm{N}=100, \mathrm{C}=10)$ 


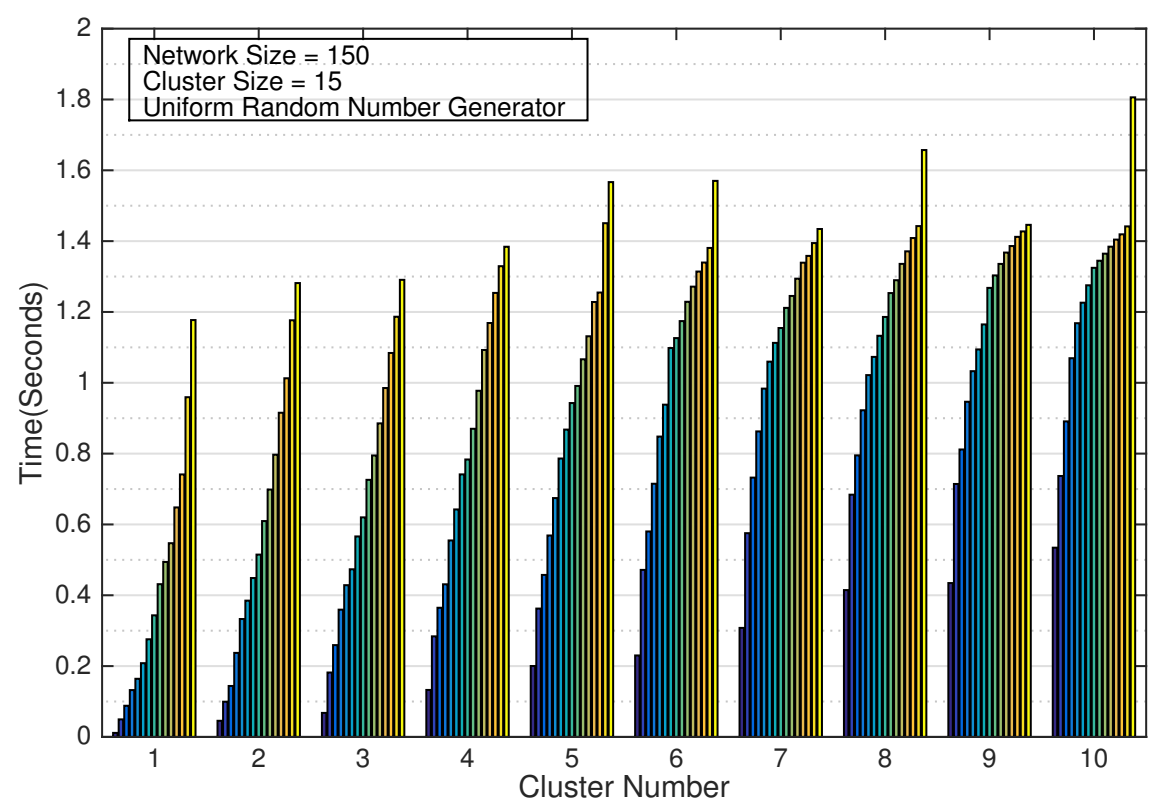

(a) IEEE802.15.4 MAC Protocol

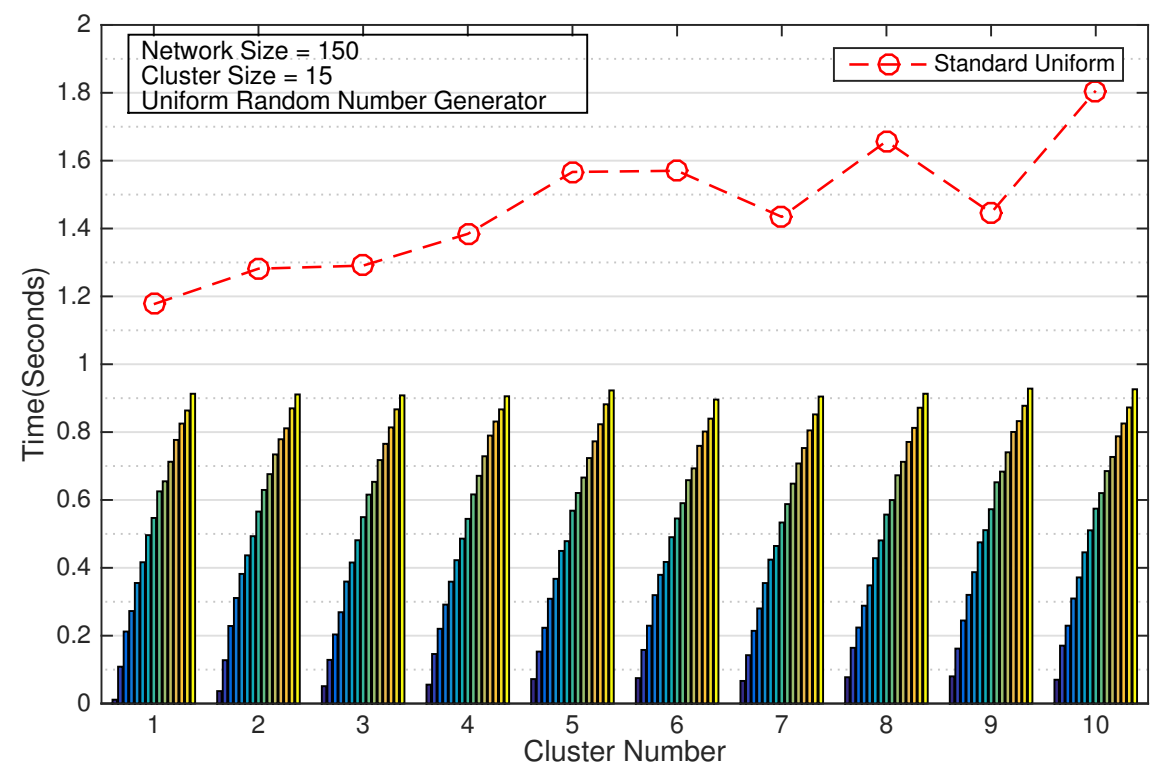

(b) Cluster-Centric MAC Protocol

Figure 5.3: Comparing Fairness $(\mathrm{N}=150, \mathrm{C}=15)$ 


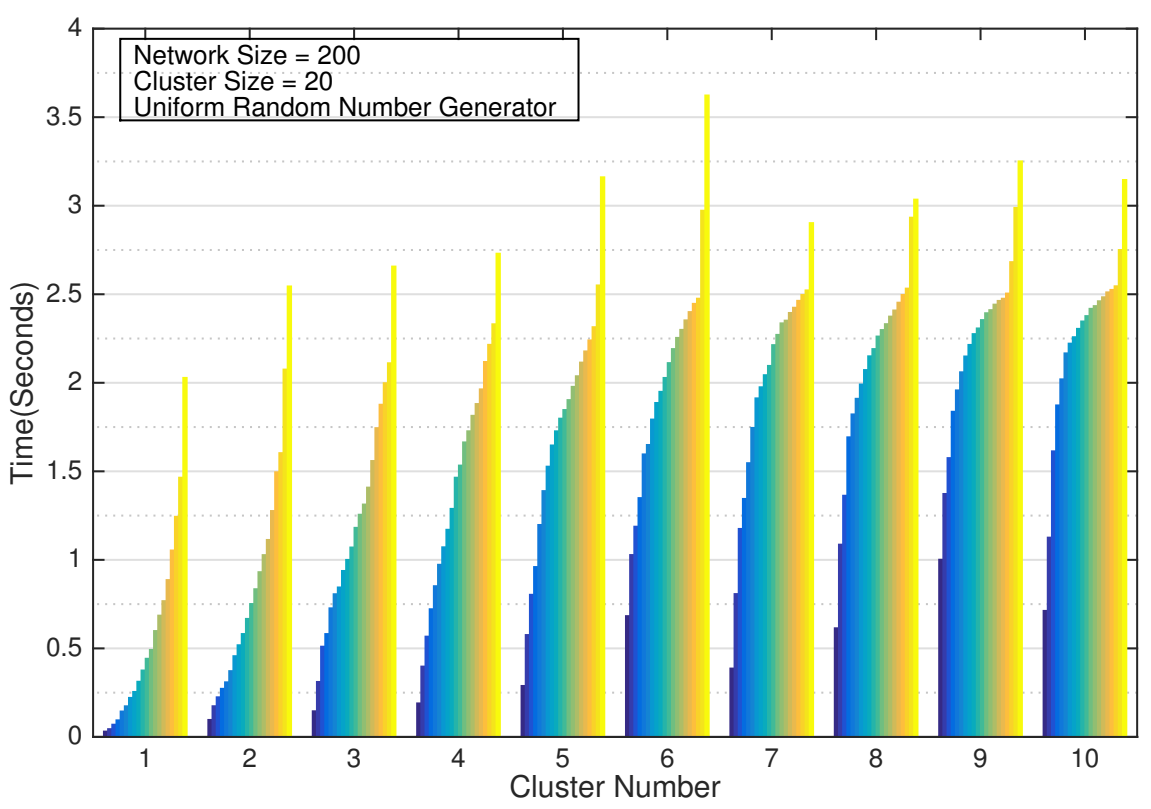

(a) IEEE802.15.4 MAC Protocol

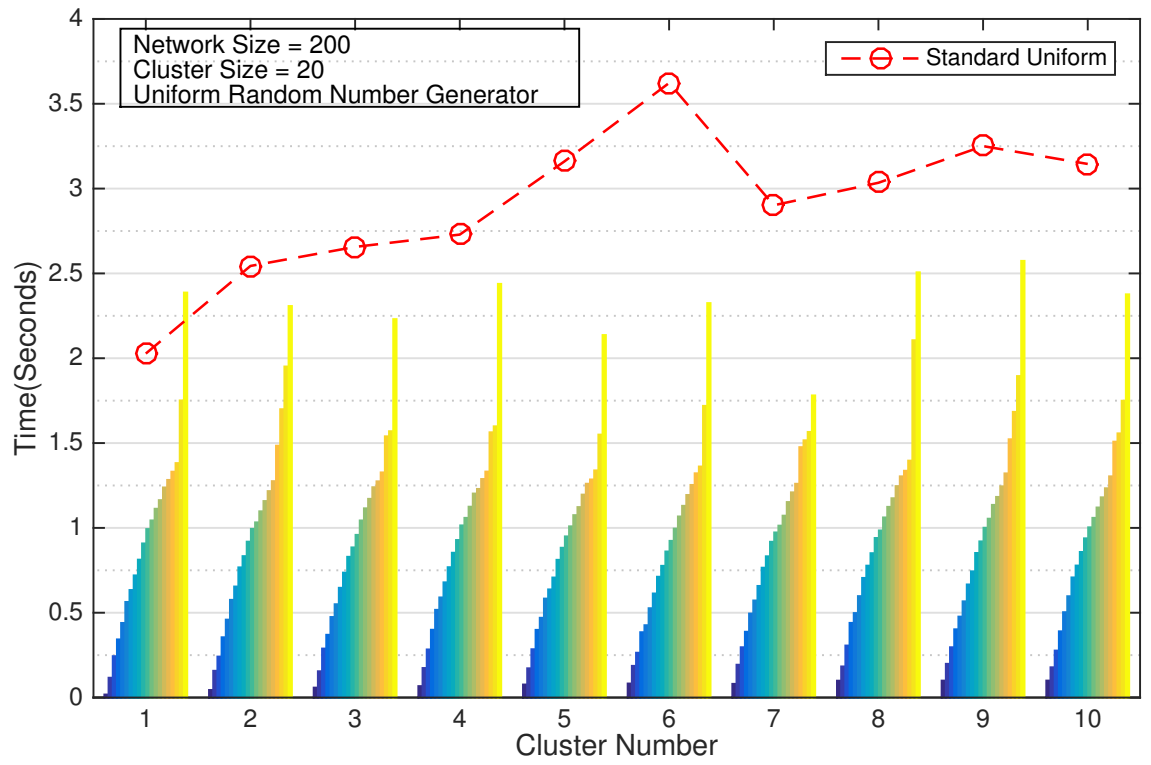

(b) Cluster-Centric MAC Protocol

Figure 5.4: Comparing Fairness $(\mathrm{N}=200, \mathrm{C}=20)$ 


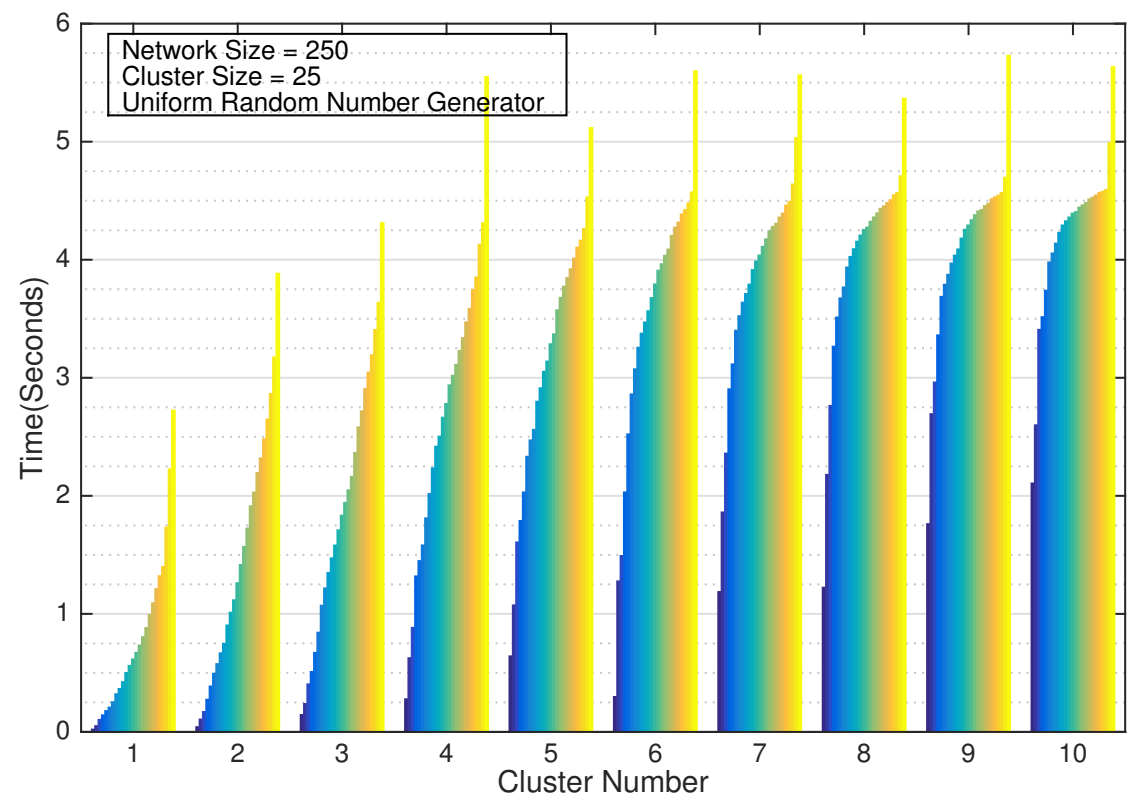

(a) IEEE802.15.4 MAC Protocol

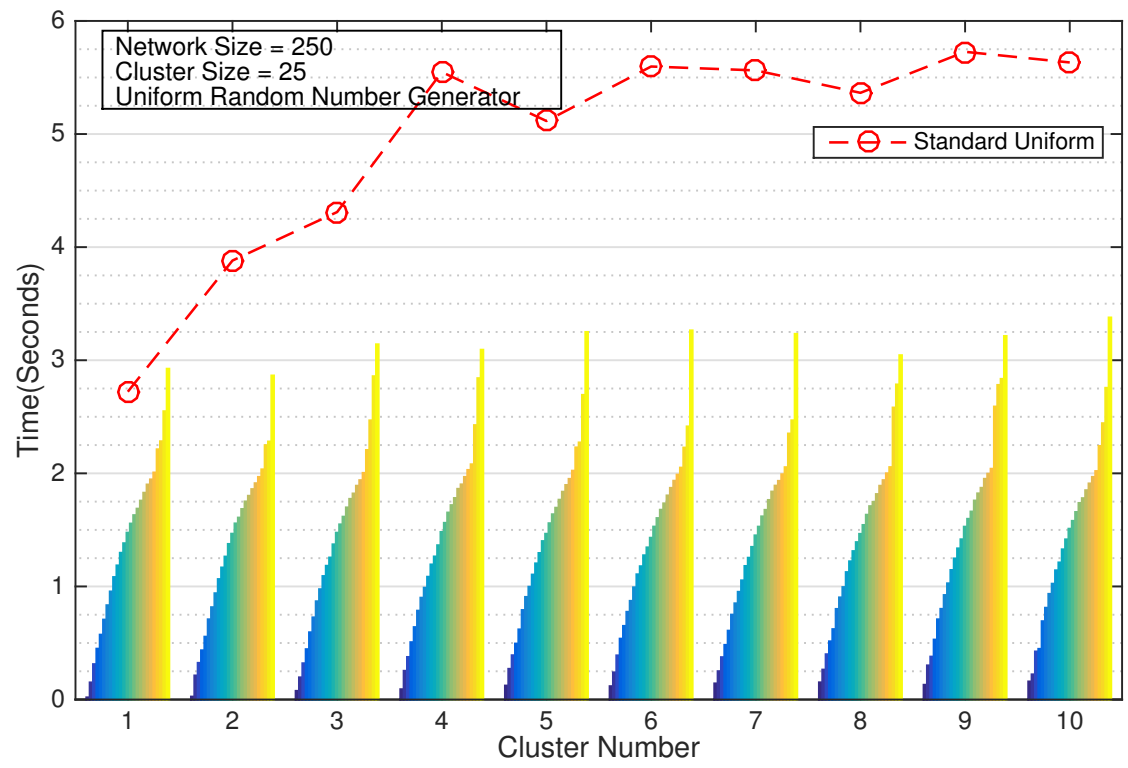

(b) Cluster-Centric MAC Protocol

Figure 5.5: Comparing Fairness $(\mathrm{N}=250, \mathrm{C}=25)$ 
show the packet delivery characteristics of the IEEE802.15.4 MAC and the cluster-centric MAC protocols.

Referring to Figures 5.2, 5.3, 5.4, and 5.5, the vertical plots for each cluster show the time duration (since $t_{0}$ ) at which consecutive packets within a cluster are successfully transmitted and received by the PAN coordinator; e.g., the blue bar on the left shows the time needed by the first successfully transmitted packet of a cluster (irrespective of which sensor within the cluster it came from) and the next bar shows the second successful packet, and so forth. The red dotted line represents the time taken by the standard IEEE802.15.4 protocol (with uniform backoff) to complete all cluster data transmission.

It should be noted though that the IEEE802.15.4 MAC protocol does not have the concept of clusters (as shown in Figures 5.2a, 5.3a, 5.4a, and 5.5a), and the output irrespective of cluster size will always be the same. The thesis, just for comparative evaluation organizes the nodes into clusters and observes the arrival time using the standard IEEE802.15.4 MAC protocol.

In Figures 5.2a, 5.3a, 5.4a, and 5.5a, the standard IEEE802.15.4 MAC protocol produces bias towards nodes and clusters closer to the PAN coordinator. This skewed performance for nodes/clusters closer to the PAN coordinator can be attributed to capture effect [28] which has been observed and studied in IEEE802.15.4 networks $[75,76]$. This leads to clusters that are closer to the PAN coordinator node being able to transmit all their data much sooner than the clusters that are farther away.

The proposed model eliminates this bias phenomenon by ensuring that each cluster gets a fair chance rather than individual nodes. This is achieved by taking a cluster (and all the nodes therein) out of the contention for the channel once it is successful in the current cycle. No bias results are observed, as reflected in Figures 5.2b, 5.3b, 5.4b, and 5.5b which shows every cluster evenly sending packets to the PAN coordinator. Since there is no bias among clusters, all the clusters finish transmitting their data 
around the same time. This is a favourable consequence of the clustercentric approach which reduces overall network contention and improves the entire network's performance.

\subsubsection{Comparing Speed}

Both Figures 5.2a, 5.3a, 5.4a, and 5.5a and Figures 5.6a, 5.7a, 5.8a, and 5.9a use the same IEEE802.15.4 CSMA/CA MAC algorithm but different random number generator. However, the geometric random backoff finishes faster than uniform random backoff. The same can be observed in Figures $5.2 b, 5.3 b, 5.4 b$, and $5.5 b$ and Figures $5.6 b, 5.7 b, 5.8 b$, and $5.9 b$ for the cluster-centric MAC.

The red, blue, and black dotted lines represent the time taken to complete all the cluster data transmission by the standard IEEE802.15.4 protocol with uniform random backoff, proposed cluster-centric protocol with uniform random backoff, and the standard IEEE802.15.4 protocol with geometric random backoff respectively.

It is reasoned that by choosing a larger initial backoff there will be fewer collisions. Fewer collisions means nodes need not exponentially backoff, thus reducing the average time to complete transmitting the information generated by the event.

In the proposed model, once a node is able to successfully transmit its data packet, the corresponding cluster refrains from further transmission until the next transmission round, which helps in significant drop in contention as fewer nodes have to contend for channel access after a successful data transfer. This, coupled with geometric backoff time slot selection, further reduces contention which results in less overall contention and faster transmission times.

The proposed design permits only one data packet transmission per node per transmission round. This subsection aimed to show network behaviour and performance at micro level in one such transmission round. 


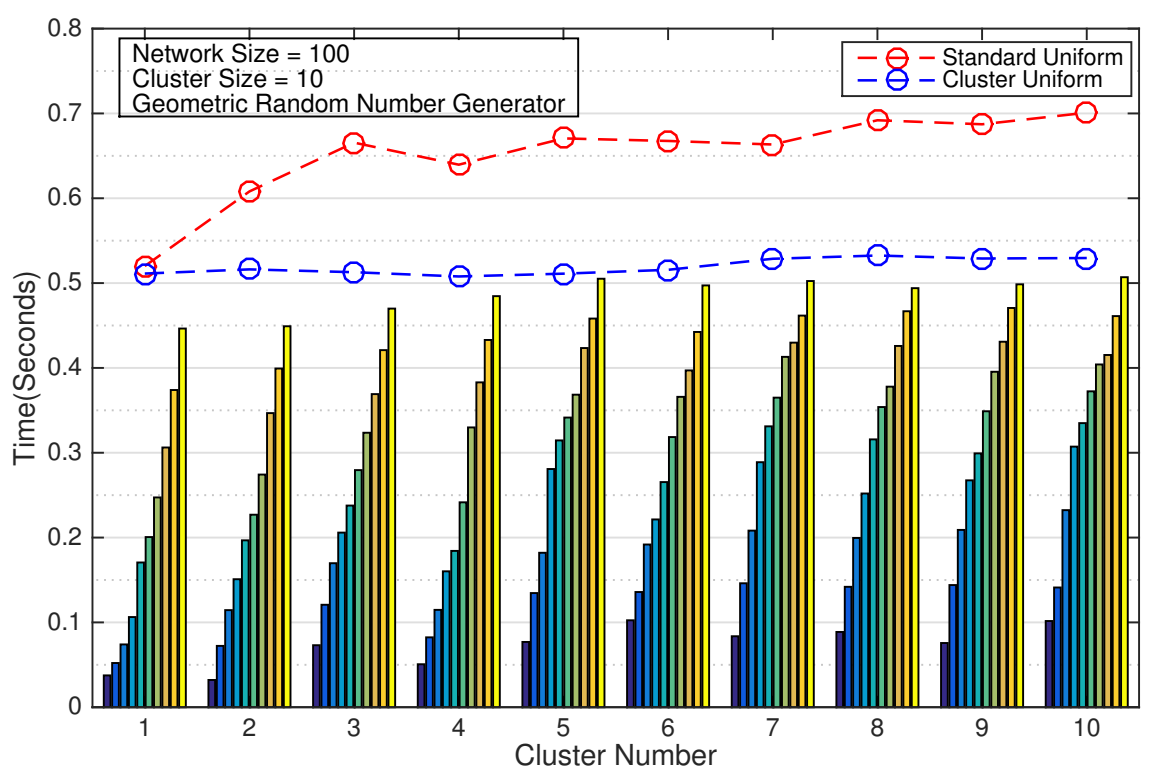

(a) IEEE802.15.4 MAC Protocol

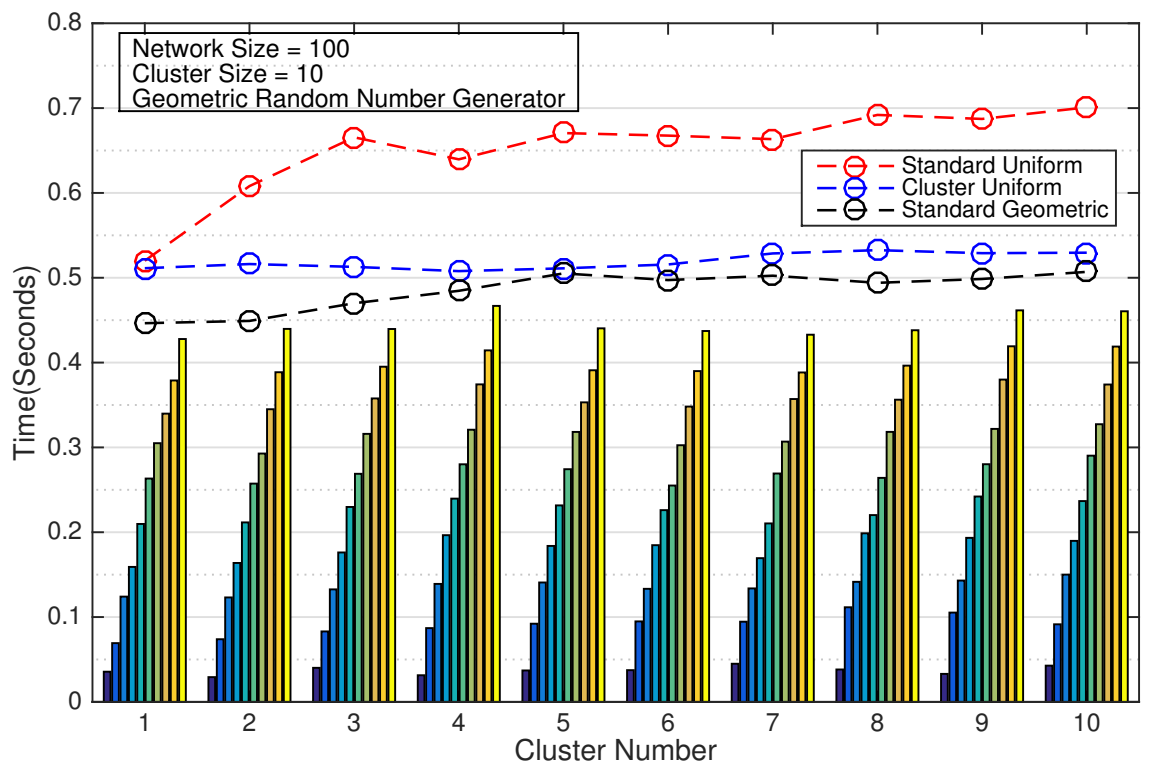

(b) Cluster-Centric MAC Protocol

Figure 5.6: Comparing Speed $(\mathrm{N}=100, \mathrm{C}=10)$ 


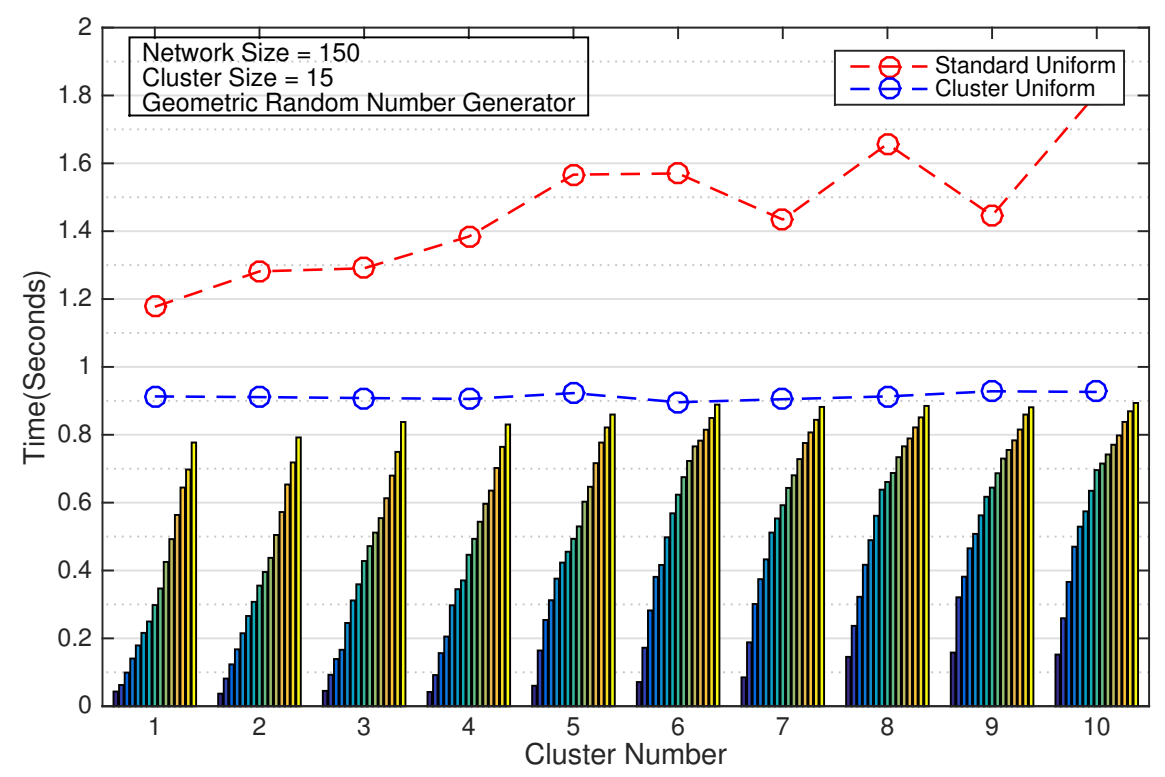

(a) IEEE802.15.4 MAC Protocol

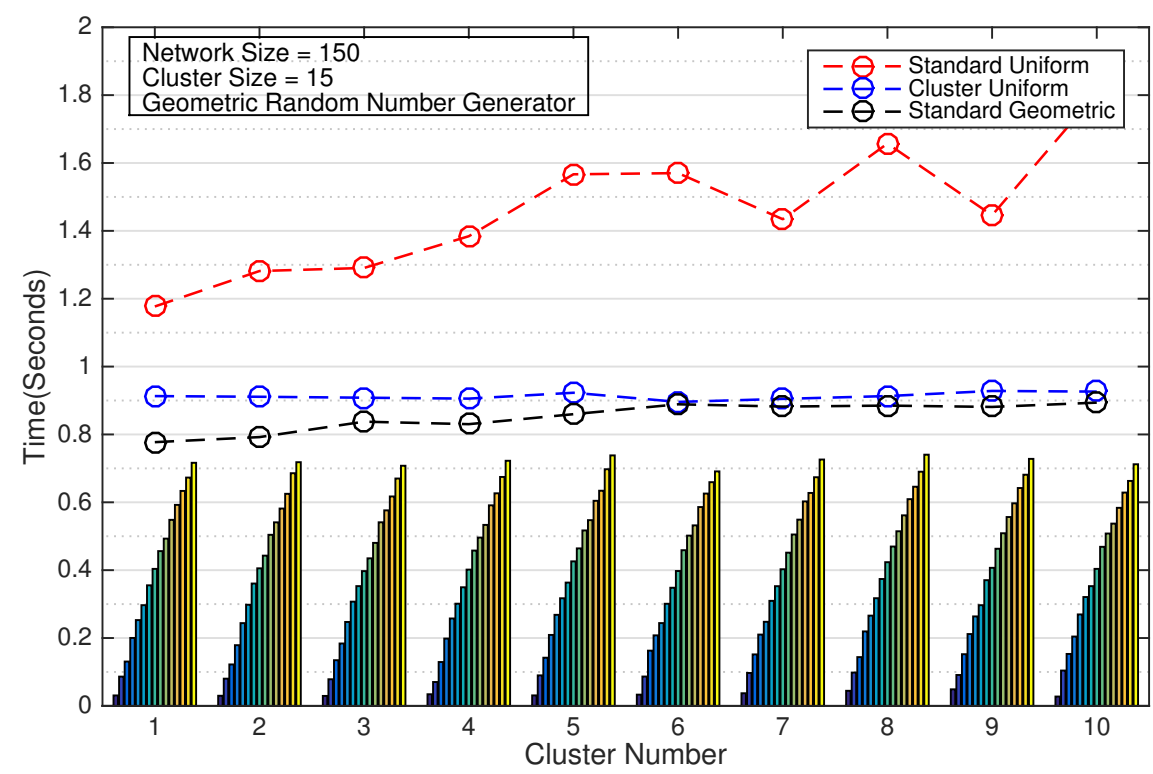

(b) Cluster-Centric MAC Protocol

Figure 5.7: Comparing Speed $(\mathrm{N}=150, \mathrm{C}=15)$ 


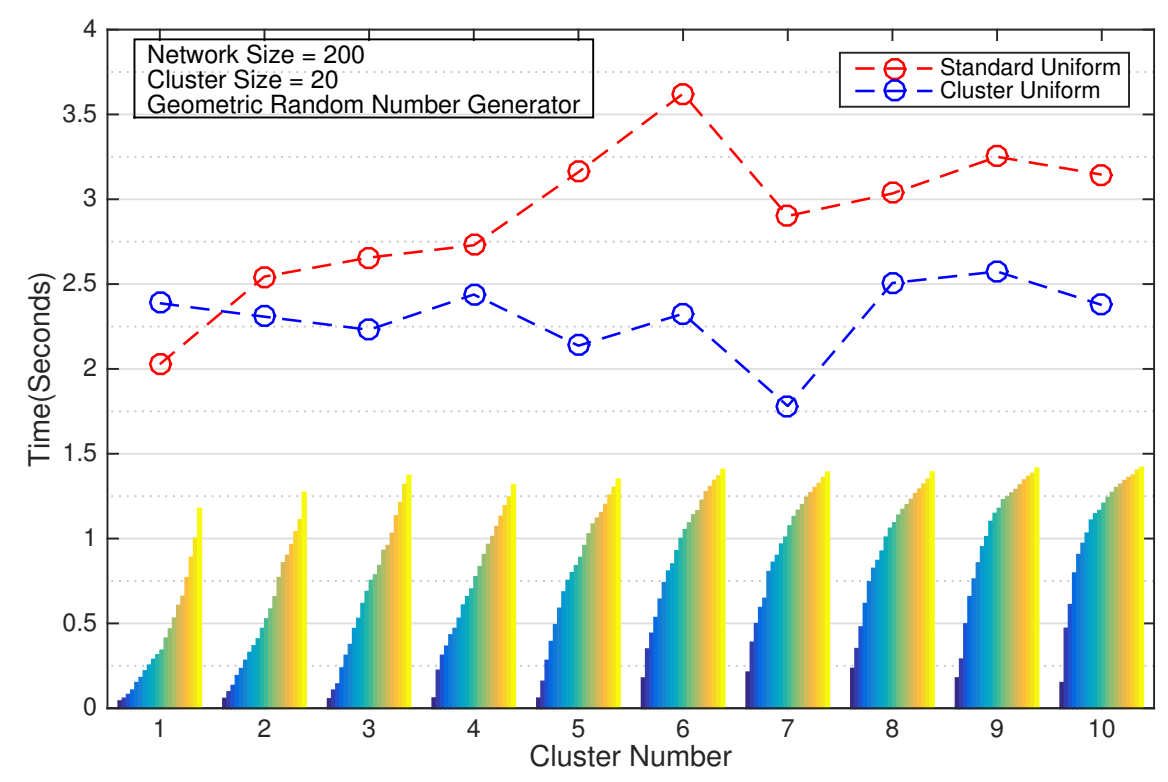

(a) IEEE802.15.4 MAC Protocol

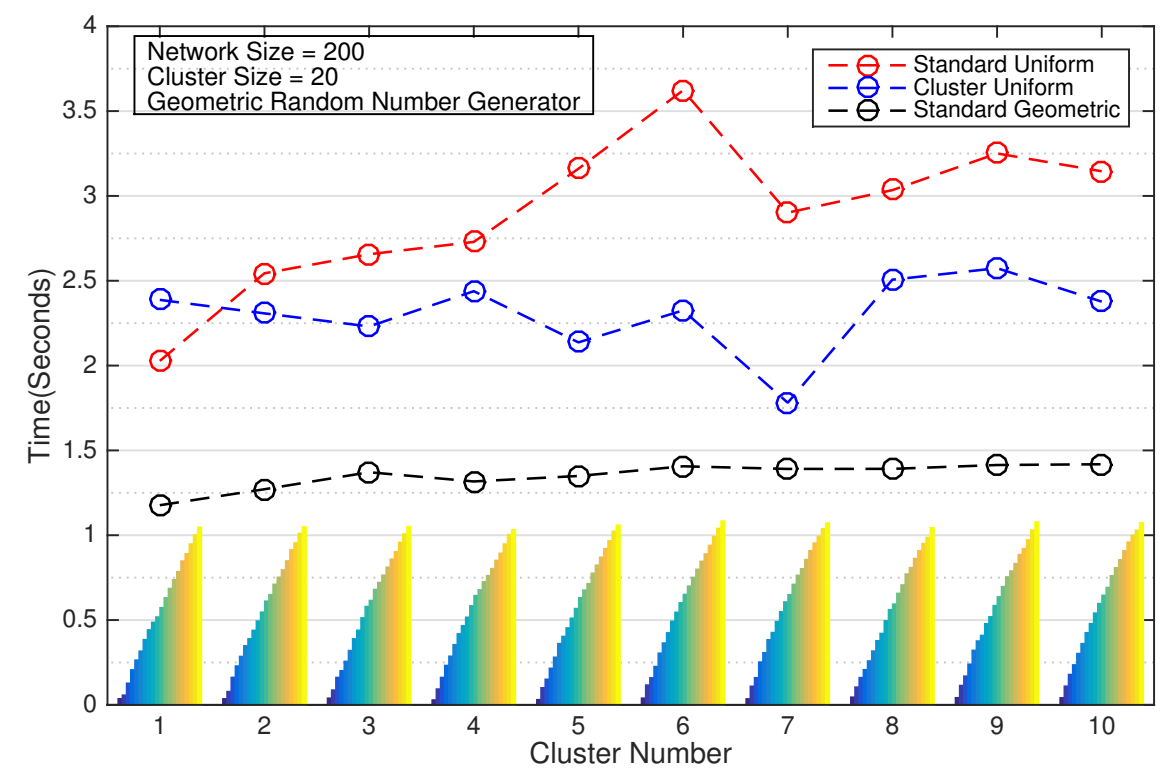

(b) Cluster-Centric MAC Protocol

Figure 5.8: Comparing Speed $(\mathrm{N}=200, \mathrm{C}=20)$ 


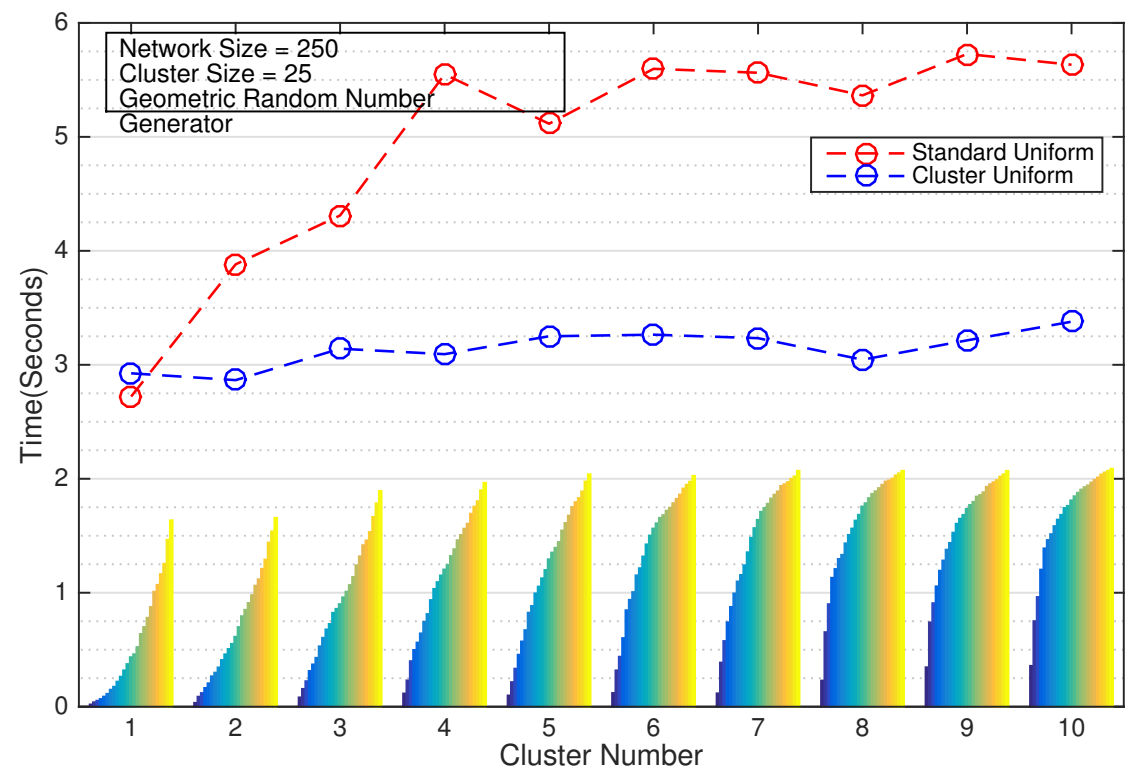

(a) IEEE802.15.4 MAC Protocol

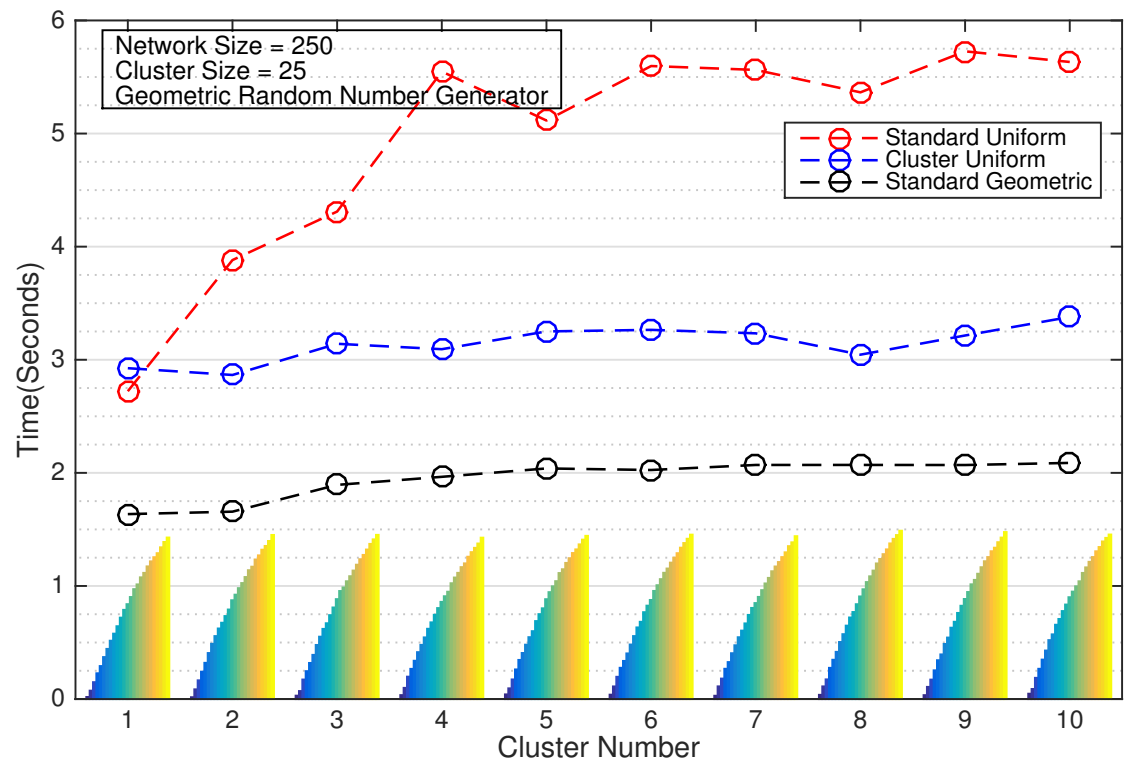

(b) Cluster-Centric MAC Protocol

Figure 5.9: Comparing Speed $(\mathrm{N}=250, \mathrm{C}=25)$ 
Real SHM systems typically have multiple samples per node $[26,33,77$, $78,79]$, hence it is natural for nodes to have multiple data packets to send. The next subsection shows network behaviour and performance at both micro and macro level.

\subsubsection{Node/Network Wide Result Evaluation}

While the aim of this thesis is to study the problem from cluster-centric perspective and focus on unbiased distribution of data, this subsection aims to show some related results from traditional network-wide perspective.

\subsubsection{Comparing The Averages}

This section compares the average time to transmit all packets and the average retransmission count for different cluster and network size combinations. Table 5.2 shows the simulation parameters used.

\begin{tabular}{|c|c|}
\hline Parameter & Value \\
\hline Network Size & $100,150,200,250$ \\
\hline Cluster Size & $5,10,15,20,25$ \\
\hline Data Packets Per Node & 1,215 \\
\hline Total Network Data Packets & $\begin{array}{c}100,150,200,250 \\
21500,32250,43000,53750\end{array}$ \\
\hline MAC Algorithm & $\begin{array}{c}\text { Standard IEEE802.15.4, } \\
\text { Cluster-Centric MAC }\end{array}$ \\
\hline Random Number Generator & Uniform, Geometric \\
\hline
\end{tabular}

Table 5.2: Simulation Parameters For Comparing The Averages 


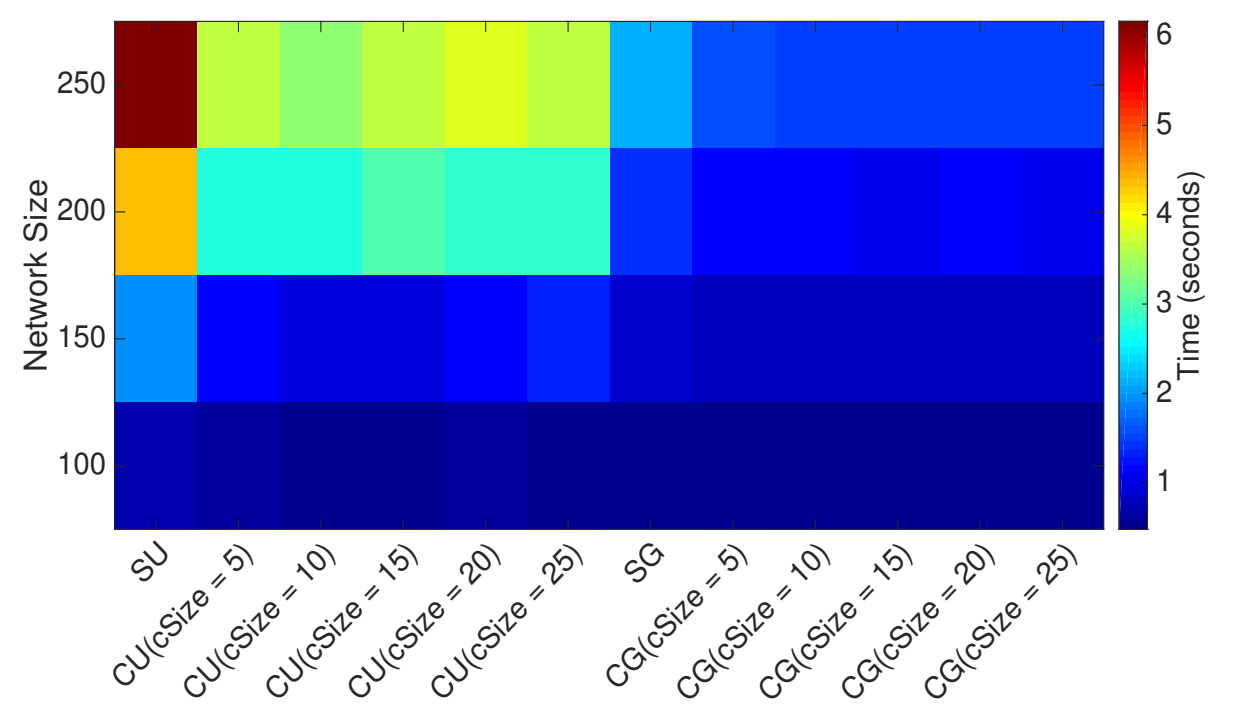

(a) Average Time To Transmit All Packets.

SU : Standard IEEE802.15.4 - Uniform Random Number Generator $S G$ : Standard IEEE802.15.4 - Geometric Random Number Generator $C U$ : Cluster-Centric MAC - Uniform Random Number Generator CG : Cluster-Centric MAC - Geometric Random Number Generator cSize : cluster size

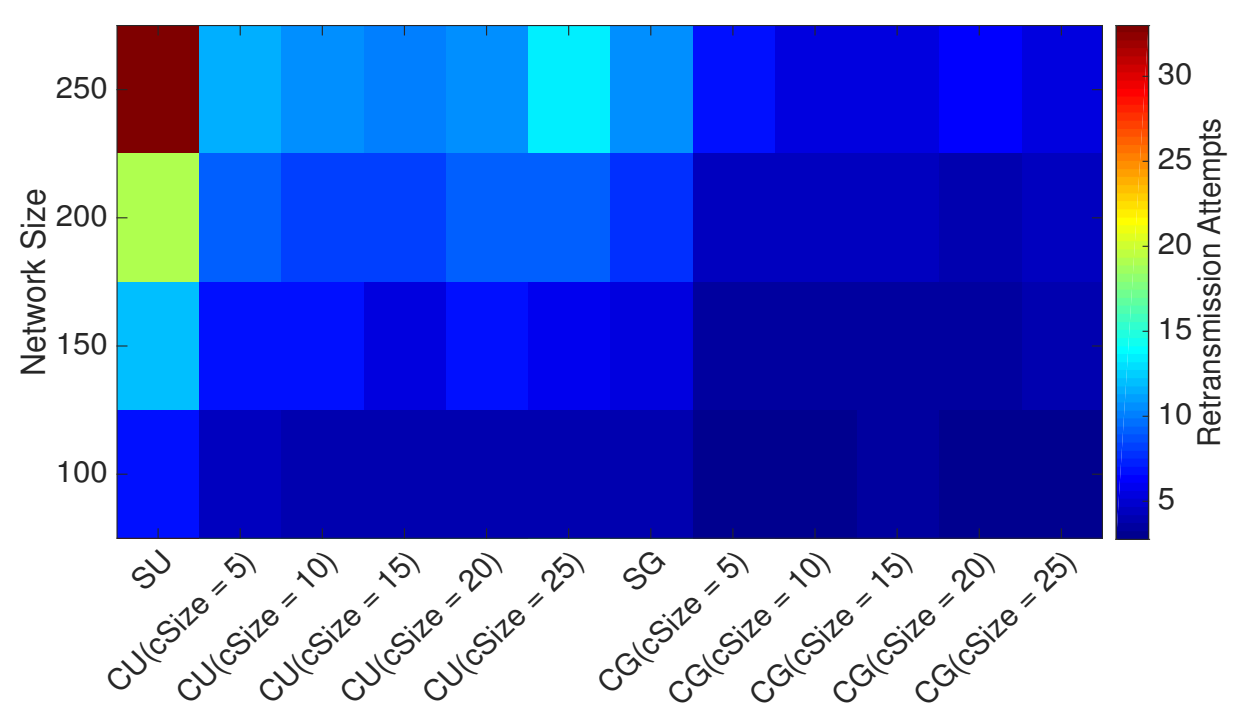

(b) Average Retransmission Attempts To Transmit All Packets.

Figure 5.10: Average Performance For Single Data Packet Per Node 
While it is evident from Figures 5.2 to 5.9 that the proposed scheme ensures unbiased transmission opportunities for clusters, the results also show that the event data is transmitted mush faster than the standard IEEE802.15.4 protocol. Consequently it should mean that the average time to transmit all the network event data should be on average shorter with the cluster-centric MAC, and the same is reflected in Figure 5.10a.

The results show that the time difference for small network sizes is very small, but it becomes more significant with increasing network size due to higher contention. While the standard IEEE802.15.4 protocol shows large variation in time to completion, the proposed model gives consistent time to complete transmissions irrespective of network size.

Retransmissions are costly actions in terms of time and energy, hence one of the aims of WSNs is to minimize retransmission count. The proposed design is able to significantly reduce the network congestion by taking nodes out of the contention from the channel promptly and hence is able to transmit all its data packets much sooner than the IEEE802.15.4 protocol. Less contention in return means less retransmissions, and the same effect of fewer retransmissions can be observed in Figure 5.10b.

It can be observed that the proposed design maintains consistent performance with increasing network size, and hence answers the scalability problem wireless networks usually deal with. A good network should be scalable not just in terms of network size but also in terms of network load. As previously stated, SHM systems usually have multiple data packets to send, hence network load scalability is a significant design issue.

The result variation for the IEEE802.15.4 protocol in Figure 5.10 increases many folds with increased network load as can be seen in Figure 5.11. The implicit round robin in the proposed design however ensures consistent performance irrespective of network size even at higher network load (Figure 5.11). This result shows the scalability of the proposed design with respect to network load. 


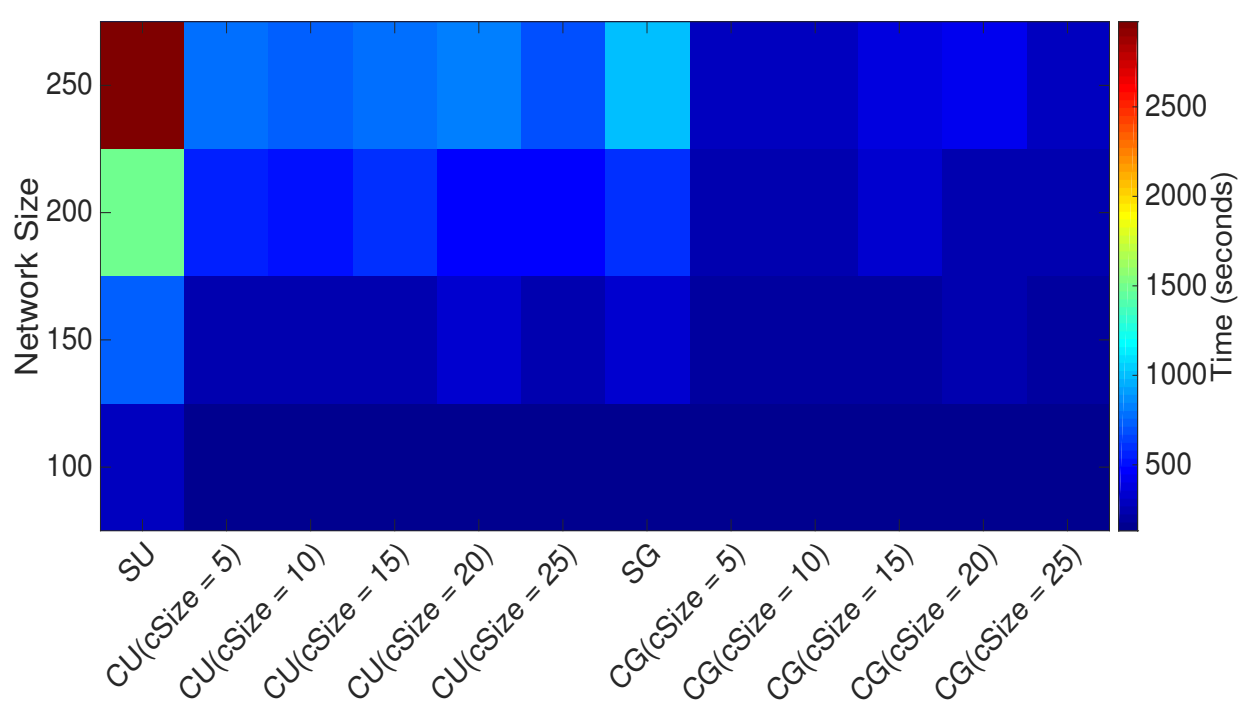

(a) Average Time To Transmit All Packets.

SU : Standard IEEE802.15.4 - Uniform Random Number Generator $S G$ : Standard IEEE802.15.4 - Geometric Random Number Generator $C U$ : Cluster-Centric MAC - Uniform Random Number Generator CG : Cluster-Centric MAC - Geometric Random Number Generator cSize : cluster size

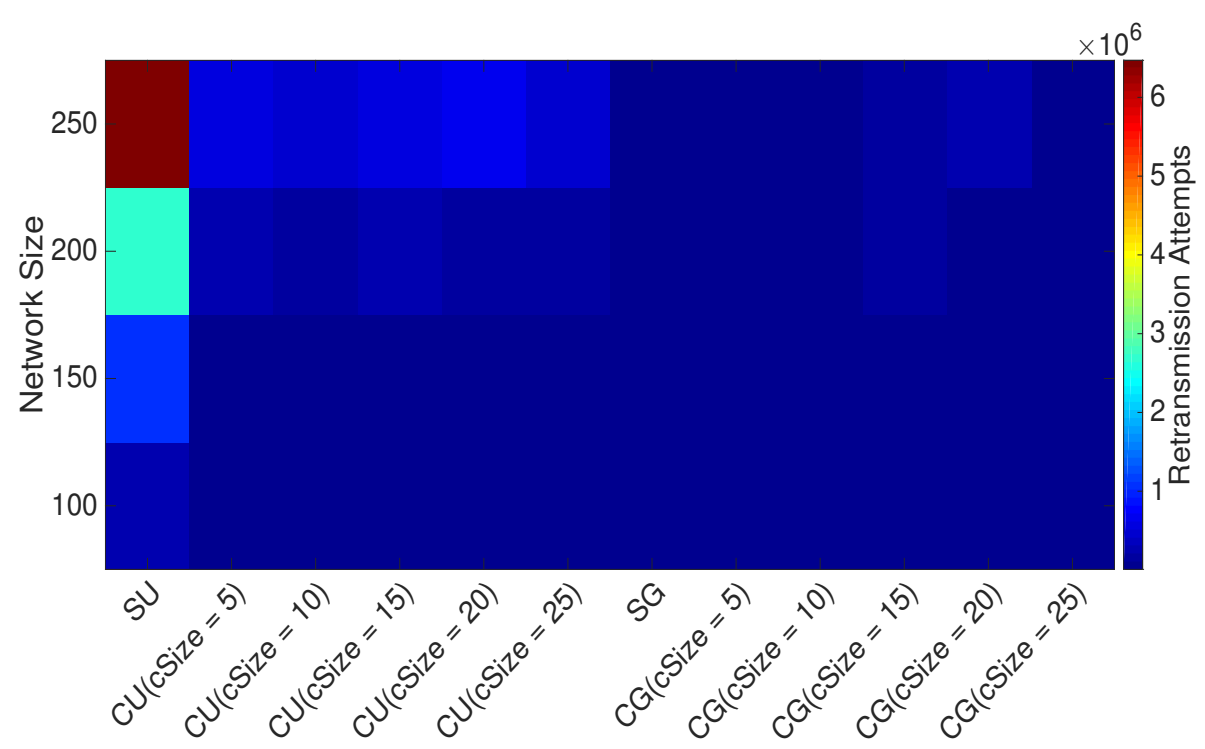

(b) Average Retransmission Attempts To Transmit All Packets.

Figure 5.11: Average Performance For Multiple Data Packets Per Node 


\subsubsection{Comparing The Throughput and Goodput}

Primary measure for effective resource allocation in wireless networks is through throughput comparison. This section compares the throughput and goodput. The throughput is measured in number of data packets transmitted per second, per sensor node. The goodput is measured in number of data packets successfully received per second at the sink node.

Ideally it is desirable to have higher throughput and goodput, but with bursty networks a higher throughput should lead to decreased goodput due to frequent collisions and packet loss. In a bursty network, it is important to control the throughput such that there are less collisions and packet loss in the network. The proposed scheme inhibits collisions by restraining neighbour nodes in a cluster to transmit after a successful transmission, thereby significantly reducing channel contention. Less collisions in effect should increase the goodput.

Table 5.3 shows the simulation parameters used, and Figure 5.12 shows the observed results. As expected the proposed scheme reduces the network throughput to reduce packet collisions, as can be observed in Figure 5.12a. The increased goodput observed at the PAN coordinator shows direct correlation with the throughput, as shown in Figure 5.12b.

\begin{tabular}{|c|c|}
\hline Parameter & Value \\
\hline Network Size & 250 \\
\hline Cluster Size & $5,10,15,20,25$ \\
\hline Data Packets Per Node & 215 \\
\hline Total Network Data Packets & 53750 \\
\hline MAC Algorithm & $\begin{array}{c}\text { Standard IEEE802.15.4, } \\
\text { Cluster-Centric MAC }\end{array}$ \\
\hline Random Number Generator & Uniform, Geometric \\
\hline
\end{tabular}

Table 5.3: Simulation Parameters For Throughput, Goodput and Delay Comparison 


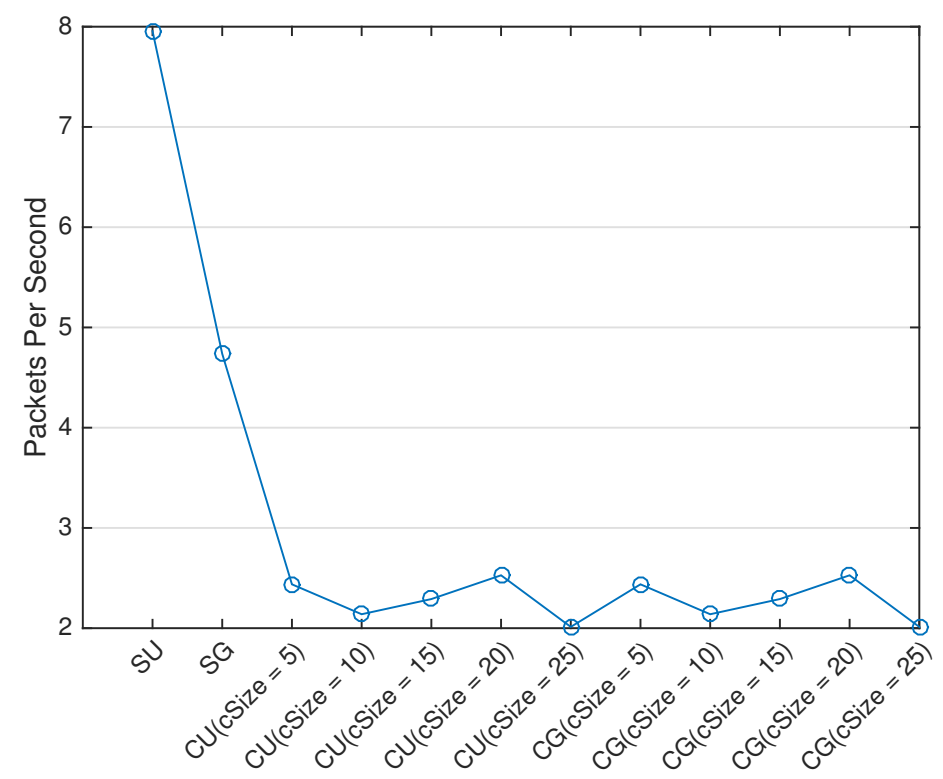

(a) Average Throughput Per Sensor Node

SU : Standard IEEE802.15.4 - Uniform Random Number Generator $S G$ : Standard IEEE802.15.4 - Geometric Random Number Generator $C U$ : Cluster-Centric MAC - Uniform Random Number Generator $C G$ : Cluster-Centric MAC - Geometric Random Number Generator cSize : cluster size

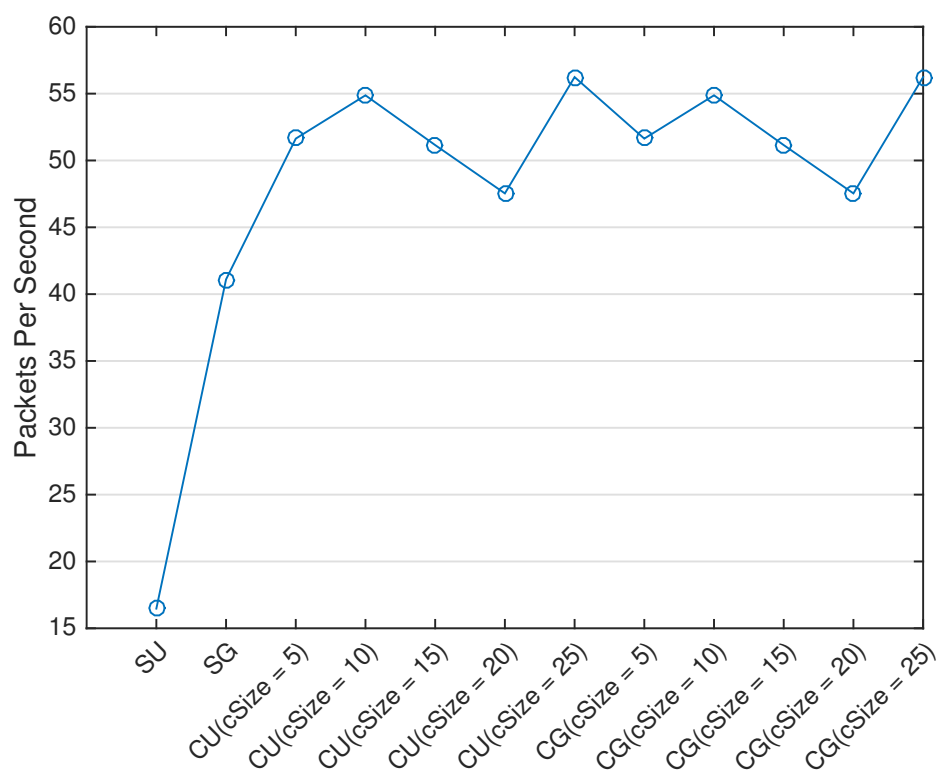

(b) Goodput At PAN Coordinator

Figure 5.12: Throughput vs Goodput (N=250) 


\subsubsection{Comparing Delay}

A bursty network leads to increased delay in successful packet delivery. Frequent collisions and packet loss in bursty network lead to delayed successful transmission. As observed in the previous results, the proposed design decreases the average throughput and retransmission attempts, and also improves the goodput leading to faster completion time. In theory this should mean that the proposed design should also decrease the average packet delay compared to the standard IEEE802.15.4 protocol.

As observed in Figures 5.13a and 5.14a the proposed scheme leads to significant decrease in delay per packet. The line plot shows the average delay per packet, and the boxplot shows the distribution characteristics of the packet delay. It is worth noting that the average packet delay and the median packet delay overlap for the proposed scheme.

Equal mean and median values often mean that the data set is equally spaced, but it is not always the case. For example data set $\{3,5,7,9,11\}$ and $\{3,4,7,10,11\}$, both have mean and median 7 , but only one of it is equally spaced.

The density distribution plot in Figure $5.13 \mathrm{~b}$ and $5.14 \mathrm{~b}$ shows that the proposed scheme follows a uniform distribution which confirms that the delay is indeed equally spaced. This means that the arrival time at the PAN coordinator is also equally spaced. This signifies sustained use of channel. The density distribution of the IEEE802.15.4 MAC protocol on the contrary shows that the packet delay is not equally spaced out and most of the successful transmissions happen by the end when the channel contention is low, while at the beginning the channel is severely stressed. 


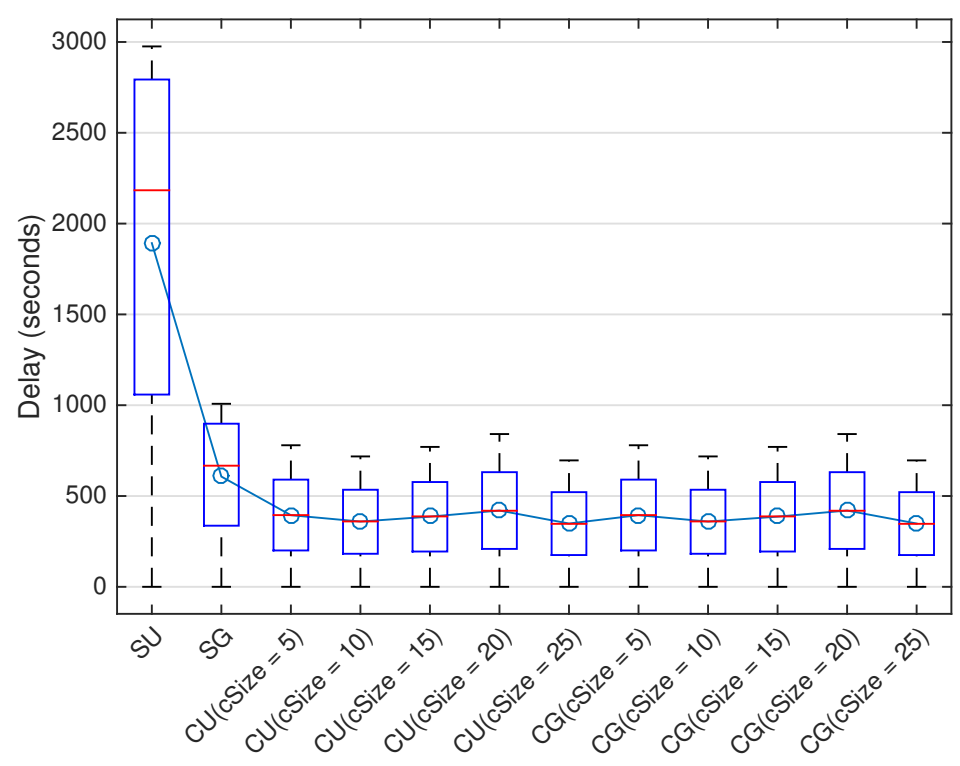

(a) Average Delay Per Packet

SU : Standard IEEE802.15.4 - Uniform Random Number Generator $S G$ : Standard IEEE802.15.4 - Geometric Random Number Generator $C U$ : Cluster-Centric MAC - Uniform Random Number Generator CG : Cluster-Centric MAC - Geometric Random Number Generator cSize : cluster size

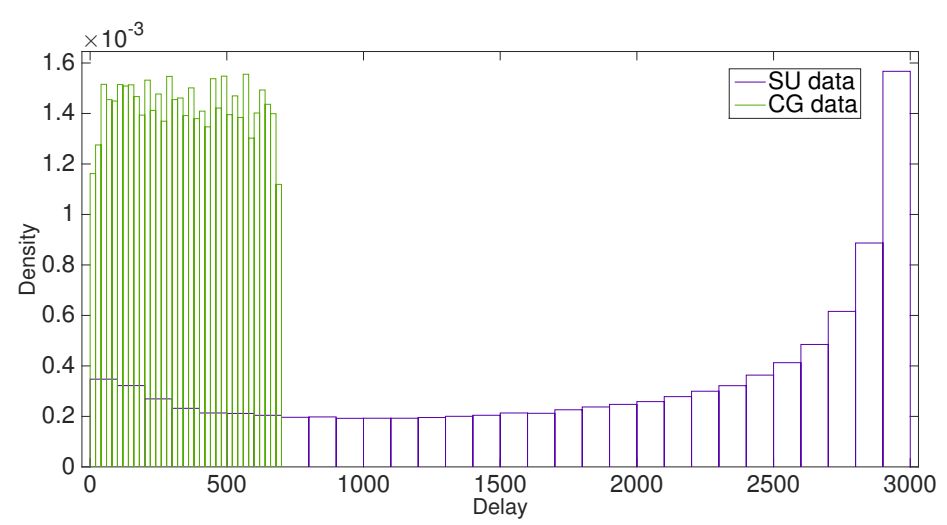

(b) Density Distribution

Figure 5.13: Delay Comparison $(\mathrm{N}=250)$ 


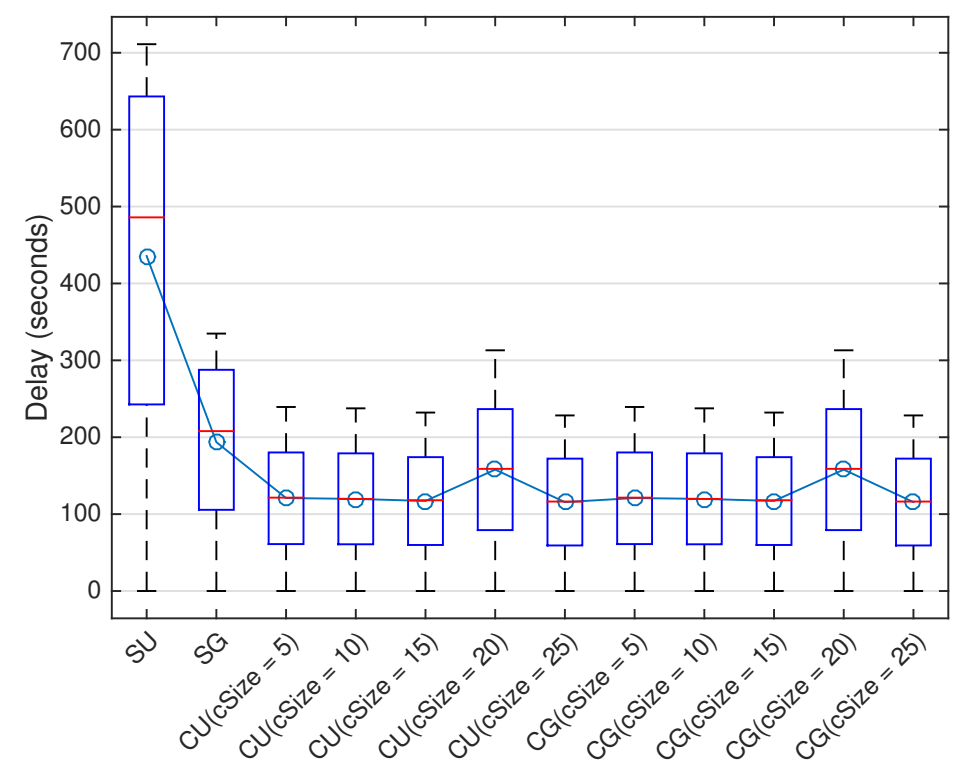

(a) Average Delay Per Packet

SU : Standard IEEE802.15.4 - Uniform Random Number Generator SG : Standard IEEE802.15.4 - Geometric Random Number Generator $C U$ : Cluster-Centric MAC - Uniform Random Number Generator CG : Cluster-Centric MAC - Geometric Random Number Generator cSize : cluster size

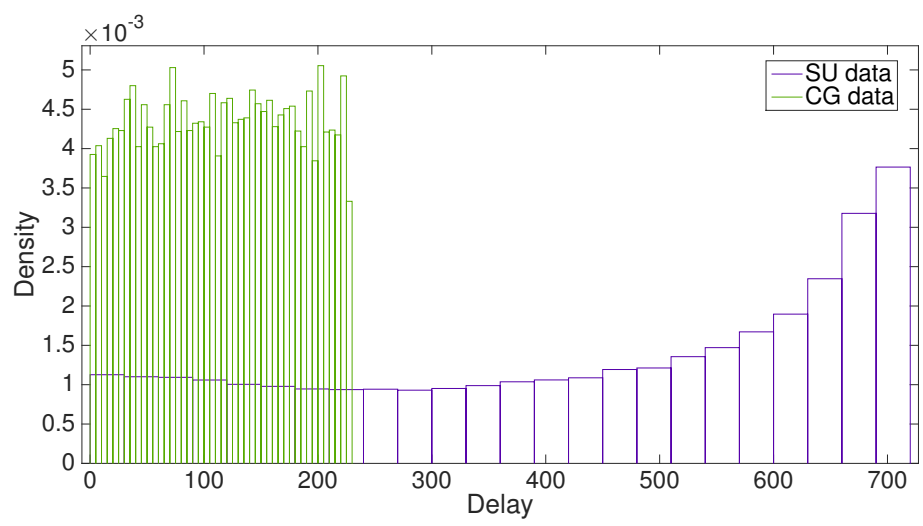

(b) Density Distribution

Figure 5.14: Delay Comparison $(\mathrm{N}=150)$ 


\subsubsection{Comparing Performance In Lossy Environment}

Failed packet delivery because of external noise is a common phenomena in wireless networks. A noisy network results in packet loss and only increases the number of packets in the network, as failed packets have to be retransmitted more often. Simulation parameters as shown in Table5.4 are used for evaluation.

To observe the performance of the proposed design under noisy conditions, the total time to transmit all network packets at fixed packet error probabilities is measured. Increased packet loss probability triggers higher retransmission rates, and a vicious cycle of successive retransmissions may develop due to the large number of nodes retransmitting simultaneously.

Figures 5.15a and 5.16a show that the standard IEEE802.15.4 MAC protocol suffers from high delays while the cluster-centric approach experiences a very gradual increase in time needed as the loss probability increases. This can be attributed to the fact that the proposed design reduces the overall throughput as shown previously, thus bringing down the network congestion and performing well even under lossy environment.

\begin{tabular}{|c|c|}
\hline Parameter & Value \\
\hline Network Size & 250 \\
\hline Cluster Size & 10 \\
\hline Data Packets Per Node & 1,215 \\
\hline Total Network Data Packets & 250,53750 \\
\hline Packet Error Probability & $0 \%, 5 \%, 10 \%, 15 \%, \ldots 90 \%$ \\
\hline MAC Algorithm & $\begin{array}{c}\text { Standard IEEE802.15.4 } \\
\text { Cluster-Centric MAC }\end{array}$ \\
\hline Random Number Generator & Uniform, Geometric \\
\hline
\end{tabular}

Table 5.4: Simulation Parameters For Performance Comparison In Lossy Environment 


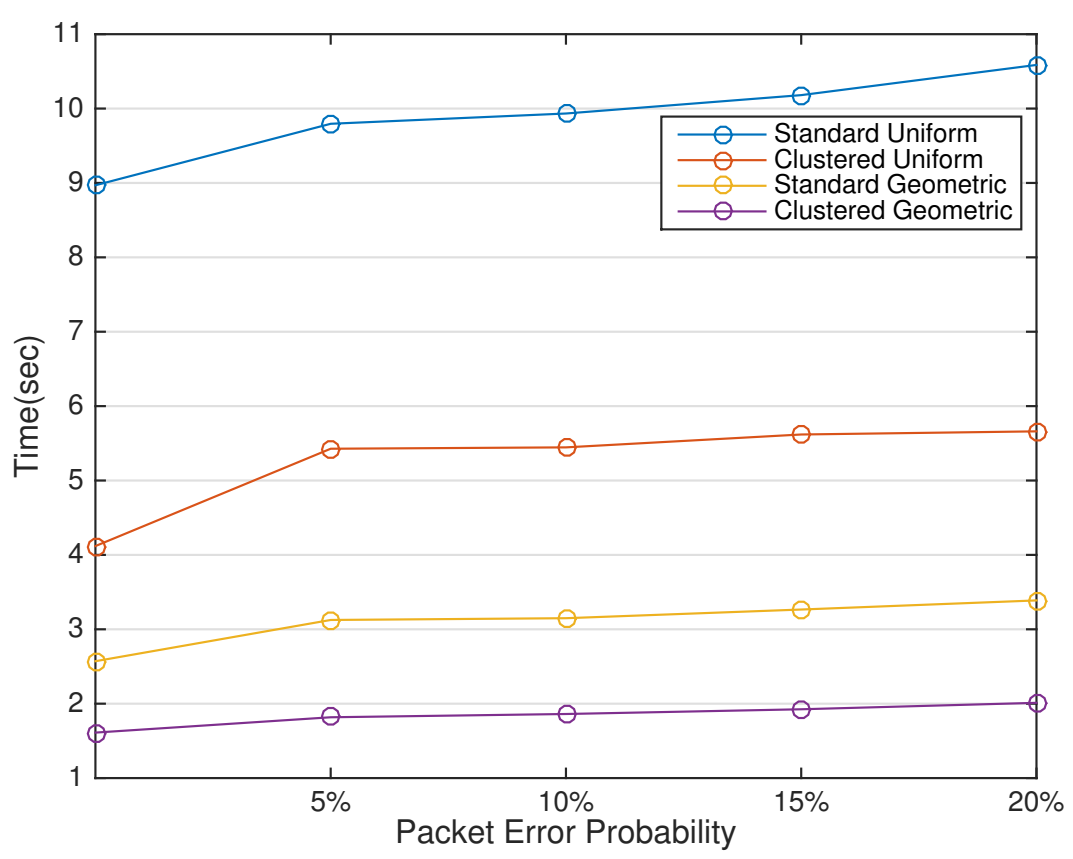

(a) Total Time To Transmit All Packets.

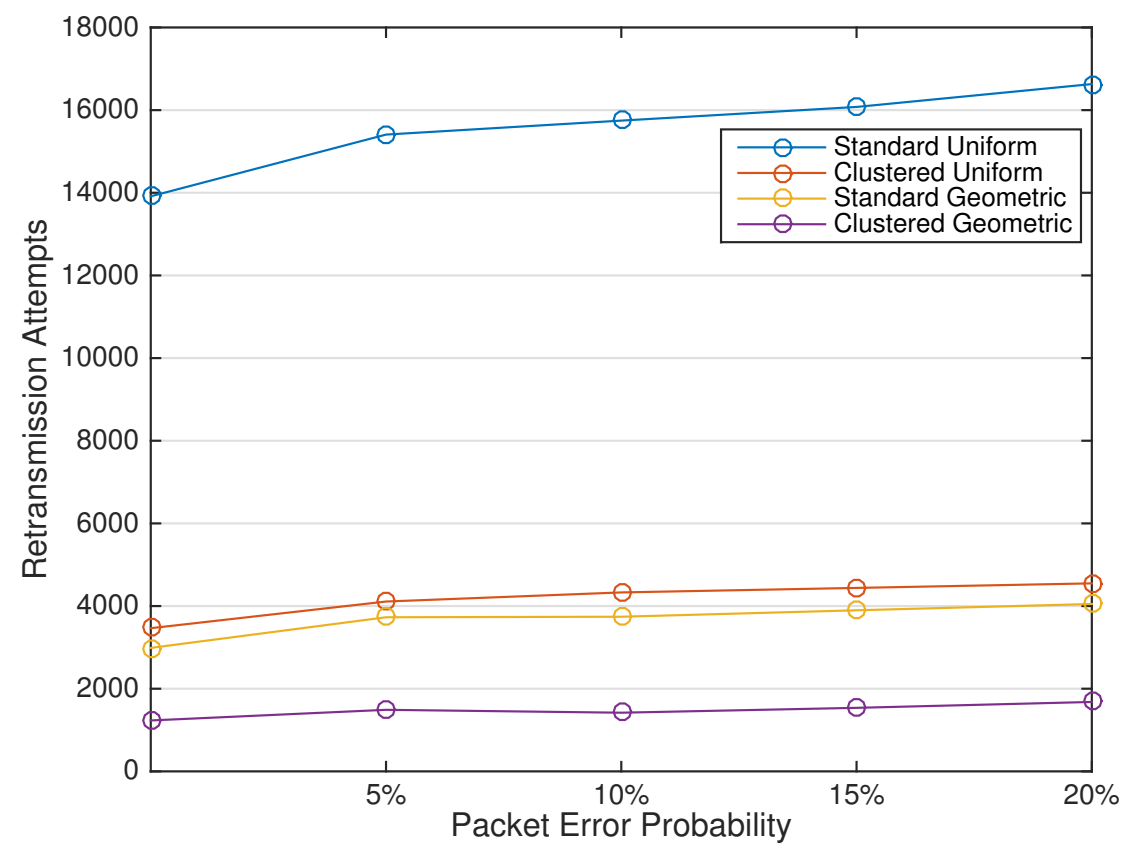

(b) Total Retransmission Attempts To Transmit All Packets.

Figure 5.15: Lossy Environment with Single Data Packet Per Node(N=250, $\mathrm{C}=10$ ) 


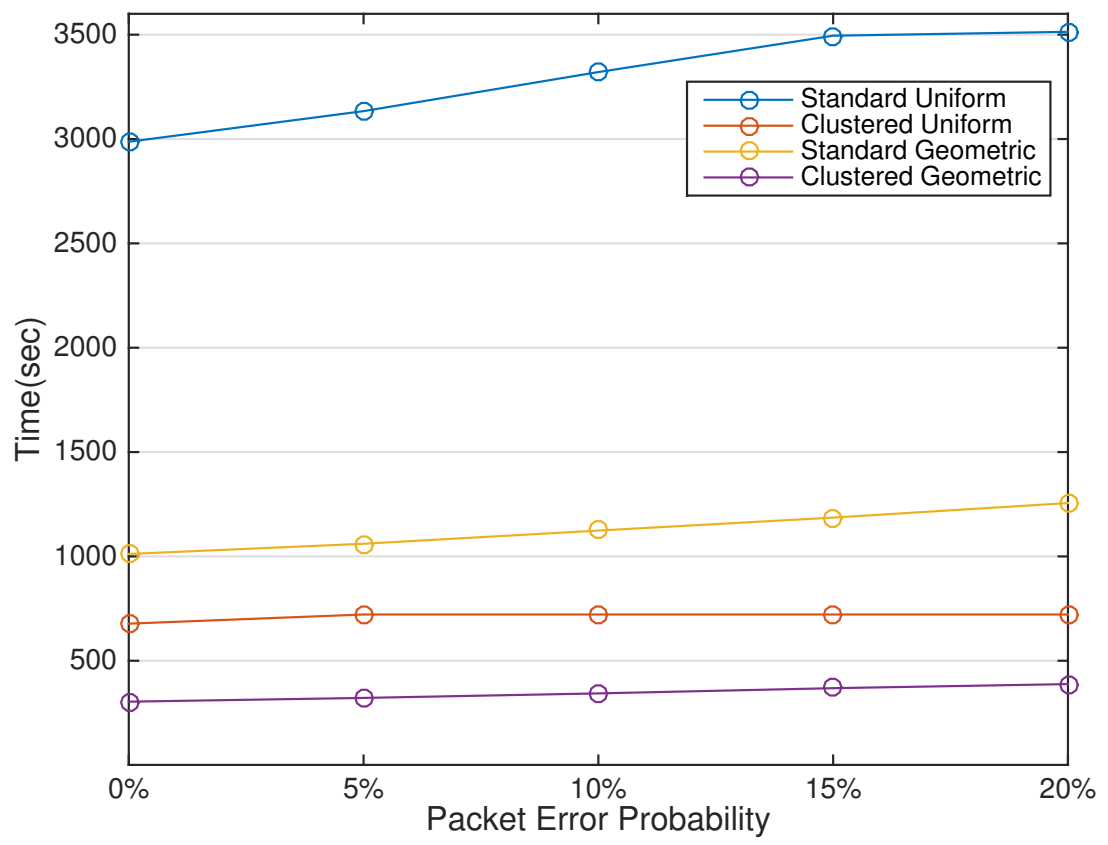

(a) Total Time To Transmit All Packets.

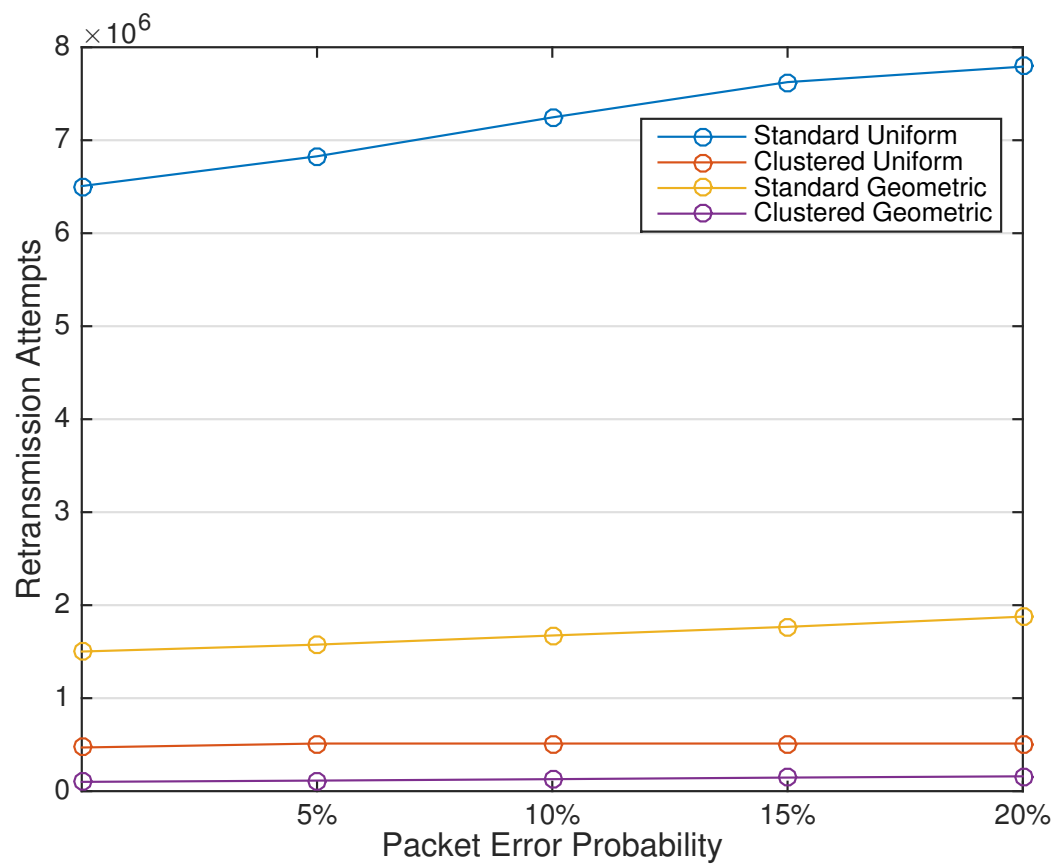

(b) Total Retransmission Attempts To Transmit All Packets.

Figure 5.16: Lossy Environment with Multiple Data Packets Per Node $(\mathrm{N}=250, \mathrm{C}=10)$ 
As expected the proposed design also fares well in terms of retransmission attempts owing to lower throughput as shown in Figures $5.15 \mathrm{~b}$ and 5.16b.

\section{Comparing The Worse and Best Performance under Lossy Environment}

The line plots in Figures 5.15 and 5.16 seem somewhat parallel, with the proposed scheme outperforming the standard scheme. However theoretically they should eventually meet at infinity with $100 \%$ packet loss probability. Which means that there should be a point when the worse performance of the proposed scheme matches the best performance of the standard scheme.

Figures 5.17 and 5.18 shows that even under a highly noisy channel the proposed scheme performs better than the standard scheme. Under the specified simulation conditions, the proposed scheme performs as good as the standard scheme in a noiseless channel, at as high as $65 \%$ and $80 \%$ packet error probabilities for network size 150 and 250 respectively.

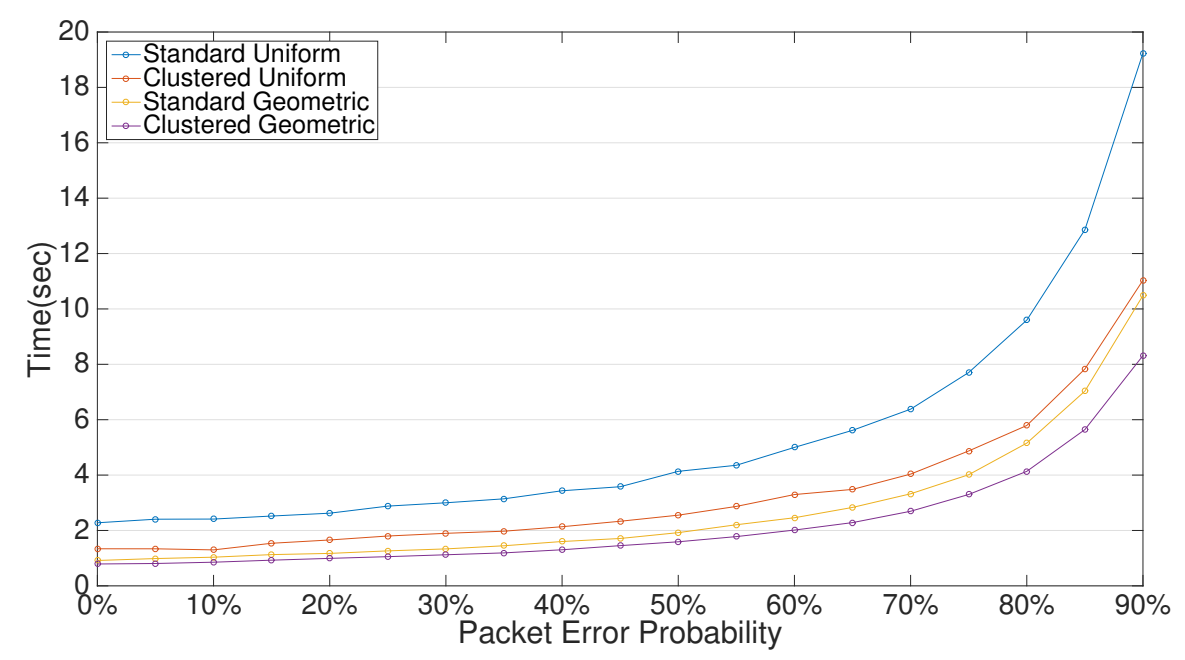

Figure 5.17: Lossy Network Performance Overlap (N=150) 


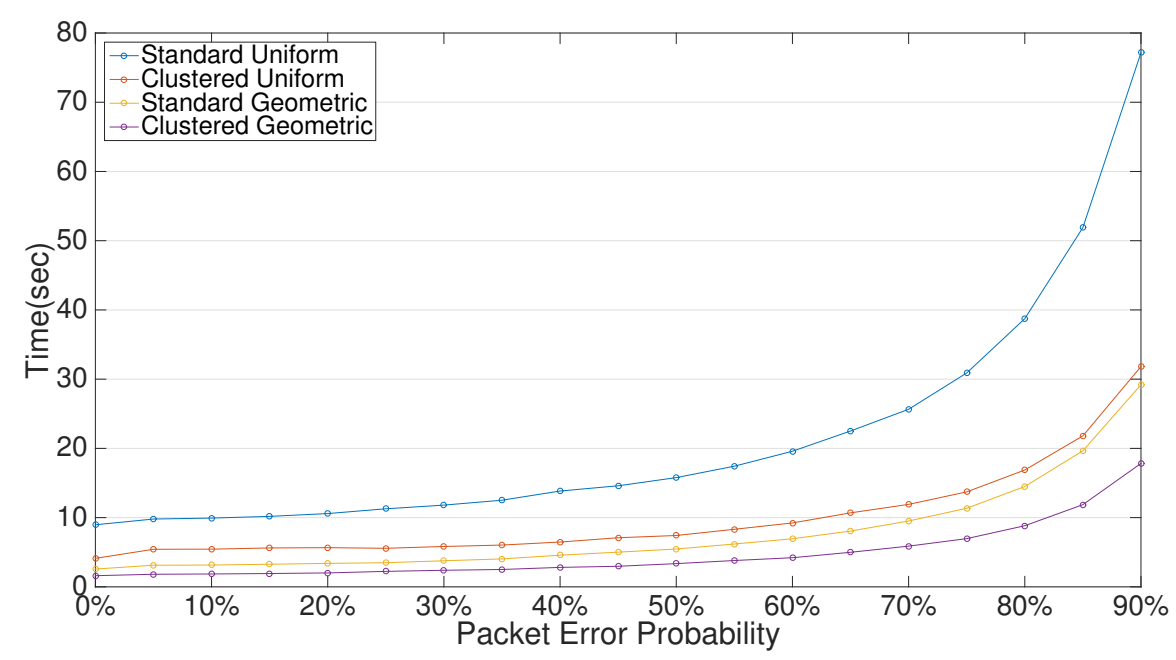

Figure 5.18: Lossy Network Performance Overlap ( $\mathrm{N}=250)$

\subsubsection{Comparing The Energy Consumption}

To put the energy consumption by the proposed design into perspective, simulations are run with the simulation parameters shown in Table 5.5, and the results are compared with Liu et al. [33] which is an example of a WSN approach designed to reduce energy consumption by performing in-

\begin{tabular}{|c|c|}
\hline Parameter & Value \\
\hline Network Size & 40 \\
\hline Cluster Size & 5 \\
\hline Samples Per Node & 50752 \\
\hline Samples Per Packet & $32,16,8,4,2,1$ \\
\hline Data Packets Per Node & $216,336,672,1344,2688,5376,10752$ \\
\hline Total Network Data Packets & $8640,13440,26880,53760$ \\
& $107520,215040,430080$ \\
\hline MAC Algorithm & Cluster-Centric MAC \\
\hline
\end{tabular}

Table 5.5: Simulation Parameters for energy consumption comparison 
network processing of SHM data. This approach has been selected, among others, as they have provided sufficient details on their evaluation and parameter values that enabled reasonable comparison; however, it should be noted that the comparison cannot be completely fair as their simulations did not take into account the MAC protocol's functionality. The values for comparison are extracted from the results shown in their paper.

A standard IEEE802.15.4 maximum payload capacity of 102 octets [80] can support up to 50 samples of 2 octets each. The proposed design is evaluated by using different sample sizes from one sample per packet (SPP) to the maximum possible 50 SPP. As per [33] a total of 10752 samples are assumed and energy values as specified in the paper are used. The simulations produced similar results for cluster sizes 5, 6, 7 and 8, so results for only cluster size 5 are cited. Since smaller SPP means higher number

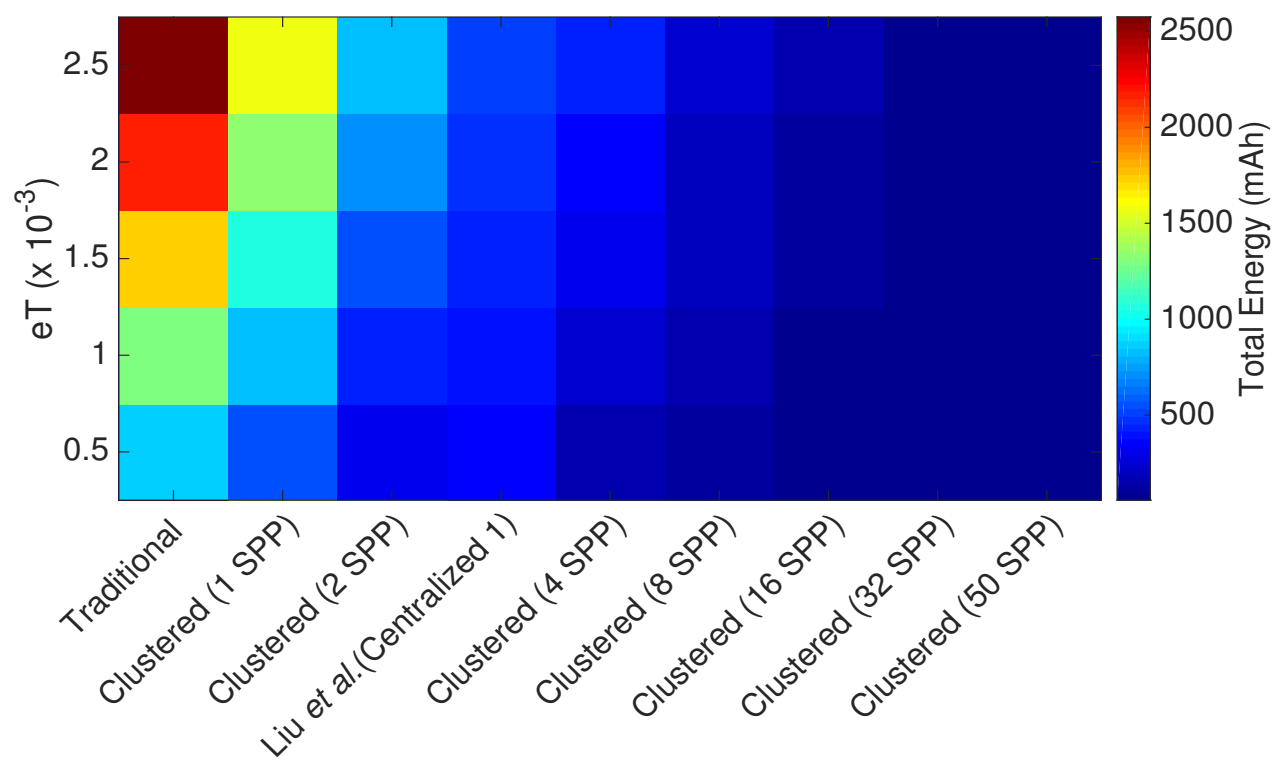

Figure 5.19: Energy Consumption Comparison With WSN For SHM Proposed By Liu et al.[33] Where eT (y-axis) Is The Transmission Power, Measured In mAh. 
of packets; intuitively it should mean that using 50 SPP not only results in lesser number of packets but also lesser contention, fewer retransmissions and lower energy consumption.

On the other hand, sending larger payloads require more energy than shorter payloads, but there are energy and throughput gains due to the amortization of the transmission overheads, and it has been shown that payload sizes of around 100 octets are near-optimal [81]. Simulation results as presented in Figure 5.19 also show that the proposed design is able to achieve better performance with as few as $4 \mathrm{SPP}$, without having to perform in-network processing.

The energy calculation has been described in Appendix A.

\subsubsection{Optimal Cluster Size}

Having established that the cluster-centric algorithm fares well against traditional approaches, the need to find the optimal cluster size arises. Simulations are run for all possible cluster sizes at increment of 1 from cluster size 2 to half of network size (i.e. when only 2 clusters are left) for network sizes 100, 150, 200, and 250. Results for only network size 150 and 250 will be discussed since all network sizes produced similar results. Simulation parameters as shown in Table 5.6 are used.

\begin{tabular}{|c|c|}
\hline Parameter & Value \\
\hline Network Size & 150,250 \\
\hline Cluster Size & 2 to half of network size \\
\hline Data Packets Per Node & 215 \\
\hline Total Network Data Packets & 53750 \\
\hline MAC Algorithm & Cluster-Centric MAC \\
\hline
\end{tabular}

Table 5.6: Simulation Parameters for Optimal Cluster Size 


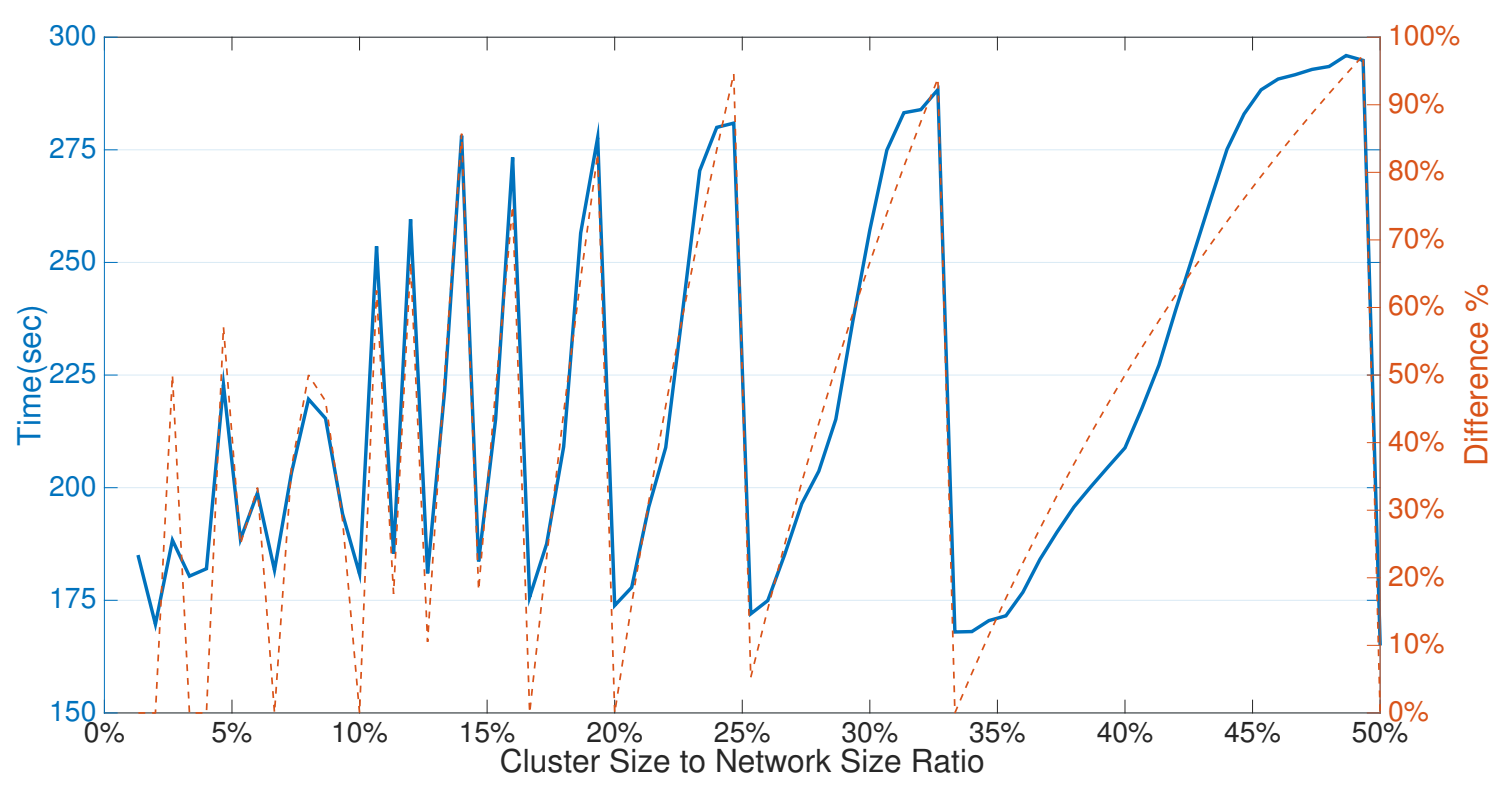

(a) Network Size 150

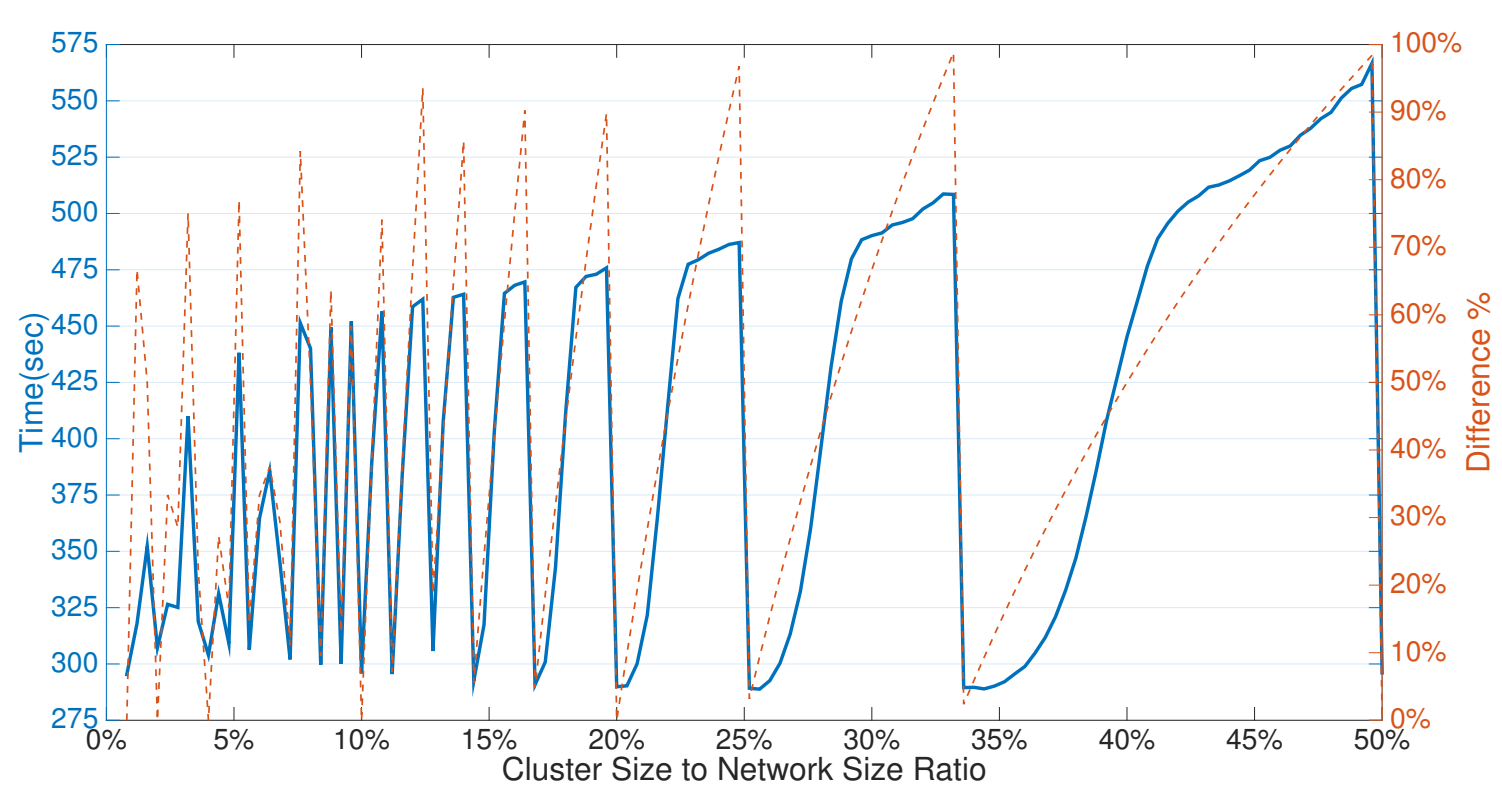

(b) Network Size 250

Figure 5.20: All Cluster Sizes 
The Time-axis in Figures 5.20 represents the total time taken by the network to successfully transmit all packets. If a network is divided into clusters of size $C$, then the number of nodes left to form the last cluster shall be between 1 to $C$. The Difference-axis represents the difference percentage between the number of nodes in the last cluster and the cluster size of the rest of the network. Both the y-axes represent a correlated repeating pattern, with better transmission times achieved when the difference percentage is lower, implying that equal cluster sizes produce best results.

\begin{tabular}{|c|c|}
\hline Parameter & Value \\
\hline Network Size & 250 \\
\hline Cluster Size & Unequal Cluster Sizes \\
\hline Number of Clusters & 10 \\
\hline Data Packets Per Node & 215 \\
\hline Total Network Data Packets & 53750 \\
\hline MAC Algorithm & Cluster-Centric MAC \\
\hline
\end{tabular}

Table 5.7: Simulation Parameters for Unequal Cluster Size

To further verify that equal cluster sizes indeed produce best results, simulations are run with parameters as shown in Table 5.7. Taking the assumption from previous result that equal cluster sizes should produce the best results, simulations are run for comparison against cluster size 25 . The x-axis in Figure 5.21 represents the maximum deviation the cluster sizes can have from 25 , and the Time-axis represents the total time taken by the network to successfully transmit all packets. The results in Figure 5.21 show that the optimal results are indeed produced by equal cluster sizes. 


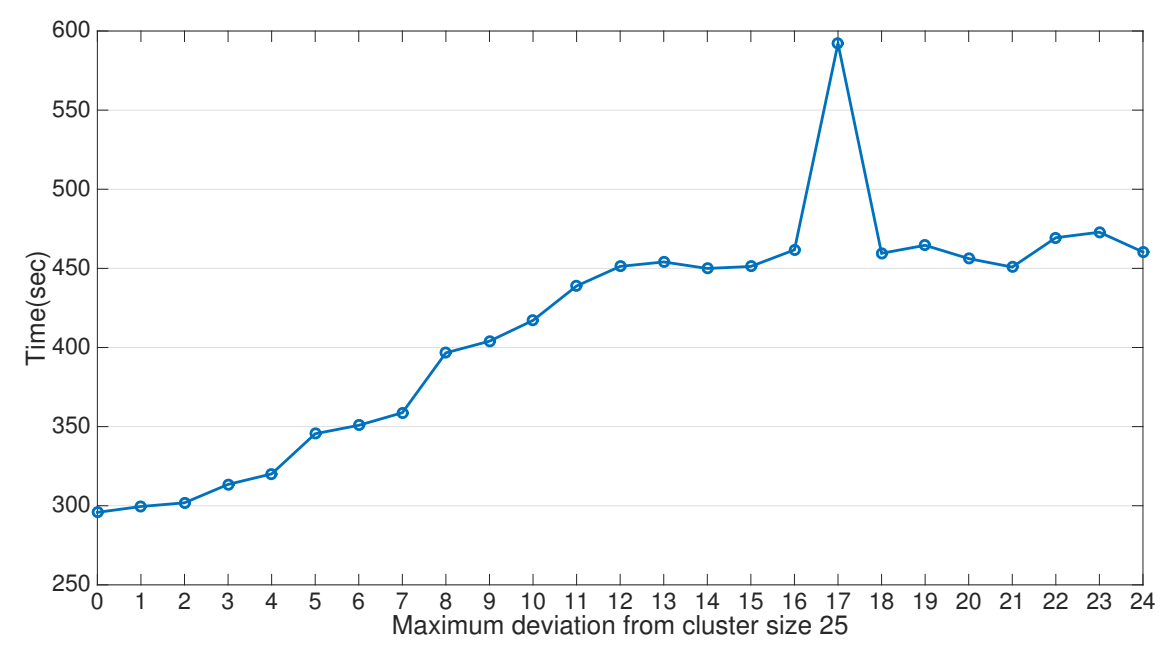

Figure 5.21: One Set Of Cluster Sizes (N=250)

\section{Optimal Cluster Count}

The result analysis proves that equal cluster sizes produce the best transmission times. However, a given network can have multiple possible cluster sizes that partition it equally. This motivates the search for optimal cluster count, such that the difference between the cluster sizes is no more than 1.

Simulations are run by first dividing the network into fixed number of empty clusters. Then one node is assigned to each cluster, till every cluster has one node each. This is repeated until no nodes are left to be assigned to clusters, ensuring that the difference between the cluster sizes is no more than one. Simulation parameters as shown in Table 5.8 are used for evaluation.

The results obtained are shown in Figure 5.22. The Time-axis represents the total time taken by the network to successfully transmit all data packets. The red colour stems represent the points where cluster sizes are equal. 


\begin{tabular}{|c|c|}
\hline Parameter & Value \\
\hline Network Size & $100,150,200,250,300$ \\
\hline Number of Clusters & 2 to half of network size \\
\hline Data Packets Per Node & 215 \\
\hline Total Network Data Packets & $21500,32250,43000,53750,64500$ \\
\hline MAC Algorithm & Cluster-Centric MAC \\
\hline
\end{tabular}

Table 5.8: Simulation Parameters for Optimal Cluster Count

It can be observed from Figure 5.22 that lower cluster count produce better transmission times, which can be attributed to the fact that when a node is successfully able to transmit its data packet, a big chunk of network (neighbour nodes) goes out of contention. That is each successful transmission results in big drop in contention, hence faster overall network transmission.

Results however also show that too large cluster sizes are also not optimal. While having 2 clusters give the best performance for smaller network sizes like 100 and 150, for larger network size it leads to too big clusters. The reason large cluster sizes do not work is because even when there is only one cluster left to transmit in a given round, there is still a significant network contention because of large number nodes in the cluster.

As the cluster count approaches half of the network size, it can be noticed that the transmission time starts to drop. This decreasing pattern of transmission time continues longer for larger network sizes compared to smaller networks. Even though smaller cluster sizes result in slower decrease in contention per successful transmission, the resulting low contention hangs for a long time because of large number of candidate clusters left to transmit. This pattern is more apparent in larger network sizes, as they can have more number of clusters. 


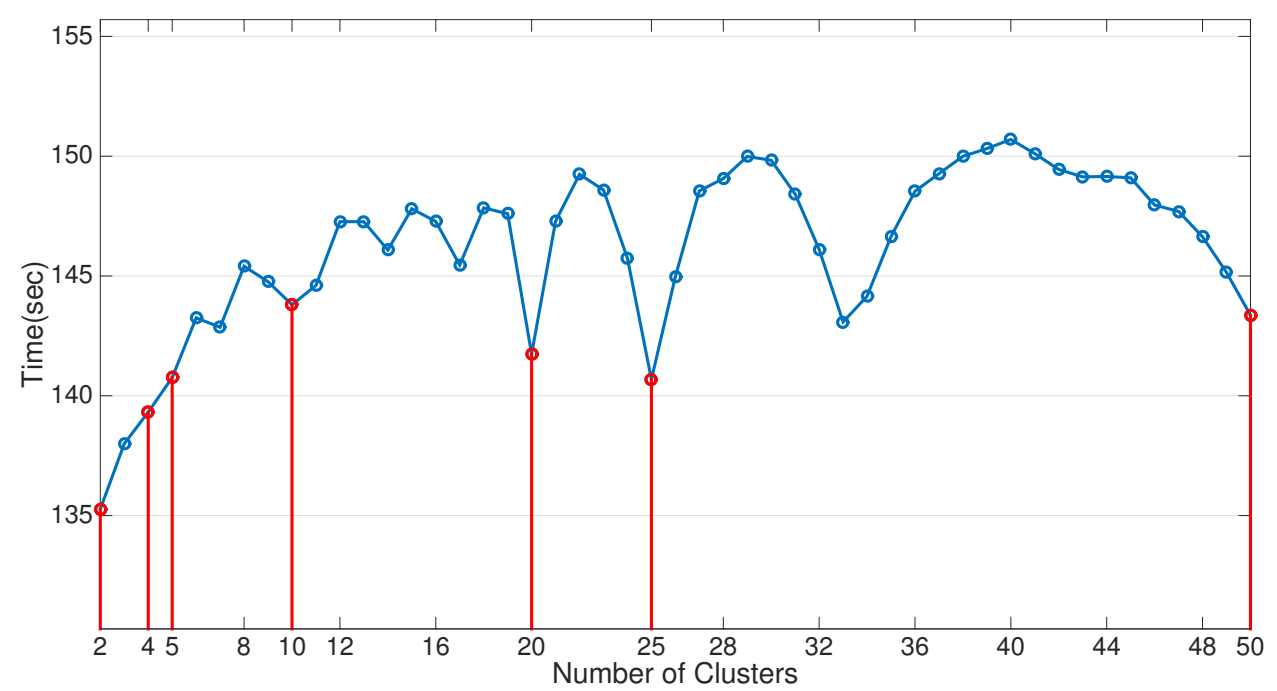

(a) Network Size 100

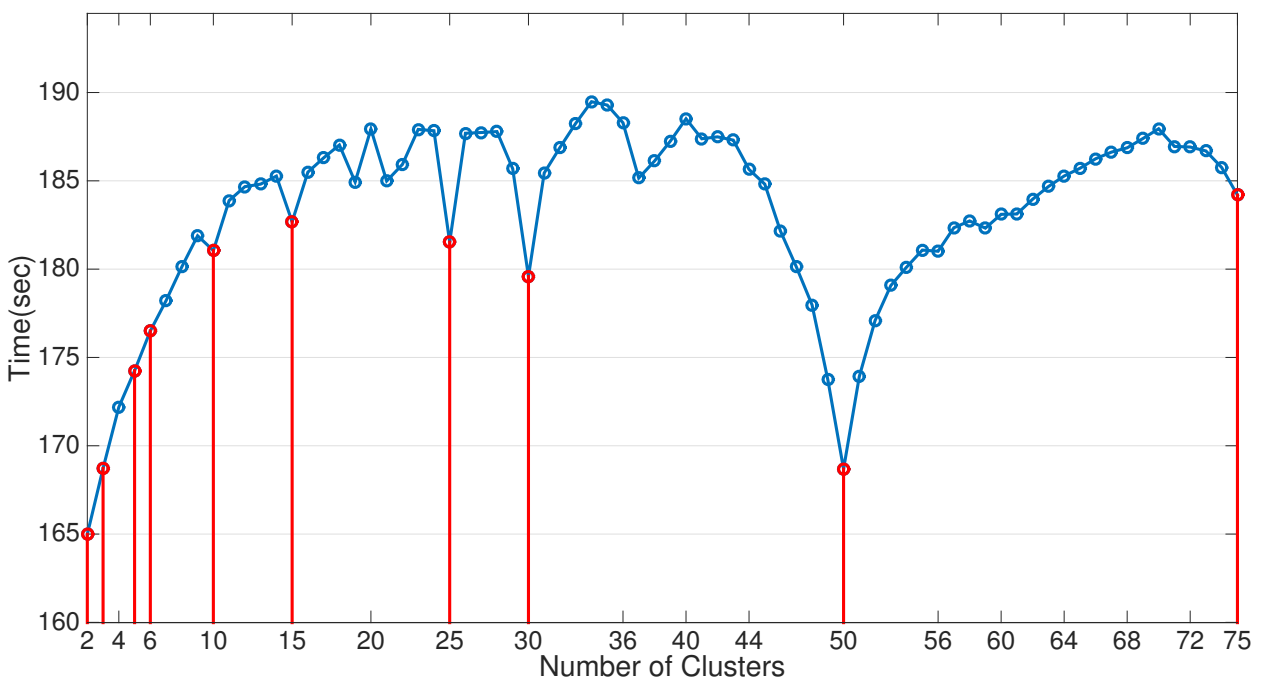

(b) Network Size 150

Figure 5.22: All Cluster Count $(\mathrm{N}=100,150)$ 


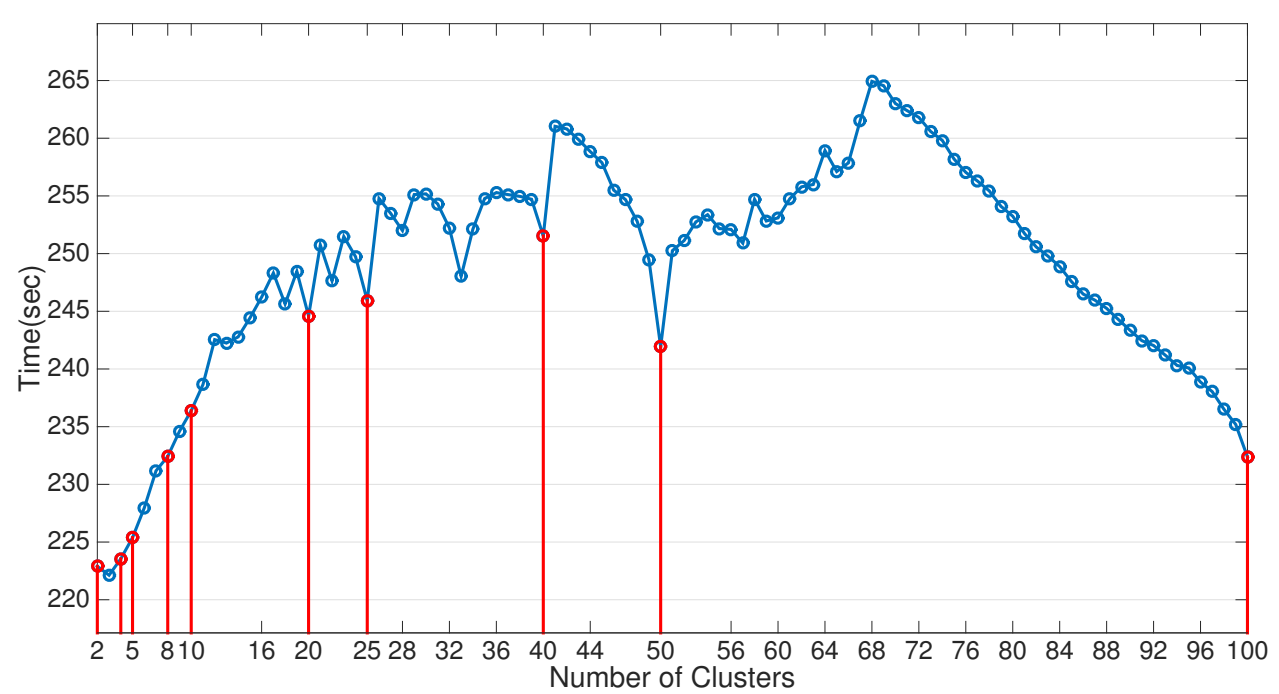

(a) Network Size 200

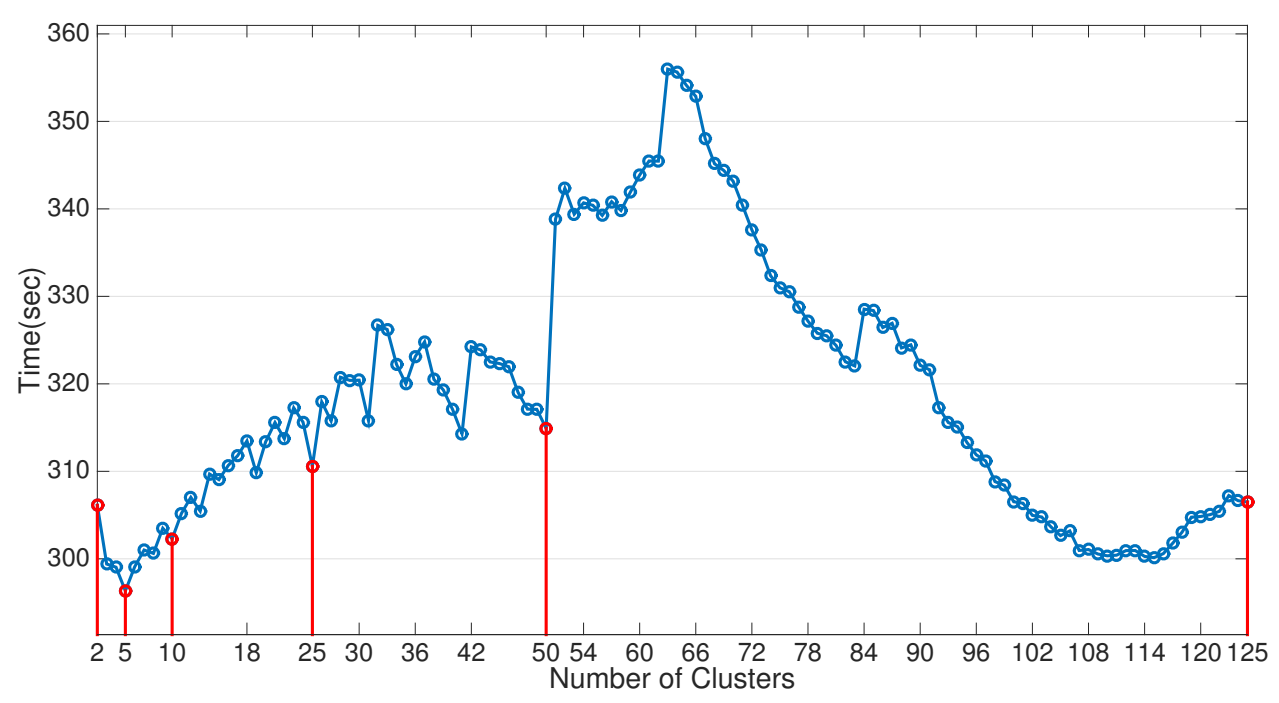

(b) Network Size 250

Figure 5.23: All Cluster Count (N=200, 250) 


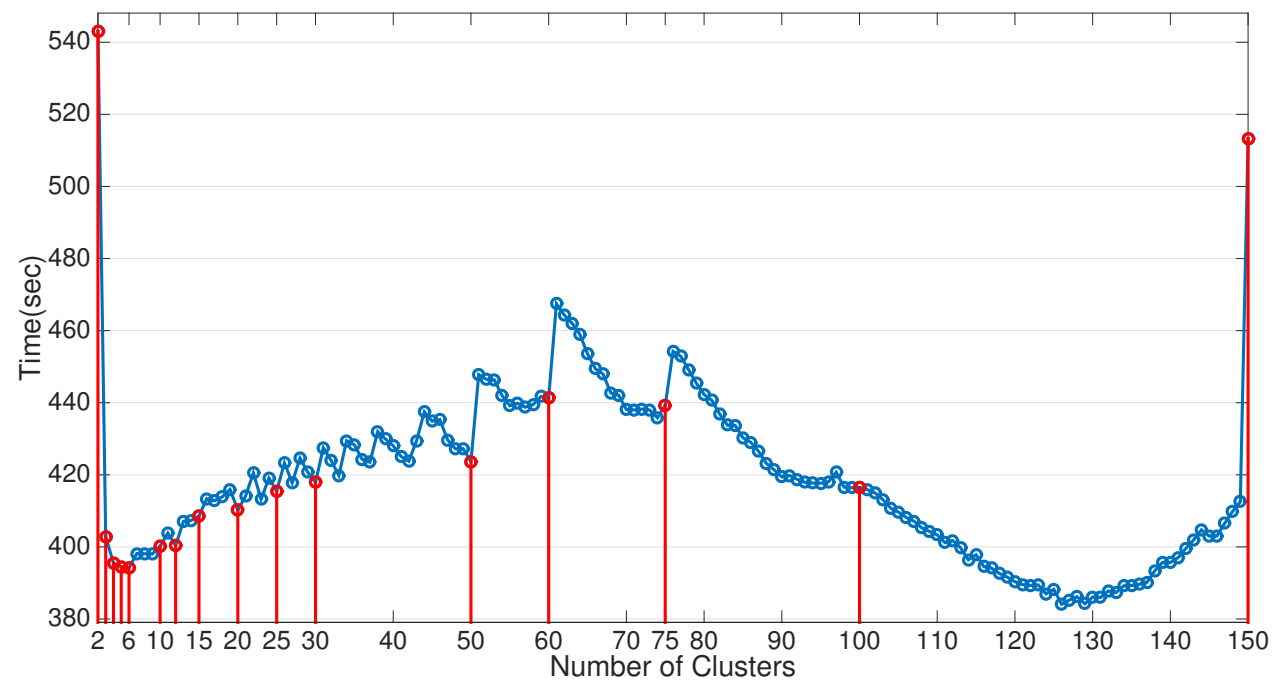

Figure 5.24: All Cluster Count $(\mathrm{N}=300)$

It is also worth noticing in the results, that the data points with equal cluster sizes have on an average better performance compared to other cluster size combinations. Also the worst performances are observed when all the cluster sizes are not equal and the number of clusters is close to onefourth of the network size.

To verify the above assertions made with respect to optimal cluster count, simulations are run for an even larger network. Figure 5.24 shows the results for network size 300 . It can be observed that all the above identified assertions are scalable and hold true for a larger network. However if there are too many clusters, it takes the network longer to reach lower contention. The time taken to reach lower contention, outweighs the transmission time benefits obtained during low contention.

From SHM perspective, it is not practical to have just two clusters. Neither does having a lot of clusters with just two nodes each make sense. Also the inherent assumption of this thesis that a cluster should have spatially correlated data, cannot be met with either just two clusters or clusters consisting of two nodes each. However based on observation, a structural 
health engineer is advised to not only keep the cluster sizes equal but also stay away from keeping the number of clusters close to one-fourth of the network size.

\subsection{Summary}

The result evaluation proves that under bursty network conditions the proposed design performs better than the traditional MAC algorithms and brings about the following improvements:

1. Network bias is eliminated and channel is allocated fairly among nodes.

2. Network congestion is reduced by reducing the network throughput.

3. Reduced congestion leads to reduced delay, transmission times, and retransmission attempts, and increased goodput.

4. Reduced retransmission attempts lead to reduced energy usage.

5. The above performance improvements lead to a scalable network that performs well even under lossy environment.

The proposed design is proven to give better performance with equal cluster sizes, and deteriorates gradually with increasing inter-cluster size difference. 


\section{Chapter 6}

\section{Conclusion}

This thesis discussed the different existing approaches to the use of smart WSNs in SHM. Much of the work that has been done focused on determining the optimal network structure, viz. clustering of sensors, to facilitate in-network distributed processing of SHM data for modal analysis. The aim of these existing approaches is to reduce the bandwidth requirements imposed by voluminous raw SHM data on the network, and exploit the computational power of sensors to perform distributed computation (modal analysis) that is usually done at the data acquisition centre (or PAN coordinator).

Clustering in WSNs has been extensively studied from the networking perspective with the aim of achieving optimal network performance without consideration to the content of the data being delivered. This thesis looks at clustering from a new perspective and utilizes clustering in a novel way to address an unstudied problem of bias towards nodes that are closer to the PAN coordinator.

The proposed cluster-centric MAC protocol treats each cluster as a supernode when arbitrating access to the wireless channel, and has been shown to significantly reduce contention leading to improved overall network performance. 


\section{Contributions}

The main contribution of this thesis is a cluster-centric MAC protocol that answers problems much prevalent with the WSNs in SHM. To reiterate, the proposed design makes the following improvements over the standard IEEE802.15.4 protocol:

- The cluster bias induced due to proximity to the sink node is eliminated.

- Network congestion is reduced by decreasing the network throughput.

- Transmission delay, transmission time, retransmission attempts, and energy usage are decreased due to the reduced congestion. This also results in increased goodput.

- Network scalability and harsh network condition tolerance is also achieved due to the above stated improvements.

\section{Results}

The direct benefits of the proposed design can be summarised as follows:

- Unbiased performance in star-topology based bursty sensor networks.

- Energy efficient performance.

- Faster transmission times even in highly congested networks.

- The simple nature of the proposed design appeals to the universality of its possible application in other (non IEEE802.15.4) bursty wireless networks as well. 


\section{Limitations and Future Work}

This thesis presents a new strand of possible research with the presence of network bias much prevalent in WSNs, and a new cluster-centric design is proposed along the way. The scope of this thesis is however limited and the following future work is suggested to fill the gap:

- The thesis limits itself to only simulation based evaluation, opening up opportunities for theoretical and test-bed based evaluation of the proposed design in future.

- The proposed design assumes that the clusters are manually partitioned by the structural engineers. Future work could explore the possibility of dynamic autonomous clustering.

- The evaluation section assumes that a fixed amount of data is generated at each node, a real SHM system however might generate variable data. The performance of the proposed design should be studied with variable amount of data as well.

- Energy conservation is a big concern with WSNs and evergy harvesting WSNs is an area of active research. The proposed model and evaluation limit itself to a simple battery model, but future work could extend the research to energy harvesting. 


\section{Appendix A}

\section{Energy Consumption Calculation}

Table A. 1 shows the values used for energy consumption comparison with Liu et al.[33] as stated in [33].

\begin{tabular}{|c|c|c|}
\hline Variable & & Value \\
\hline Total Number of Samples & $-N$ & 10752 \\
\hline Sampling Energy Cost & $-e_{S}(\mathrm{mAh})$ & $1.1 e-4$ \\
\hline Receiving Energy Cost & $-e_{R}(\mathrm{mAh})$ & $5 e-4$ \\
\hline Transmission Energy Cost & $-e_{T}\left(\times 10^{-3} \mathrm{mAh}\right)$ & $0.5,1,1.5,2,2.5$ \\
\hline
\end{tabular}

Table A.1: Values Used For Energy Consumption Calculation

Using these values the total energy used by a node $(n)$ is:

$$
e_{n}=R_{x} \times e_{R}+T_{x} \times e_{T}+N \times e_{S}
$$

Where $R_{x}$ and $T_{x}$ are the total number of packets received and transmitted by a node.

Which means that for a network of 40 nodes, the total energy consumed by the whole network is,

$$
\sum_{n=1}^{40} e_{n}
$$




\section{Bibliography}

[1] Committee on Networked Systems of Embedded Computers, National Research Council, Embedded Everywhere: A Research Agenda for Networked Systems of Embedded Computers. National Academies Press, 2001.

[2] T. Bokareva, W. Hu, S. Kanhere, B. Ristic, N. Gordon, T. Bessell, M. Rutten, and S. Jha, "Wireless sensor networks for battlefield surveillance," in Proceedings of the land warfare conference, pp. 1-8, 2006.

[3] A. Mainwaring, D. Culler, J. Polastre, R. Szewczyk, and J. Anderson, "Wireless Sensor Networks for Habitat Monitoring," in Proceedings of the 1st ACM International Workshop on Wireless Sensor Networks and Applications, WSNA '02, (New York, NY, USA), pp. 88-97, ACM, 2002.

[4] K. Chintalapudi, T. Fu, J. Paek, N. Kothari, S. Rangwala, J. Caffrey, R. Govindan, E. Johnson, and S. Masri, "Monitoring civil structures with a wireless sensor network," Internet Computing, IEEE, vol. 10, pp. 26-34, March 2006.

[5] C.-T. Ng and T. H. Chan, "Special Issue on Structural Health Monitoring of Civil Structures," Structural Health Monitoring, vol. 13, no. 4, pp. 345-346, 2014.

[6] J. P. Lynch and K. J. Loh, "A Summary Review of Wireless Sensors and Sensor Networks for Structural Health Monitoring," Shock and Vibration Digest, vol. 38, pp. 91-128, Mar 2006. 
[7] M. W. Lin, A. O. Abatan, and Y. Zhou, “Transverse shear response monitoring of concrete cylinder using embedded high-sensitivity ETDR sensor," vol. 3988, pp. 319-328, 2000.

[8] M. Çelebi, "Seismic Instrumentation of Buildings (With Emphasis on Federal Buildings)," Tech. Rep. 0-7460-68170, 2002.

[9] C. Farrar, "Historical Overview of Structural Health Monitoring," Lecture Notes on Structural Health Monitoring Using Statistical Pattern Recognition, 2001.

[10] B. Li, Z. Sun, K. Mechitov, G. Hackmann, C. Lu, S. Dyke, G. Agha, and B. Spencer, "Realistic case studies of wireless structural control," in Cyber-Physical Systems (ICCPS), 2013 ACM/IEEE International Conference on, pp. 179-188, April 2013.

[11] J. M. Caicedo, J. Marulanda, P. Thomson, and S. J. Dyke, "Monitoring of bridges to detect changes in structural health," in American Control Conference, 2001. Proceedings of the 2001, vol. 1, pp. 453-458, IEEE, 2001.

[12] H. Bai, M. Atiquzzaman, and D. Lilja, "Wireless sensor network for aircraft health monitoring," in Proceedings of the First International Conference on Broadband Networks, 2004, BroadNets 2004., pp. 748-750, Oct 2004.

[13] T. Chan, L. Yu, H. Tam, Y. Ni, S. Liu, W. Chung, and L. Cheng, "Fiber Bragg grating sensors for structural health monitoring of Tsing Ma bridge: Background and experimental observation," Engineering Structures, vol. 28, no. 5, pp. 648-659, 2006.

[14] M. I. Todorovska and M. D. Trifunac, "Earthquake damage detection in the Imperial County Services Building II: Analysis of novelties via wavelets," Structural Control and Health Monitoring, vol. 17, pp. 895917, 122010. 
[15] M. Cardei and D.-Z. Du, "Improving wireless sensor network lifetime through power aware organization," Wireless Networks, vol. 11, no. 3, pp. 333-340, 2005.

[16] J. Polastre, J. Hill, and D. Culler, "Versatile Low Power Media Access for Wireless Sensor Networks," in Proceedings of the 2nd International Conference on Embedded Networked Sensor Systems, SenSys '04, (New York, NY, USA), pp. 95-107, ACM, 2004.

[17] Y.-W. Hong, W.-J. Huang, F.-H. Chiu, and C.-C. Kuo, "Cooperative Communications in Resource-Constrained Wireless Networks," Signal Processing Magazine, IEEE, vol. 24, pp. 47-57, May 2007.

[18] W. K. G. Seah, Z. A. Eu, and H. Tan, "Wireless sensor networks powered by ambient energy harvesting (WSN-HEAP) - Survey and challenges," in Proceedings of the 1st International Conference on Wireless Communication, Vehicular Technology, Information Theory and Aerospace Electronic Systems Technology (Wireless VITAE), (Aalbory, Denmark), 17-20 May 2009.

[19] Y. Sun, S. Du, O. Gurewitz, and D. B. Johnson, “DW-MAC: A Low Latency, Energy Efficient Demand-wakeup MAC Protocol for Wireless Sensor Networks," in Proceedings of the 9th ACM International Symposium on Mobile Ad Hoc Networking and Computing, MobiHoc '08, (New York, NY, USA), pp. 53-62, ACM, 2008.

[20] W. Ye, J. Heidemann, and D. Estrin, "An energy-efficient mac protocol for wireless sensor networks," in INFOCOM 2002. Twenty-First Annual Joint Conference of the IEEE Computer and Communications Societies. Proceedings. IEEE, vol. 3, pp. 1567-1576, IEEE, 2002.

[21] T. van Dam and K. Langendoen, "An Adaptive Energy-efficient MAC Protocol for Wireless Sensor Networks," in Proceedings of the 1st Inter- 
national Conference on Embedded Networked Sensor Systems, SenSys '03, (New York, NY, USA), pp. 171-180, ACM, 2003.

[22] IEEE Std 802.15.4-2006, Wireless Medium Access Control (MAC) and Physical Layer (PHY) Specifications for Low-Rate Wireless Personal Area Networks (WPANs). IEEE, 2006.

[23] W. Ye, J. Heidemann, and D. Estrin, "Medium access control with coordinated adaptive sleeping for wireless sensor networks," Networking, IEEE/ACM Transactions on, vol. 12, pp. 493-506, June 2004.

[24] L. Kleinrock and F. Tobagi, "Packet Switching in Radio Channels: Part I-Carrier Sense Multiple-Access Modes and Their ThroughputDelay Characteristics," IEEE Transactions on Communications, vol. 23, pp. 1400-1416, Dec 1975.

[25] S. Kim, S. Pakzad, D. Culler, J. Demmel, G. Fenves, S. Glaser, and M. Turon, "Health Monitoring of Civil Infrastructures Using Wireless Sensor Networks," in Information Processing in Sensor Networks, 2007. IPSN 2007. 6th International Symposium on, pp. 254-263, April 2007.

[26] N. Xu, S. Rangwala, K. K. Chintalapudi, D. Ganesan, A. Broad, R. Govindan, and D. Estrin, "A Wireless Sensor Network For Structural Monitoring," in Proceedings of the 2nd International Conference on Embedded Networked Sensor Systems, SenSys '04, (New York, NY, USA), pp. 13-24, ACM, 2004.

[27] Y. Tselishchev and A. Boulis, "Wireless Sensor Network tesbed for structural Health Monitoring of bridges," in Sensors, 2009 IEEE, pp. 1796-1799, Oct 2009.

[28] K. Leentvaar and J. Flint, "The Capture Effect in FM Receivers," IEEE Transactions on Communications, vol. 24, pp. 531-539, May 1976. 
[29] O. Younis and S. Fahmy, "HEED: a hybrid, energy-efficient, distributed clustering approach for ad hoc sensor networks," Mobile Computing, IEEE Transactions on, vol. 3, pp. 366-379, Oct 2004.

[30] M. M. Afsar and M.-H. Tayarani-N, "Clustering in sensor networks: A literature survey," Journal of Network and Computer Applications, vol. 46, pp. 198-226, 2014.

[31] W. R. Heinzelman, A. Chandrakasan, and H. Balakrishnan, "Energyefficient communication protocol for wireless microsensor networks," in System sciences, 2000. Proceedings of the 33rd annual Hawaii international conference on, pp. 10-pp, IEEE, Jan 2000.

[32] A. T. Zimmerman, M. Shiraishi, R. A. Swartz, and J. P. Lynch, "Automated modal parameter estimation by parallel processing within wireless monitoring systems," Journal of Infrastructure Systems, vol. 14, no. 1, pp. 102-113, 2008.

[33] X. Liu, J. Cao, S. Lai, C. Yang, H. Wu, and Y. L. Xu, "Energy efficient clustering for WSN-based structural health monitoring," in Proceedings of the 30th IEEE International Conference on Computer Communications (INFOCOM), (Shanghai, China), pp. 2768-2776, 10-15 April 2011.

[34] A. Jindal and M. Liu, "Networked Computing in Wireless Sensor Networks for Structural Health Monitoring," IEEE Transactions on Networking, vol. 20, pp. 1203-1216, August 2012.

[35] G. Hackmann, W. Guo, G. Yan, Z. Sun, C. Lu, and S. Dyke, "Cyberphysical codesign of distributed structural health monitoring with wireless sensor networks," IEEE Transactions on Parallel and Distributed Systems, vol. 25, no. 1, pp. 63-72, 2014.

[36] Saurabh Singh, Winston K.G. Seah, and Bryan Ng, "Cluster-Centric Medium Access Control for WSNs in Structural Health Monitoring," 
in 13th International Symposium on Modeling and Optimization in Mobile, Ad Hoc, and Wireless Networks (WiOpt), (Mumbai, India), May 2015.

[37] E. Straser, A. Kiremidjian, T. Meng, and L. Redlefsen, "A Modular, Wireless Network Platform for Monitoring Structures," in Proceedings of the 16th International Modal Analysis Conference, vol. 3243, p. 450, 1998.

[38] H. Sohn, C. R. Farrar, F. M. Hemez, D. D. Shunk, D. W. Stinemates, B. R. Nadler, and J. J. Czarnecki, A review of structural health monitoring literature: 1996-2001. Los Alamos National Laboratory Los Alamos, NM, 2004.

[39] A. Basharat, N. Catbas, and M. Shah, "A framework for intelligent sensor network with video camera for structural health monitoring of bridges," in Pervasive Computing and Communications Workshops, 2005. PerCom 2005 Workshops. Third IEEE International Conference on, pp. 385-389, March 2005.

[40] A. Cunha and E. Caetano, "Experimental modal analysis of civil engineering structures," Journal of Sound and Vibration, vol. 40, June 2006.

[41] P. Andersen, R. Brincker, M. Goursat, and L. Mevel, “Automated modal parameter estimation for operational modal analysis of large systems," in International Operational Modal Analysis Conference (IOMAC), pp. 299-308, 2007.

[42] O. Salawu, "Detection of structural damage through changes in frequency: a review," Engineering Structures, vol. 19, no. 9, pp. 718-723, 1997.

[43] J.-T. Kim, Y.-S. Ryu, H.-M. Cho, and N. Stubbs, "Damage identification in beam-type structures: frequency-based method vs mode- 
shape-based method," Engineering structures, vol. 25, no. 1, pp. 57-67, 2003.

[44] S. W. Doebling, C. R. Farrar, M. B. Prime, and D. W. Shevitz, "Damage identification and health monitoring of structural and mechanical systems from changes in their vibration characteristics: a literature review," tech. rep., Los Alamos National Lab., NM (United States), 1996.

[45] G. Anastasi, M. Conti, M. Di Francesco, and A. Passarella, "Energy conservation in wireless sensor networks: A survey," Ad hoc networks, vol. 7, no. 3, pp. 537-568, 2009.

[46] B. Aygün and V. C. Gungor, "Wireless sensor networks for structure health monitoring: recent advances and future research directions," Sensor Review, vol. 31, no. 3, pp. 261-276, 2011.

[47] J. Caffrey, R. Govindan, E. Johnson, B. Krishnamachari, S. Masri, G. Sukhatme, K. Chintalapudi, K. Dantu, S. Rangwala, A. Sridharan, et al., "Networked sensing for structural health monitoring," in Proceedings of the 4th International Workshop on Structural Control, pp. 5766, Columbia University NY, 2004.

[48] J. P. Lynch, A. Sundararajan, K. Law, A. Kiremidjian, and E. Carryer, "Power-efficient data management for a wireless structural monitoring system," in Proceedings of the 4th International Workshop on Structural Health Monitoring, vol. 1, pp. 15-17, Citeseer, 2003.

[49] J. P. Lynch, A. Sundararajan, K. H. Law, A. S. Kiremidjian, and E. Carryer, "Embedding damage detection algorithms in a wireless sensing unit for operational power efficiency," Smart Materials and Structures, vol. 13, no. 4, p. 800, 2004.

[50] D. Salomon, Data compression: the complete reference. Springer Science \& Business Media, 2004. 
[51] S. Park, J.-J. Lee, C.-B. Yun, and D. J. Inman, “Electro-Mechanical Impedance-Based Wireless Structural Health Monitoring Using PCAData Compression and k-means Clustering Algorithms," Journal of Intelligent Material Systems and Structures, vol. 19, no. 4, pp. 509-520, 2008.

[52] E. Sazonov, K. Janoyan, and R. Jha, "Wireless intelligent sensor network for autonomous structural health monitoring," in Smart Structures and Materials, pp. 305-314, International Society for Optics and Photonics, 2004.

[53] R. Rajagopalan and P. K. Varshney, "Data aggregation techniques in sensor networks: A survey," 2006.

[54] S. Chatterjea and P. Havinga, "A Dynamic Data Aggregation Scheme for Wireless Sensor Networks," in ProRISC 2003, 14th Workshop on Circuits, Systems and Signal Processing, 2003.

[55] M. Thein and T. Thein, "An Energy Efficient Cluster-Head Selection for Wireless Sensor Networks," in Intelligent Systems, Modelling and Simulation (ISMS), 2010 International Conference on, pp. 287-291, Jan 2010.

[56] N. Aslam, W. Phillips, W. Robertson, and S. Sivakumar, "A multicriterion optimization technique for energy efficient cluster formation in wireless sensor networks," Information Fusion, vol. 12, no. 3, pp. 202-212, 2011.

[57] H. Sohn, "Effects of environmental and operational variability on structural health monitoring," Philosophical Transactions of the Royal Society A: Mathematical, Physical and Engineering Sciences, vol. 365, no. 1851, pp. 539-560, 2007. 
[58] J. Gao, J. Hu, G. Min, and L. Xu, "QoS analysis of medium access control in LR-WPANs under bursty error channels," Future Generation Computer Systems, vol. 26, no. 8, pp. 1426-1432, 2010.

[59] S. Wijetunge, U. Gunawardana, and R. Liyanapathirana, "Performance analysis of IEEE 802.15.4 MAC protocol for WSNs in burst error channels," in Communications and Information Technologies (ISCIT), 2011 11th International Symposium on, pp. 286-291, Oct 2011.

[60] B. Nefzi and Y.-Q. Song, "CoSenS: A collecting and sending burst scheme for performance improvement of IEEE 802.15.4," in Proceedings of the IEEE 35th Conference on Local Computer Networks (LCN), (Denver, CO, USA), pp. 172-175, 10-14 Oct 2010.

[61] J.-H. Lee, J.-K. Choi, and S.-J. Yoo, "Group Node Contention Algorithm for Avoiding Continuous Collisions in LR-WPAN," in Proceedings of the 9th IEEE International Conference on Computer and Information Technology (CIT), (Xiamen, China), pp. 69-74, Oct 2009.

[62] M.-Y. Cheng, Y.-B. Chen, H.-Y. Wei, and W. Seah, "Event-driven energy-harvesting wireless sensor network for structural health monitoring," in Proceedings of the IEEE 38th Conference on Local Computer Networks (LCN), (Sydney, Australia), pp. 364-372, 21-24 Oct 2013.

[63] M. Handy, M. Haase, and D. Timmermann, "Low energy adaptive clustering hierarchy with deterministic cluster-head selection," in Mobile and Wireless Communications Network, 2002. 4th International Workshop on, pp. 368-372, 2002.

[64] C. Li, M. Ye, G. Chen, and J. Wu, "An energy-efficient unequal clustering mechanism for wireless sensor networks," in Mobile Adhoc and Sensor Systems Conference, 2005. IEEE International Conference on, pp. 8-pp, IEEE, Nov 2005. 
[65] H. Huang and J. Wu, "A probabilistic clustering algorithm in wireless sensor networks," in IEEE Vehicular Technology Conference, vol. 62, p. 1796, IEEE; 1999, 2005.

[66] N. Shigei, H. Morishita, and H. Miyajima, "Energy efficient clustering communication based on number of neighbors for wireless sensor networks," in Proceedings of the International Multi Conference of Engineers and Computer Scientists, pp762-767, 2010.

[67] Y. Ma, Y. Guo, X. Tian, and M. Ghanem, "Distributed clusteringbased aggregation algorithm for spatial correlated sensor networks," Sensors Journal, IEEE, vol. 11, no. 3, pp. 641-648, 2011.

[68] R. Virrankoski and A. Savvidees, "TASC: topology adaptive spatial clustering for sensor networks," in Mobile Adhoc and Sensor Systems Conference, 2005. IEEE International Conference on, pp. 10-pp, IEEE, 2005.

[69] S. D. Muruganathan, D. C. Ma, R. I. Bhasin, and A. Fapojuwo, “A centralized energy-efficient routing protocol for wireless sensor networks," Communications Magazine, IEEE, vol. 43, no. 3, pp. S8-13, 2005.

[70] W. B. Heinzelman, A. P. Chandrakasan, and H. Balakrishnan, "An application-specific protocol architecture for wireless microsensor networks," Wireless Communications, IEEE Transactions on, vol. 1, no. 4, pp. 660-670, 2002.

[71] T. Kaur and J. Baek, "A strategic deployment and cluster-header selection for wireless sensor networks," Consumer Electronics, IEEE Transactions on, vol. 55, no. 4, pp. 1890-1897, 2009.

[72] S. Soro and W. B. Heinzelman, "Prolonging the lifetime of wireless sensor networks via unequal clustering," in Parallel and Distributed 
Processing Symposium, 2005. Proceedings. 19th IEEE International, pp. 8pp, IEEE, 2005.

[73] G. Anastasi, L. Lenzini, and E. Mingozzi, "HIPERLAN/1 MAC protocol: stability and performance analysis," Selected Areas in Communications, IEEE Journal on, vol. 18, pp. 1787-1798, Sept 2000.

[74] Y. Tay, K. Jamieson, and H. Balakrishnan, "Collision-minimizing CSMA and its applications to wireless sensor networks," Selected Areas in Communications, IEEE Journal on, vol. 22, pp. 1048-1057, Aug 2004.

[75] C. Gezer, C. Buratti, and R. Verdone, "Capture effect in IEEE 802.15.4 networks: Modelling and experimentation," in Proceedings of the 5th IEEE International Symposium on Wireless Pervasive Computing (ISWPC), (Modena, Italy), pp. 204-209, 5-7 May 2010.

[76] J. Lu and K. Whitehouse, "Flash Flooding: Exploiting the Capture Effect for Rapid Flooding in Wireless Sensor Networks," in Proceedings of the 28th IEEE Conference on Computer Communications (INFOCOM), (Rio de Janeiro, Brazil), pp. 2491-2499, 19-25 April 2009.

[77] A. Deraemaeker, E. Reynders, G. D. Roeck, and J. Kullaa, “Vibrationbased structural health monitoring using output-only measurements under changing environment," Mechanical Systems and Signal Processing, vol. 22, no. 1, pp. 34-56, 2008.

[78] R. RUOTOLO and C. SURACE, "Using SVD to detect damage in structures with different operational conditions," Journal of Sound and Vibration, vol. 226, no. 3, pp. 425-439, 1999.

[79] M. Lemistre and D. Balageas, "Structural health monitoring system based on diffracted Lamb wave analysis by multiresolution processing," Smart Materials and Structures, vol. 10, no. 3, p. 504, 2001. 
[80] E. Casilari, J. M. Cano-García, and G. Campos-Garrido, "Modeling of Current Consumption in 802.15. 4/ZigBee Sensor Motes," Sensors, vol. 10, no. 6, pp. 5443-5468, 2010.

[81] C. Noda, S. Prabh, M. Alves, and T. Voigt, "On packet size and error correction optimisations in low-power wireless networks," in Proceedings of the 10th Annual IEEE Communications Society Conference on Sensor, Mesh and Ad Hoc Communications and Networks (SECON), (New Orleans, LA, USA), pp. 212-220, 24-27 June 2013. 